\title{
Rigorous Investigation of AlGaN/GaN Heterostructure Surface Treatments with Si Thin Films
}

\author{
by
}

Artur Kochermin

A Thesis submitted to

the Faculty of Graduate Studies and Research

in partial fulfilment of

the requirements for the degree of

\begin{abstract}
Master of Applied Science in Electrical Engineering
Ottawa-Carleton Institute for Electrical and Computer Engineering

Department of Electronics, Carleton University

Ottawa, Ontario, Canada

January 2011
\end{abstract}

Copyright (C)

2011 - Artur Kochermin 


$\begin{array}{ll}\begin{array}{l}\text { Library and Archives } \\ \text { Canada }\end{array} & \begin{array}{l}\text { Bibliotheque et } \\ \text { Archives Canada }\end{array} \\ \begin{array}{l}\text { Published Heritage } \\ \text { Branch }\end{array} & \begin{array}{l}\text { Direction du } \\ \text { Patrimoine de l'édition }\end{array} \\ 395 \text { Wellington Street } & 395, \text { rue Wellington } \\ \text { Ottawa ON K1A ON4 } & \text { Ottawa ON K1A 0N4 } \\ \text { Canada } & \text { Canada }\end{array}$

Your file Votre référence
ISBN: $978-0-494-79532-3$
Our file Notre référence
ISBN: $978-0-494-79532-3$

NOTICE:

AVIS:

The author has granted a nonexclusive license allowing Library and Archives Canada to reproduce, publish, archive, preserve, conserve, communicate to the public by telecommunication or on the Internet, loan, distribute and sell theses worldwide, for commercial or noncommercial purposes, in microform, paper, electronic and/or any other formats.

The author retains copyright ownership and moral rights in this thesis. Neither the thesis nor substantial extracts from it may be printed or otherwise reproduced without the author's permission.

L'auteur a accordé une licence non exclusive permettant à la Bibliothèque et Archives Canada de reproduire, publier, archiver, sauvegarder, conserver, transmettre au public par télécommunication ou par l'Internet, prêter, distribuer et vendre des thèses partout dans le monde, à des fins commerciales ou autres, sur support microforme, papier, électronique et/ou autres formats.

L'auteur conserve la propriété du droit d'auteur et des droits moraux qui protège cette thèse. $\mathrm{Ni}$ la thèse ni des extraits substantiels de celle-ci ne doivent être imprimés ou autrement reproduits sans son autorisation.

In compliance with the Canadian Privacy Act some supporting forms may have been removed from this thesis.

While these forms may be included in the document page count, their removal does not represent any loss of content from the thesis.
Conformément à la loi canadienne sur la protection de la vie privée, quelques formulaires secondaires ont été enlevés de cette thèse.

Bien que ces formulaires aient inclus dans la pagination, il n'y aura aucun contenu manquant.

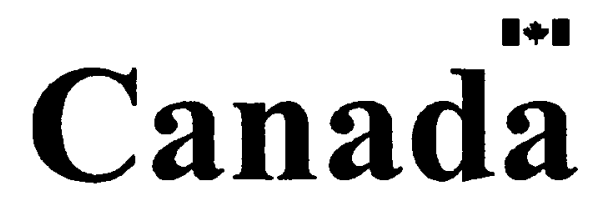




\section{Abstract}

Wide bandgap AlGaN/GaN High Electron Mobility Transistors (HEMTs) are currently the subject of intensive research and have already demonstrated an unprecedented performance in high power, high frequency and high temperature areas of applications. In order to improve ohmic contacts and to address the performance degradation due to surface states on this material, several research groups have used Si-containing films on the semiconductor surface.

In this study a rigorous investigation of the "treated" and "coated" AlGaN/GaN surfaces with two different Si-containing thin films was carried out. "Treatment" is defined as the deposition of Si-containing thin film, encapsulation with $\mathrm{SiO}_{2}$, Rapid Thermal Annealing (RTA) and the films removal with a wet etch. "Coating" is defined as the deposition of $\mathrm{Si}$ film on the $\mathrm{AlGaN} / \mathrm{GaN}$ surface left in place for all further processing. Treated, coated and control samples were examined with Secondary Ion Mass Spectroscopy (SIMS), X-ray Photo-electron Spectroscopy (XPS), Hall and contact angle measurements in order to establish the physical mechanism responsible for the electrical changes on the Si-treated surfaces. Previous workers have attributed the improvement in the electrical properties to deep diffusion of Si species into the GaN or AlGaN epilayers, as indicated by SIMS profiling. In this work, it is demonstrated that this detection of Si was due to a SIMS profiling artifact.

A remnant $\mathrm{SiN}_{x}$ film with the approximate thickness between 1.0 and $2.0 \mathrm{~nm}$ was identified by SIMS and XPS on the Si-treated AlGaN surfaces after the Si film was 
removed with an aggressive $\mathrm{HF}: \mathrm{HNO}_{3}$ wet etch. The water contact angle measurement has revealed the similarity between Si-coated and Si-treated samples, and the results were in sharp contrast to the measurements on the control samples.

The Si treatments and coatings were found to pre-passivate the AlGaN surface, significantly improving the Two-Dimensional Electron Gas (2DEG) sheet resistances and current densities before silicon nitride passivation. The best ohmic contact resistance values were not found to be affected significantly by the treatments or coatings and remained in the range of $0.5-0.6 \Omega-\mathrm{mm}\left(\rho_{c} \approx 6.3 \times 10^{-6}-9.0 \times 10^{-6} \Omega-\mathrm{cm}^{2}\right)$. However, Si treatments and coatings were found to significantly improve the ohmic contact resistance values in adverse or poorly optimized processing conditions. Both the treatments and the coatings have resulted in higher leakage currents and more negative threshold voltages in fabricated devices.

It was concluded that the solid state diffusion was not responsible for the electrical enhancements in fabricated devices. The electrical changes observed in this study were attributed to the remnant $\mathrm{SiN}_{x}$ film on Si-treated and Si film on Si-coated AlGaN surfaces. 


\section{Acknowledgments}

I would like to express my sincere gratitude to everyone who aided and supported me in my research. I am very grateful for the opportunity to conduct my research on the exciting and fairly new Gallium Nitride technology and for the opportunity to use both Carleton University's and National Research Council's Institute for Microstructural Science (NRC-IMS) fabrication facilities.

I am very thankful to my graduate supervisors Garry Tarr (Carleton University) and Jennifer Bardwell (NRC-IMS) for their guidance, extensive training and support. Their dedication and involvement in my work, as well as readiness to address any questions and concerns, were extremely valuable to me. I would also like to thank Tim Lester (NRC-IMS) and Margaret Buchanan (NRC-IMS) for laboratory training, helpful discussions and support. I thank Dan Roth, Hue Tran, Simona Moisa, Zhenhua He, Rongzhu Wang, John Hulse, Jiayun Zhou, Rob Vandusen, Angela Burns, Carol Adams, Rick Adams and others who helped me with the fabrication work at NRC-IMS and Carleton Microfabrication laboratories.

Special thanks go to my friends and colleague-students, in particular at Carleton and NRC. My coffee breaks just would not be the same without you.

Finally, I thank my wife Vera for the encouragement, support and understanding, and to my little son Albert for brightening my days with his smile. 


\section{Table of Contents}

$\begin{array}{ll}\text { Abstract } & \text { iii }\end{array}$

$\begin{array}{lll}\text { Acknowledgments } & \text { v }\end{array}$

Table of Contents viii

List of Tables $\quad$ x

List of Figures $\quad$ xiv

Nomenclature $\quad$ XV

1 INTRODUCTION 1

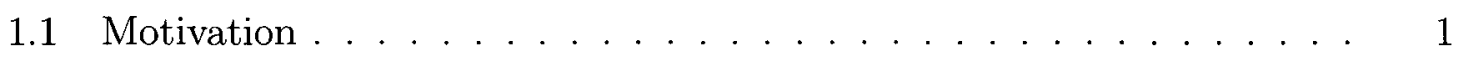

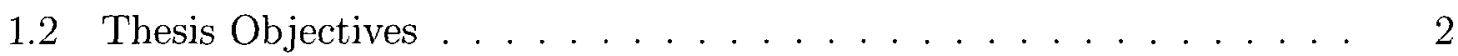

1.3 Thesis Organization . . . . . . . . . . . . . . 2

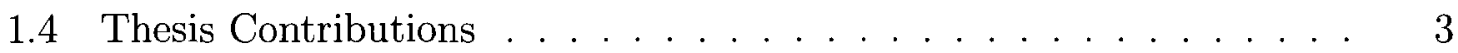

2 GAN TECHNOLOGY OVERVIEW 4

2.1 GaN Materials and Devices ................. 4

2.1.1 Material properties and growth ............ 4

2.1.2 Doping of GaN materials . . . . . . . . . . . . . 8

2.1.3 High electron mobility transistors .............. 11 
2.1.4 Current collapse and silicon nitride passivation . . . . . . . 21

2.1.5 Metal contacts to semiconductor materials . . . . . . . 22

2.1.6 Ohmic and Schottky contacts to GaN and AlGaN/GaN . . . 31

2.1.7 Surface preparation, cleaning and chemical analysis . . . . . 33

2.2 Literature Review on GaN and AlGaN/GaN Surface Treatment with

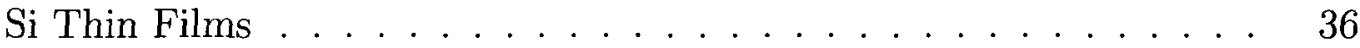

\section{DESIGN AND FABRICATION}

3.1 Process Flow Considerations and Starting Material _. . . . . . . 45

3.2 Batch-1 Process Flow Design and Measurements . . . . . . . . . . . 47

3.3 Batch-2 Process Flow Design and Measurements . . . . . . . . . . 51

3.4 Batch-3 Process Flow Design and Measurements . . . . . . . . . . . . 54

4 RESULTS

4.1 Introduction . . . . . . . . . . . . . . . . . 57

4.2 2DEG Sheet Resistance, Electron Density and Mobility by Hall . . . 58

$4.2 .1 \quad$ Batch $-1 \ldots \ldots \ldots \ldots \ldots \ldots \ldots$

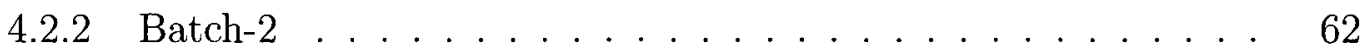

4.3 Ohmic Contacts and 2DEG Sheet Resistance from c-TLM and TLM Structures .......................... 63

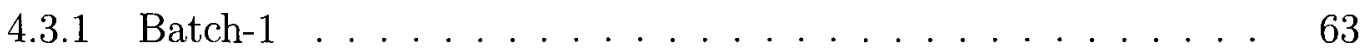

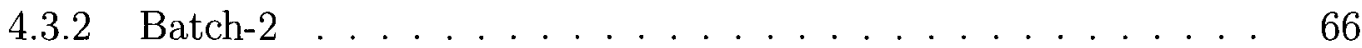

4.3 .3 Batch-3 . . . . . . . . . . . . . . . . . 69

4.4 Ohmic Contact Surface Morphology by Visual Inspection and Surface Profiling . . . . . . . . . . . . . . . . . . . 74

4.5 HEMT DC and Pulsed I-V Curves/ Current Collapse . . . . . . . . 76

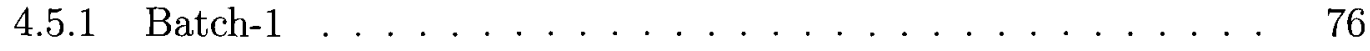

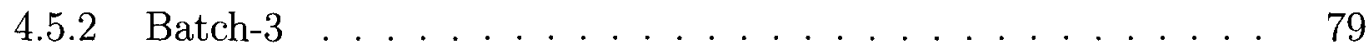


4.6 HEMTs Threshold Voltage and Transconductance . . . . . . . . . . 83

4.6 .1 Batch-3 ................... 83

4.7 HEMT Gate Schottky Junction I-V Curves . . . . . . . . . . . . . . . 87

4.7 .1 Batch-1 ...................... 87

4.7 .2 Batch-3 .................... 88

4.8 Drain-to-Source Breakdown Voltage . . . . . . . . . . . . . . . . 89

4.8.1 Batch-1 .................... 89

4.9 SIMS AlGaN/GaN Depth Profiling . . . . . . . . . . . . . . . 90

4.10 AlGaN Surface Chemical Composition and Film Thickness by XPS and AR-XPS ......................... 93

4.11 AlGaN Surface Water Contact Angle . . . . . . . . . . . . . . . 100

5 DISCUSSION 102

6 CONCLUSIONS AND FUTURE WORK 108

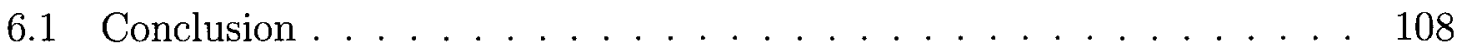

6.2 Future Work . . . . . . . . . . . . . . . . . . 109

$\begin{array}{ll}\text { List of References } & 117\end{array}$

Appendix A GaN IC FABRICATION PROCESS FLOW 118

A.1 NRC's standard GaN IC Fabrication Process Flow (some information is intentionally omitted) . . . . . . . . . . . . . . . 118

A.2 Batch-1 Fabrication Process Flow . . . . . . . . . . . . . . 120

A.3 Batch-2 Fabrication Process Flow . . . . . . . . . . . . . . . . . . . 122

A.4 Batch-3 Fabrication Process Flow . . . . . . . . . . . . . . . . 123 


\section{List of Tables}

2.1 Comparison table of several most common semiconductor bulk prop-

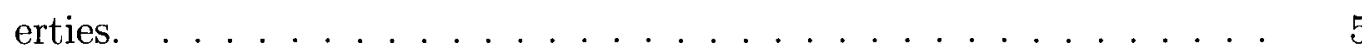

2.2 Work functions and resistivity of several metals suitable for Schottky

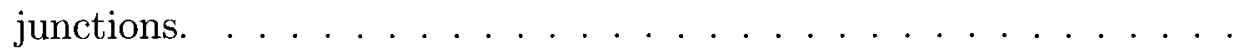

3.1 Batch-1 samples and the details on corresponding critical process steps and material measurements. The number in brackets indicates the Hall measurement label. . . . . . . . . . . . . . . . . . . . . . . . . .

3.2 Batch-2 samples and the details on corresponding critical process steps and material measurements. The number in brackets indicates the Hall measurement label.

3.3 Batch-3 samples and the details on corresponding critical process steps and material measurements. . . . . . . . . . . . . . . .

4.1 Batch-2 2DEG sheet resistance, electron mobility and density before the deposition of the a-Si thin film and after the $\mathrm{HF}: \mathrm{HNO}_{3}$ wet etch.

4.2 Batch-1 ohmic contact and 2DEG sheet resistances from a four point probe measurement of c-TLM and TLM structures. Averages of two to four measurements were taken for each sample. 1ME TLM results combine the measurements of ohmic-probeable as well as of 100x64 and

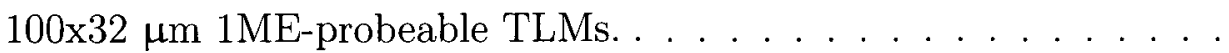


4.3 Batch-2 ohmic contact, 2DEG sheet resistances and current density over $2 \mu \mathrm{m}$ c-TLM gap from a four point probe measurement of c-TLM and TLM structures during RTA annealing. Averages of two to four measurements were taken for each sample. '*' denotes non-linearity in TLM data. . . . . . . . . . . . . . . . . . . .

4.4 Batch-3 ohmic contact, 2DEG sheet resistances and current density over $2 \mu \mathrm{m}$ c-TLM gap from a four point probe measurement of c-TLM and TLM structures during RTA annealing. Averages of two to four measurements were taken for each sample. '*' denotes non-linearity in TLM data. . . . . . . . . . . . . . . . . . . . . . .

4.5 Batch-3 ohmic contact, 2DEG sheet resistances and current density over $2 \mu \mathrm{m}$ c-TLM gap from a four point probe measurement of cTLM and TLM structures. Averages of two to six measurements were taken for each sample. 1ME TLM results combine the measurements of ohmic-probeable as well as of 100x64 and 100x32 $\mu \mathrm{m}$ 1ME-probeable TLMs. . . . . . . . . . . . . . . . . .

4.6 Batch-3 transconductance and threshold voltage summary. . . . . . . 86

4.7 Batch-1 gate Schottky junction I-V summary after passivation and $1 \mathrm{ME}$ dep. processing steps. . . . . . . . . . . . . . . . .

4.8 Batch-3 gate Schottky junction I-V summary after passivation and $1 \mathrm{ME}$ dep. processing steps. . . . . . . . . . . . . . . . 


\section{List of Figures}

2.1 Electron drift velocity at $300 \mathrm{~K}$ in $\mathrm{GaN}, \mathrm{SiC}, \mathrm{Si}$ and GaAs computed using the Monte Carlo technique. . . . . . . . . . . . . 5

2.2 Bandgap energy at $300 \mathrm{~K}$ as a function of lattice constant of III-N and other semiconductors. . . . . . . . . . . . . . . . . 6

2.3 Hexagonal crystal system and different polarities (Ga- and N-faced) of wurtzite GaN. . . . . . . . . . . . . . . . . . . . . 7

$2.4 \mathrm{AlGaN} / \mathrm{GaN}$ HEMT Structure. . . . . . . . . . . . . . . 12

2.5 Polarization and induced charges in crystal structure and epilayers in GaN/AlGaN/GaN Ga- and N-faced HEMTs. . . . . . . . . . . . . . . 14

2.6 Conduction band diagram of AlGaN/GaN HEMT. . . . . . . . . . .

2.7 Polarization induced bound interface sheet densities and 2DEG density as a function of $\mathrm{Al}$ mole fraction for a $\mathrm{Ga}$-face $\mathrm{AlGaN} / \mathrm{GaN}$ heterostructure $(30 / 2,000 \mathrm{~nm}) \ldots \ldots \ldots 17$

$2.82 \mathrm{DEG}$ density dependence on AlGaN layer thickness. . . . . . . . . . 18

2.9 Conduction band diagram of $\mathrm{AlGaN} / \mathrm{GaN}$ and $\mathrm{AlGaN} / \mathrm{AlN} / \mathrm{GaN}$ heterostructures. Dipole in AlN increases the effective $\Delta E^{C}\left(\Delta E_{C}\right.$ in the figure). . . . . . . . . . . . . . . . . . .

2.10 Calculated electron velocity vs electric field for AlGaNGaN HEMT structures with Al mole fractions of $0.15,0.2$ and $0.3 . . . . . .$. 
2.11 DC and pulsed I-V characteristics of an unpassivated AlGaN/GaN HEMT on SiC substrate. Current collapse (DC to RF dispersion) could be observed in the pulsed mode. . . . . . . . . . . 20

$2.12 \mathrm{I}-\mathrm{V}$ characteristic curves for ohmic and Schottky contacts. . . . . . 23

2.13 Schottky contact formation. . . . . . . . . . . . . . . 24

2.14 Ohmic contact formation. . . . . . . . . . . . . . 26

2.15 Tunnelling ohmic contact band diagram. . . . . . . . . . . 27

2.16 TLM test structure. . . . . . . . . . . . . . . . . . . . 29

2.17 Resistance measured between adjacent TLM structure contacts versus their spacing. . . . . . . . . . . . . . . . . 30

2.18 c-TLM structure. . . . . . . . . . . . . . . . . . . . 31

2.19 SIMS ${ }^{28}$ Si profiles taken from a Si doped and non-doped samples. . . 39

2.20 Schematic model of the atomic arrangement at the SiN/AlGaN interface. 43

3.1 Lithographic photo-mask. TLM, c-TLM and HEMT structures are probeable after ohmic metal anneal. . . . . . . . . . . . .

4.1 Batch-1 evolution of the 2DEG sheet resistance $R_{s}$ as a function of processing steps. The spread associated with each data point is $\sim 5$ $10 \mathrm{Ohm} / \mathrm{sq}$. Step 1 and 2: before and after the deposition of the thin films. Steps 3-14: RTA anneals. Step 15: after the HF: $\mathrm{HNO}_{3}$ wet etch.

4.2 Batch-1 evolution of the 2DEG electron mobility $\mu$ as a function of processing steps. Step 1 and 2: before and after the deposition of the thin films. Steps 3-14: RTA anneals. Step 15: after the HF: $\mathrm{HNO}_{3}$ wet

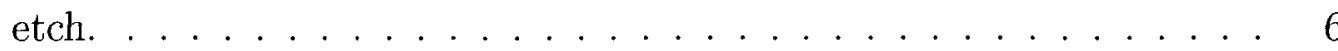

4.3 Batch-1 evolution of the 2DEG electron density $n_{s}$ as a function of processing steps. Step 1 and 2: before and after the deposition of the thin films. Steps 3-14: RTA anneals. Step 15: after the HF: $\mathrm{HNO}_{3}$ wet

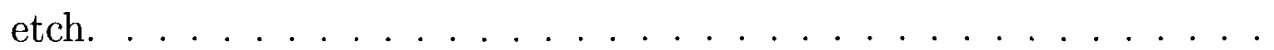


4.4 Batch-1 ohmic contact and 2DEG sheet resistances after passivation and 1ME deposition steps. . . . . . . . . . . . . . . 65

4.5 Batch-2 2DEG sheet resistance values $\left(\mathrm{R}_{s}\right)$ after ohmic contact RTA anneals. $835^{\circ} \mathrm{C}$ results were used for Si-treated samples. $840^{\circ} \mathrm{C}$ results were used for ${ }^{\dagger} \mathrm{B} 1, \mathrm{~A} 1$ and Si-coated samples. Pre-passivating effects of Si treatments and coatings can be clearly seen. . . . . . . . . .

4.6 Batch-3 final 2DEG sheet resistance values $\left(\mathrm{R}_{s}\right)$ after ohmic contact RTA anneals. Pre-passivating effects of Si treatments and coatings can be clearly seen. . . . . . . . . . . . . . . . . . .

4.7 Batch-3 ohmic contact and 2DEG sheet resistances after passivation and $1 \mathrm{ME}$ deposition steps. . . . . . . . . . . . . . . .

4.8 Ohmic metal morphology problems and their effect on 1ME overlayer on samples $\mathrm{C} 1$ in Batch-2 and $\mathrm{C} 1$ in Batch-3. . . . . . . . . . . . . 75

4.9 Batch-1 DC and pulsed I-V measurements of unpassivated and passivated HEMTs on samples ${ }^{\dagger} \mathrm{A} 1$ and B1. . . . . . . . . . . .

4.10 Batch-1 DC and pulsed I-V measurements of unpassivated and passi-

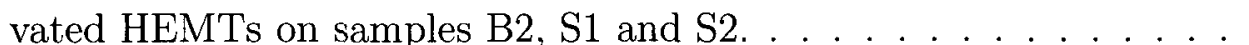

4.11 Batch-1 DC and pulsed I-V measurements of unpassivated and passivated HEMTs on samples $\mathrm{O} 1$ and $\mathrm{O} 2 \ldots \ldots \ldots$

4.12 Batch-3 DC and pulsed I-V measurements of unpassivated and passivated HEMTs on samples ${ }^{\dagger} \mathrm{B} 1$ and ${ }^{\dagger} \mathrm{B} 2 \ldots \ldots . \ldots . \ldots 80 . \ldots$

4.13 Batch-3 DC and pulsed I-V measurements of unpassivated and passivated HEMTs on samples S1 and S2. .............

4.14 Batch-3 DC and pulsed I-V measurements of unpassivated and passivated HEMTs on samples $\mathrm{C} 1$ and $\mathrm{C} 2 \ldots \ldots \ldots$

4.15 Batch-3 threshold voltage and reverse bias leakage current measurement on samples ${ }^{\dagger} \mathrm{B} 1$ and $\mathrm{S} 1 . \ldots \ldots . \ldots . \ldots 84$ 
4.16 Batch-3 threshold voltage and reverse bias leakage current measurement on samples $\mathrm{C} 1$ and $\mathrm{C} 2$. . . . . . . . . . . . . . 85

4.17 Batch-3 HEMTs transconductance measurements on samples ${ }^{\dagger} \mathrm{B} 1$ and

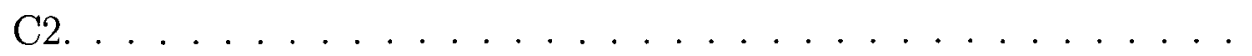

4.18 Batch-1 gate Schottky junction I-V curves after the passivation and 1ME deposition steps. . . . . . . . . . . . . . .

4.19 Batch-1 drain-to-source breakdown voltage measurements. Gate voltage $\left(V_{g}\right)$ was set to $-5 \mathrm{~V}$. . . . . . . . . . . . . 89

4.20 Batch-2 SIMS ${ }^{27} \mathrm{Al}$ profile of the control sample ${ }^{\dagger} \mathrm{B} 1 . \quad \ldots . . . . . .991$

4.21 Batch-2 SIMS ${ }^{28} \mathrm{Si}$ profile of the control sample as well as Si-treated and Si-coated reference samples. . . . . . . . . . . . . . . . . 92

4.22 Batch-2 R2 XPS spectra before, after 10 and $30 \mathrm{~min}$ of Ar sputtering.

4.23 Batch-3 S2 XPS spectra before and after processing steps leading to ohmic contact metal deposition. . . . . . . . . . . . . . . . 95

4.24 The location of several Si $2 p$ peaks from peak fitting performed by Matsuoka et al. ................... 96

4.25 Batch-3 S2 AR-XPS spectra at $30^{\circ}, 45^{\circ}$ and $77.5^{\circ}$ take off angle. . . . 97

4.26 The fit of the AR-XPS equation to the experimental data using Si to Ga sensitivity ratio parameter $\beta$ of 0.32 , photoelectron attenuation length $\lambda$ of $3 \mathrm{~nm}$ and the film thickness $d$ of $2.45 \mathrm{~nm}$. Theoretical curves with the values of $d=1.95 \mathrm{~nm}$ and $d=2.95 \mathrm{~nm}$ are also shown for comparison. ....................... . . . . . . . .

4.27 Combined plot of XPS spectra for several samples from Batch-2 and

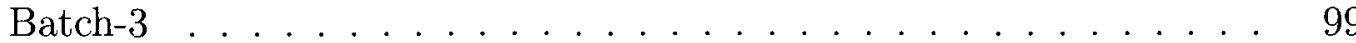

4.28 Batch-3 water contact angle measurement result. . . . . . . . . . . . 101 


\section{Nomenclature}

2-DEG Two-Dimensional Electron Gas

AR-XPS Angular Resolved X-ray Photo-electron Spectroscopy

BOE Buffered Oxide Etchant

c-TLM Circular Transmission Line Model

DI Deionized (Water)

FE $\quad$ Field Emission

FWHM Full Width at Half Maximum

IPA Isopropyl Alcohol

HEMT High Electron Mobility Transistor

HFET Heterostructure Field Effect Transistor

HMDS Hexamethyldisilazane, Adhesion Promoter

ICP Inductively Coupled Plasma

MBE Molecular Beam Epitaxy

MOCVD Metal-Organic Chemical Vapour Deposition

NRC National Research Council

PECVD Plasma-Enhanced Chemical Vapour Deposition

RTA Rapid Thermal Annealing

SBH Schottky Barrier Height

SIMS Secondary Ion Mass Spectroscopy

TE Thermionic Emission

TFE Thermionic Field Emission

TLM Transmission Line Model 
WZ

XPS

$\mathrm{ZB}$
Wurtzite, Crystal Structure

X-ray Photo-electron Spectroscopy

Zincblende, Crystal Structure 


\section{Chapter 1}

\section{INTRODUCTION}

\subsection{Motivation}

The wide bandgap GaN semiconductor material has generated an enormous amount of interest in recent years due to its unique properties and unchallenged performance in high power, high temperature and high frequency applications. Due to the wide bandgap, high electron mobility, high breakdown electric field and unusually strong polarization properties, AlGaN/GaN based High Electron Mobility Transistors (HEMTs) have reached power densities of $30-40 \mathrm{~W} / \mathrm{mm}$, more than one order of magnitude higher than GaAs. At the same time, unity gain frequency $\mathrm{f}_{T}$ and maximum oscillation frequency $\mathrm{f}_{\max }$ of about $200 \mathrm{GHz}$ have been achieved, extending the application of GaN devices to millimetre wave and beyond [1].

Making good ohmic contacts to $\mathrm{GaN}$ and $\mathrm{AlGaN} / \mathrm{GaN}$ heterostructures remains a major challenge to this day. Research aimed at improvements to ohmic contacts and/or sheet carrier densities in $\mathrm{AlGaN} / \mathrm{GaN}$ heterostructures, as well as better insights into the physical mechanisms underlying these enhancements, would aid substantially to the development of superior GaN electronic devices.

One proposed method to improve ohmic contacts to GaN or AlGaN/GaN material relies on the apparent doping of the surface with a thin Si-containing film using high 
temperature treatments (referred to as $\mathrm{Si}$ "treatment"). Evidence for this supposed solid state diffusion of Si was limited to Secondary Ion Mass Spectroscopy (SIMS) profiles obtained in previous work, which is reviewed in Section 2.2. Appropriate control samples for SIMS measurements were not included or analyzed. Thus, previously reported Si concentration depth profiling results obtained by SIMS were likely to contain a primary ion forward scattering artifact, making the Si diffusion appear deeper. The mechanisms by which the process steps involved in Si treatments could improve the electrical performance of $\mathrm{AlGaN} / \mathrm{GaN}$ heterostructures were not fully investigated and properly identified. The necessity of Si diffusion for better ohmic contacts is questionable and was not addressed by the previous research. In addition, carrier densities after Si wet etch and subsequent HEMT fabrication were not previously reported.

\subsection{Thesis Objectives}

The primary objective of this work was to identify the main mechanism behind the improvements to ohmic contacts and carrier sheet densities in AlGaN/GaN material achieved by $\mathrm{Si}$ treatments. The second objective of this work was to investigate AlGaN/GaN structures on which the Si film was intentionally left in place for all further processing (referred to as $\mathrm{Si}$-"coated"), and compare them to the Si-treated material.

\subsection{Thesis Organization}

An overview of GaN materials and devices, as well as a review of previous work on the use of $\mathrm{Si}$ on $\mathrm{GaN}$ and AlGaN surfaces are given in Chapter 2. Process flow considerations and design as well as the performed electrical and chemical composition 
measurements are given in Chapter 3. Chapter 4 briefly explains and presents each measurement result. Chapter 5 provides discussion of the results. Finally, thesis summary and areas for future work are given in Chapter 6 .

\subsection{Thesis Contributions}

As a result of this research, it was established that deep diffusion of Si into AlGaN layer is not responsible for the enhancement to the electrical performance of Al$\mathrm{GaN} / \mathrm{GaN}$ devices. A remnant $\mathrm{SiN}_{x}$ film with the approximate thickness between 1.0 and $2.0 \mathrm{~nm}$, produced by the solid state reaction, remained on AlGaN surface even after the aggressive $\mathrm{HF}: \mathrm{HNO}_{3}$ wet etch of $\mathrm{Si}$ and $\mathrm{SiO}_{2}$. The film was analyzed by X-ray Photo-electron Spectroscopy (XPS), SIMS and water contact angle measurements. The forward scattering of Si by the primary ion beam artifact during SIMS profiling was demonstrated. The remnant $\mathrm{SiN}_{x}$ and an intentionally left thin film of $\mathrm{Si}$ on $\mathrm{AlGaN}$ surface were shown to pre-passivate the AlGaN material. The treatment was also found to yield better ohmic contact resistance values in adverse or poorly optimized processing conditions. 


\section{Chapter 2}

\section{GAN TECHNOLOGY OVERVIEW}

\subsection{GaN Materials and Devices}

\subsubsection{Material properties and growth}

$\mathrm{GaN}$ is a direct wide bandgap semiconductor material with bandgap energy of $3.39 \mathrm{eV}$ at room temperature. The compound is very hard and chemically stable, with the melting point exceeding $2,500^{\circ} \mathrm{C}$. However, GaN surface undergoes decomposition at temperatures of about $1,000^{\circ} \mathrm{C}$ at a pressure of one atmosphere due to nitrogen out diffusion [2].

The wide bandgap allows the material to stay extrinsic for a wider range of temperatures, thus making $\mathrm{GaN}$ an ideal candidate for high temperature operation. Operating temperatures of several hundred degrees Celsius have been previously reported for GaN devices and circuits [3]. The critical breakdown electric field in semiconductors scales roughly with the square of the bandgap energy and is estimated to be in excess of $4 \mathrm{MV} / \mathrm{cm}$ for $\mathrm{GaN}$, compared with $0.2 \mathrm{MV} / \mathrm{cm}$ and $0.4 \mathrm{MV} / \mathrm{cm}$ for $\mathrm{Si}$ and GaAs respectively, making the material very suitable for high voltage operation [4]. Additionally, an adequate electron mobility and a superior saturation velocity make GaN very suitable for high frequency operation (Fig. 2.1). 


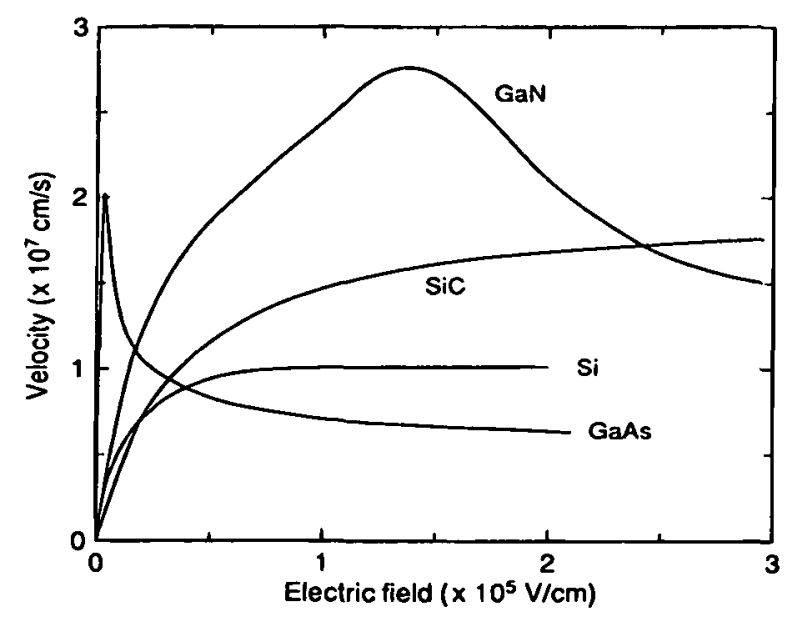

Figure 2.1: Electron drift velocity at $300 \mathrm{~K}$ in $\mathrm{GaN}, \mathrm{SiC}, \mathrm{Si}$ and GaAs computed using the Monte Carlo technique [4].

Table 2.1 compares measured bulk GaN properties with other semiconductors. GaN properties do not stand out significantly in comparison to other wide bandgap semiconductors such as $\mathrm{SiC}$ or diamond. Additionally, hole mobility in the material is exceptionally low, about $10 \mathrm{~cm}^{2} /(\mathrm{Vs})[2]$, although the material allows for $\mathrm{p}-\mathrm{n}$ junctions [4]. The main advantage of GaN comes from the material's support for ternary and quaternary compounds and HEMT structures [5]:

\begin{tabular}{|l||c|c|c|c|c|c|}
\hline Material & GaN & Si & GaAs & 3H-SiC & 4H-SiC & Diamond \\
\hline \hline Bandgap (eV) & 3.39 & 1.12 & 1.42 & 2.2 & 3.2 & 5.45 \\
\hline Bandgap Type & Direct & Indirect & Direct & Indirect & Indirect & Indirect \\
\hline Electron Mobility $\left(\mathrm{cm}^{2} /(\mathrm{Vs})\right)$ & 1,300 & 1,350 & 8,500 & 900 & 800 & 1,900 \\
\hline Saturation Velocity $\left(10^{7} \mathrm{~cm} / \mathrm{s}\right)$ & $2.5-3$ & 1 & 1 & 2 & 2 & 2.7 \\
\hline Critical Breakdown Field $(M V / \mathrm{cm})$ & 4 & $0.25-0.3$ & 0.4 & 1.2 & 3 & 5.6 \\
\hline Thermal Conductivity $(W / \mathrm{cm} \cdot \mathrm{K})$ & 1.3 & 1.5 & 0.43 & 4.9 & 4.9 & 20 \\
\hline Dielectric Constant $\left(\varepsilon_{r}\right)$ & 9.5 & 11.8 & 13.9 & 9.6 & 9.7 & 5.5 \\
\hline HEMT structures & yes & no & yes & no & no & no \\
\hline
\end{tabular}

Table 2.1: Comparison table of several most common semiconductor bulk properties [1].

GaN can form alloys with AlN and InN to form ternary and quaternary compounds which span a continuous range of direct bandgap energies from $0.8 \mathrm{eV}$ for InN to $6.2 \mathrm{eV}$ 
for AlN (Fig. 2.2). This allows III-N material system to cover a very broad range of energies and thus emission wavelengths from the infra-red to the deep ultraviolet are unchallenged by any other available material today [4]. The large range of available bandgaps and support for heterostructures in III-V materials also allows for bandgap engineering.

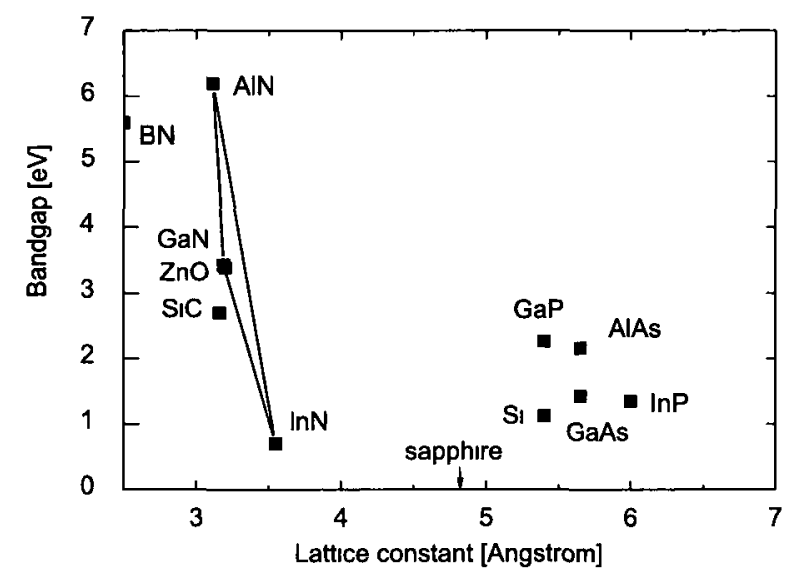

Figure 2.2: Bandgap energy at $300 \mathrm{~K}$ as a function of lattice constant of III-N and other semiconductors [5].

GaN, AlN and InN form a Wurtzite (WZ) crystal structure at room temperature (also referred to as $\alpha$-phase). Each element forms a tetrahedral covalent bond with its neighbouring atoms. The Zincblende (ZB) structure can also be found for GaN and InN in thin films. AlN is metastable and has an indirect bandgap in this crystal form. Its growth is also more complicated $[5,6]$.

WZ crystal structure is a member of hexagonal crystal system (Fig 2.3(a)). The crystal lattice structure of GaN can be described by the length of the hexagonal edge a, the height $\mathbf{c}$ of the prism, and the microscopic dimensionless parameter $\mathbf{u}$, which is defined as the length of the bond parallel to the $\mathbf{c}$ axis in units of $\mathbf{c}$. These parameters for AlN, GaN, and InN can be found in Ambacher et al. [2].

Four Bravais-Miller indices are used to describe directions and planes in hexagonal crystal lattices. WZ structure has polar axes (lack of inversion symmetry). In 
particular, the bonds in the $\langle 0001\rangle$ direction for wurtzite are all faced by nitrogen in the same direction and by the cation in the opposite direction. Both bulk and surface properties can depend significantly on whether the surface is faced by nitrogen or metal atoms. The most common growth direction of hexagonal GaN is normal to the $\{0001\}$ basal plane, where the atoms are arranged in bilayers consisting of two closely spaced hexagonal layers, one with cations and the other with anions, so that the bilayers have polar faces. Thus, in the case of GaN a basal surface should be either Ga- or $\mathrm{N}$-faced. By Ga-faced it is meant that $\mathrm{Ga}$ is on the top position of the $\{0001\}$ bilayer, corresponding to [0001] polarity (Fig. 2.3(b)). Ga-faced does not mean Ga-terminated; termination should only be used to describe a surface property. A Ga-face surface might be N-terminated if it is covered with nitrogen atoms, but without flipping the crystal it will never be $\mathrm{N}$-faced. It is, however, important to note that the (0001) and (0001) surfaces of GaN are inequivalent (by convention, the [0001] direction is given by a vector pointing from a $\mathrm{Ga}$ atom to a nearest-neighbour $\mathrm{N}$ atom) [2].

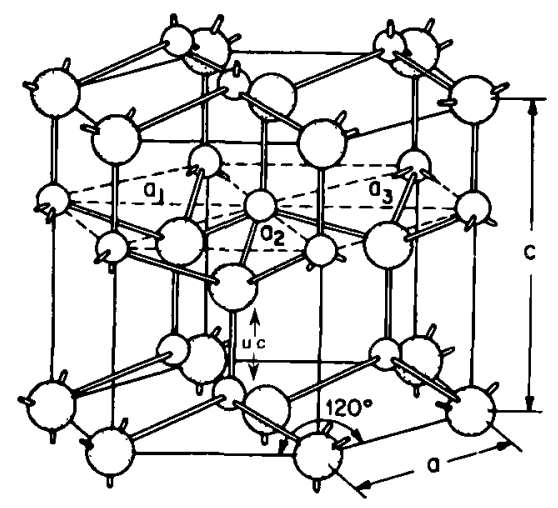

(a) Hexagonal Crystal System [7]
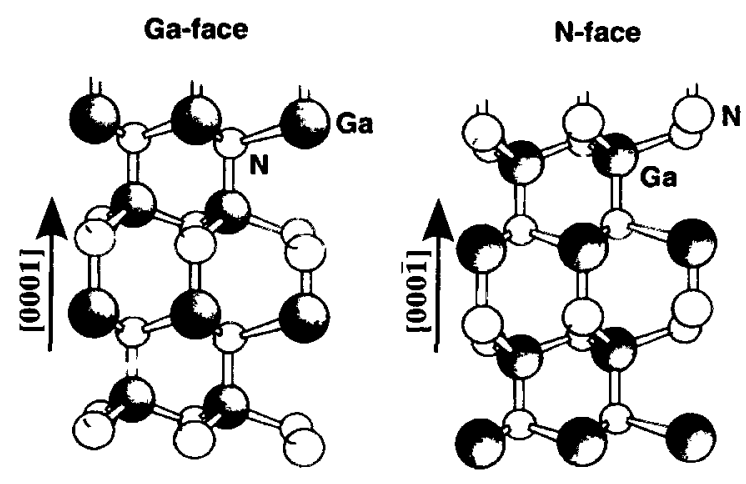

(b) GaN polarity [2]

Figure 2.3: Hexagonal crystal system and different polarities (Ga- and N-faced) of wurtzite GaN.

Growth of GaN single crystals of useful size remains a major challenge to this day. GaN is therefore usually grown epitaxially as a thin film on top of a range of suitable 
substrates. The most common GaN epitaxial growth techniques are Metal-Organic Vapour Phase Epitaxy (MOVPE, also known as MOCVD), Molecular Beam Epitaxy (MBE) and Hydride Vapour Phase Epitaxy (HVPE). The most common substrate materials for $\mathrm{GaN}$ are sapphire, $6 \mathrm{H}-\mathrm{SiC}$ and $\mathrm{Si}$. Some less common materials in use are diamond and AlN. $6 \mathrm{H}-\mathrm{SiC}$ has a lattice mismatch of only $3.5 \%$ with GaN (3.0806 $\AA$ versus $3.189 \AA$ for $\mathrm{GaN})$, similar thermal expansion coefficients $\left(4.46 \times 10^{-6} \mathrm{~K}^{-1}\right.$ versus $5.59 \times 10^{-6} \mathrm{~K}^{-1}$ for GaN) and a very high thermal conductivity coefficient. These properties make $6 \mathrm{H}-\mathrm{SiC}$ the best substrate for fabrication of high power $\mathrm{GaN}$ devices. The concentration of dislocations in even the best GaN epilayers is found to be large, $\sim 10^{10} \mathrm{~cm}^{-2}$. The negative impact of these defects on the operation of GaN transistors appears to be rather limited. As grown GaN is always n-type with carrier concentration $\sim 10^{16} \mathrm{~cm}^{-3}$. Unintentional doping with $\mathrm{Si}$, $\mathrm{O}$ or nitrogen vacancies is believed to be responsible for this phenomena $[2,8]$.

\subsubsection{Doping of GaN materials}

The numbers of free carriers in a semiconductor is dependent on the ionization energies of the donors/acceptors [9]:

$$
n \propto N_{d} T^{3 / 2} \exp \left(\frac{-E_{2}}{k T}\right)
$$

where $k$ is Boltzmann constant $\left(1.3806503 \times 10^{-23} \mathrm{~J} / \mathrm{K}\right), T$ is the absolute temperature in Kelvins, $E_{\imath}$ is the ionization energy, and $N_{d}$ is the donor/acceptor density.

The two most important elements used for intentional doping of GaN materials are $\mathrm{Si}$ and $\mathrm{Mg}$. $\mathrm{Si}$ is found to substitute on $\mathrm{Al}$ and Ga sites in $\mathrm{AlN}$ and GaN materials and acts as a shallow donor with ionization energies of $12-17 \mathrm{meV}$. The electrically active concentration of the donors/acceptors was found to be half the total dose. $\mathrm{Mg}$ acts as a shallow acceptor with ionization energy of 155-165 meV. Hydrogen plays 
an important role in passivating the $\mathrm{Mg}$ acceptors and Rapid Thermal Annealing (RTA) or electron irradiation is required to activate these dopants after the exposure to hydrogen. Both $\mathrm{Si}$ and $\mathrm{Mg}$ have moderate to low solubility in GaN and AlN materials [2].

Although donor and acceptor atoms can be incorporated during growth of GaN epilayers, selective area doping is not possible with this method. Additionally, heavy doping during growth increases epilayer defect densities. Ion implantation is an enabling technology for creating selective-area doping. The activation energy $E_{a}$ is the energy needed for implanted dopants to effectively substitute atomic sites in the crystal. The number of incorporated donors $N_{d}$ is related to anneal temperature by the following relationship [9]:

$$
N_{d} \propto N_{i m p} \exp \left(\frac{-E_{a}}{k T}\right)
$$

where $N_{i m p}$ is the implanted dopant concentration. Studies showed that good activation efficiency can be obtained at $1,100^{\circ} \mathrm{C}$, however ion induced damage cannot be completely removed until higher than $1,300^{\circ} \mathrm{C}$ temperatures are reached. Self compensation starts to occur at these high temperatures due to Si site switching with nitrogen atoms. Unprotected GaN and AlGaN surfaces undergo a significant degradation at these elevated temperatures. Si activation energies from 3.4 to $5.2 \mathrm{eV}$ were reported $[4,9]$.

An older, alternative method of semiconductor doping is solid or gas-phase doping, accomplished by establishing a high dopant concentration at the boundary of the semiconductor followed by a thermally driven diffusion. One major advantage of this method is the lack of crystal damage which occurs after ion implantation [10].

From a macroscopic point of view, diffusion can be described by the flow of atoms 
from areas of high concentration to areas with low concentration:

$$
F=-D \frac{\partial C}{\partial x}
$$

where $F$ is the flux of atoms in $\mathrm{cm}^{-2} \mathrm{~s}^{-1}, D$ is the diffusivity in $\mathrm{cm}^{2} \mathrm{~s}^{-1}$ and $\frac{\partial C}{\partial x}$ is the concentration gradient. From a microscopic point of view, the diffusivity $D$ depends exponentially on temperature $T$ and the diffusion activation energy $E_{d a}[10]$ :

$$
D=D_{\circ} \exp \left(\frac{-E_{d a}}{k T}\right)
$$

where $D_{o}$ is the diffusion coefficient. From this equation, smaller activation energies and larger diffusion temperatures result in faster diffusions. Several mechanisms can contribute to dopant diffusion at the same time, such as interstitial and substitutional diffusions, in which case Eq. 2.4 acquires additional terms. $E_{d a}$ in the case of substitutional diffusion and $E_{a}$ in the case of ion implanted dopant activation are not the same since the former one is the energy required for continuous substitutional process, and the latter one is the energy required for both an implanted atom (most likely in interstitial space) to acquire a site in the crystal lattice and for the repair of local damage. However, these two energies are related to the bond strength in the material.

Diffusion profiles for two cases resulting in the presence of an unlimited source of dopants (e.g. from a highly doped film) or fixed concentration at the surface (diffusion from a gas ambient with a concentration above the solid solubility of the dopant in the solid) are summarized below [10]:

$$
\begin{array}{lr}
C(x, t)=\frac{C_{0}}{2}\left[1-\operatorname{erf}\left(\frac{x}{2 \sqrt{D t}}\right)\right] & \text { Infinite source of dopants } \\
C(x, t)=C_{s}\left[1-\operatorname{erf}\left(\frac{x}{2 \sqrt{D t}}\right)\right] & \text { Constant surface concentration }
\end{array}
$$


where $\operatorname{erf}()$ is the error function defined by:

$$
\operatorname{erf}(z)=\frac{2}{\sqrt{\pi}} \int_{0}^{z} \exp \left(-x^{2}\right) \mathrm{d} x
$$

where $C \mathrm{o}$ is the concentration of an unlimited dopant source, $C_{s}$ is the constant surface concentration and $t$ is the diffusion time. $2 \sqrt{D t}$ is termed the diffusion length since it provides an extent of dopant profile motion. Subsequent anneals at different temperature with different anneal times result in $(D t)_{\text {eff }}$ which is given by [10]:

$$
(D t)_{\mathrm{eff}}=D_{1} t_{1}+D_{2} t_{2}+\ldots
$$

Dopants show very little movement in $\mathrm{GaN}$ materials even at temperatures of $1400^{\circ} \mathrm{C}$, with a diffusivity for $\mathrm{Si}$ found to be less than $10^{-13} \mathrm{~cm}^{2} \mathrm{~s}^{-1}$ at that temperature $[4,11]$. Due to high level of defects present in GaN epitaxial films, a diffusion, proceeding along grain boundaries and dislocations, with lower activation energies and higher diffusion constants is also possible $[12,13]$.

\subsubsection{High electron mobility transistors}

The high electron mobility transistors, also known as a Heterostructure Field Effect Transistor (HFET), is a field-effect transistor in which the carriers are confined to a thin sheet which is away from scattering centers. This carrier sheet is often abbreviated as a Two-Dimensional Electron Gas (2DEG) to emphasize the confinement of electrons. This electron confinement is usually achieved near the interface between the epitaxially grown layers of compound semiconductors which have different bandgap energy, so that the interface between them constitutes a heterojunction [14].

The initial concept of the accumulation of charge at a heterojunction interface and its potential for devices was introduced in late 1960s. The development in the 
1970s of high precision epitaxial-growth techniques made the fabrication of suitable high-quality heterostructure in the III-V compound semiconductors a reality. In 1978 a first GaAs heterostructure based device was demonstrated at Bell Laboratories [15]. The first AlGaN/GaN HEMT was demonstrated in 1993 shortly after the first GaN MESFET in 1992 by Asif Khan et al. [16,17].

An AlGaN/GaN HEMT structure can be seen in Fig. 2.4. In this structure $\mathrm{SiC}$

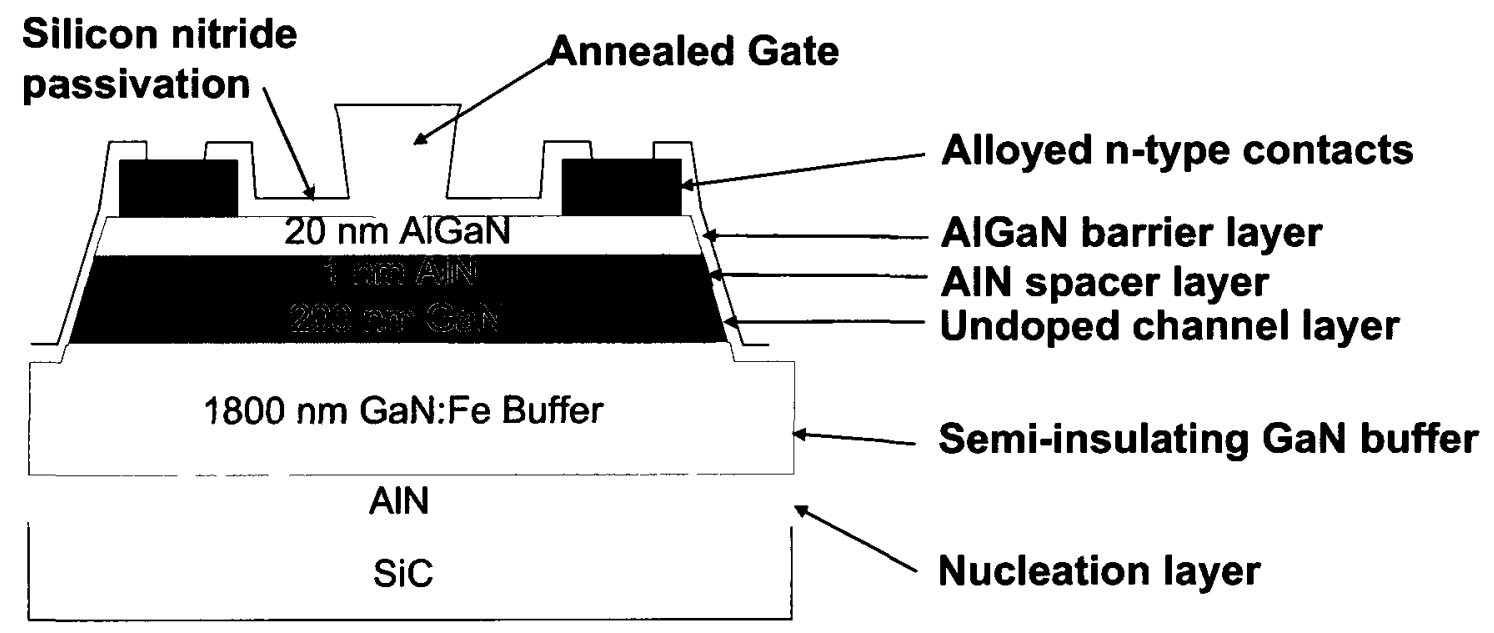

Figure 2.4: AlGaN/GaN HEMT Structure [18].

provides the substrate for GaN and AlGaN epilayers. A semi-insulating GaN buffer provides a foundation to grow GaN with relatively low defect densities while at the same time providing electrical isolation between adjacent devices on the chip. Semiinsulating properties are often accomplished by iron doping, which compensates unintentionally incorporated donor impurities such as $\mathrm{Si}, \mathrm{O}$ as well as native defects. The heterojunction of interest forms between an undoped channel layer of GaN and AlGaN often with an AlN spacer layer in-between. To form a useful heterojunction, the two materials must either have the same lattice spacing or be able to assume one common spacing. In AlGaN/GaN case the lattice spacing is not the same and thus strained epitaxial layers result, creating a pseudomorphic structure. The spacer 
between the two junctions, AIN, promotes a better quality interface which improves 2DEG confinement and reduces electron scattering in the channel. Alloyed ohmic contacts and a Schottky gate provide access and control over current in the 2DEG. Silicon nitride passivation addresses AlGaN surface states which negatively impact transistor performance.

To fully understand the role of stress on epilayers in GaN HEMTs and to explain the formation and location of the $2 \mathrm{DEG}$, some fundamental physical properties of III$\mathrm{N}$ materials need to be addressed. The Wurtzite structure is non-centrosymmetric and thus it is compatible with the existence of spontaneous polarization. As-grown III-N material possesses atomic layer-to-layer non-vanishing charge separation even in the absence of an external electric field, which can be denoted macroscopically as a polarization vector $\mathbf{P}$. The value of this spontaneous polarization in III-N materials is very large and increasing from GaN to InN and AlN, due to the increasing nonideality of the crystal structure ( $\mathbf{u}$ increases, $\mathbf{c} / \mathbf{a}$ decreases, and the difference from the ideal ratio of lattice constants of a closed packed hexagonal structure, $\mathbf{c} / \mathbf{a}=1.6333$, increases as well) [19]. The sign of the spontaneous polarization is found to be negative ( $\mathbf{P}$ points into the opposite to $\left[\begin{array}{llll}1 & 0 & 0 & 0\end{array}\right]$ direction) in III-Nitride semiconductors. In addition to spontaneous polarization, GaN possesses piezoelectric constants up to ten times larger than in GaAs based crystals. The source of piezoelectic polarization is the change to the lattice constants and to the associated change in $\mathbf{u}$ under the influence of induced strain. The orientation of the piezoelectic and spontaneous polarization is parallel in the case of tensile strain and antiparallel in the case of compressive strain in GaN or AlGaN layers. The spontaneous, piezoelectric and dielectric constants for AlN, GaN and InN can be found in Ambacher et al. [20].

Associated with the gradient of polarization in space is a polarization induced charge density given by $\rho_{p}=-\nabla P$. Fig. 2.5(a) depicts the crystal structure and polarization induced charges in Ga-faced and N-faced AlGaN/GaN HEMT structures. 


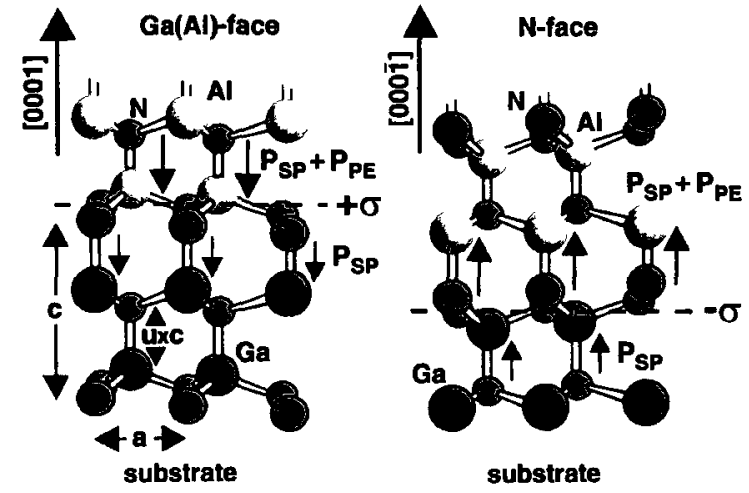

(a) Atomic level polarization in AlGaN/GaN

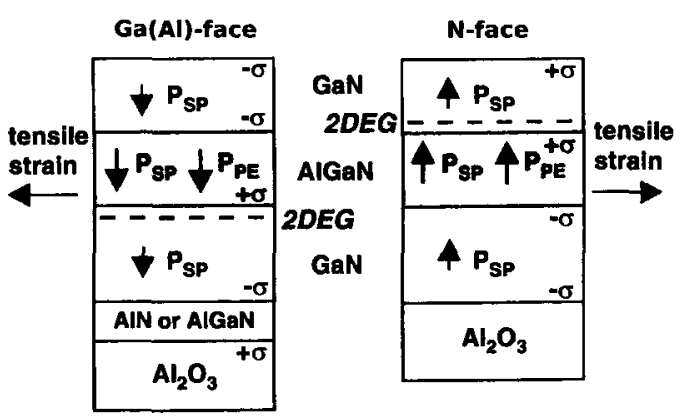

(b) Polarization in $\mathrm{AlGaN} / \mathrm{GaN}$ epilayers

Figure 2.5: Polarization and induced charges in crystal structure and epilayers in $\mathrm{GaN} / \mathrm{AlGaN} / \mathrm{GaN}$ Ga- and N-faced HEMTs [20].

At an abrupt interface of a heterostructure, the polarization can decrease or increase within a bilayer, causing a fixed polarization charge density. If the polarization induced charge density is positive $(+\sigma)$, free electrons will tend to compensate the polarization induced charge resulting in the formation of a 2DEG assuming that there is a source of electrons. In this case the Fermi level $E_{F}$ will drop below the triangular quantum well at the AlGaN/GaN interface (Fig. 2.6). For Ga-faced AlGaN/GaN heterostructure, polarization induced charge $\sigma$ can be expressed as:

$$
\sigma=P_{A l G a N}^{S P}+P_{A l G a N}^{P E}-P_{G a N}^{S P}
$$

Because the bandgap of GaN is smaller than of AlGaN, the 2DEG is always formed on the GaN side next to AlGaN/GaN heterostructure. However, depending on crystal polarity, 2DEGs are expected at the lower $\mathrm{AlGaN} / \mathrm{GaN}$ and upper GaN/AlGaN interfaces for Ga- and N-face GaN/AlGaN/GaN heterostructures respectively (Fig. 2.5(b)).

The bandgap, spontaneous and piezoelectric polarization constants as well as lattice constants and dopant activation energies depend on $\mathrm{Al}$ mole fraction in $\mathrm{AlGaN}$. 


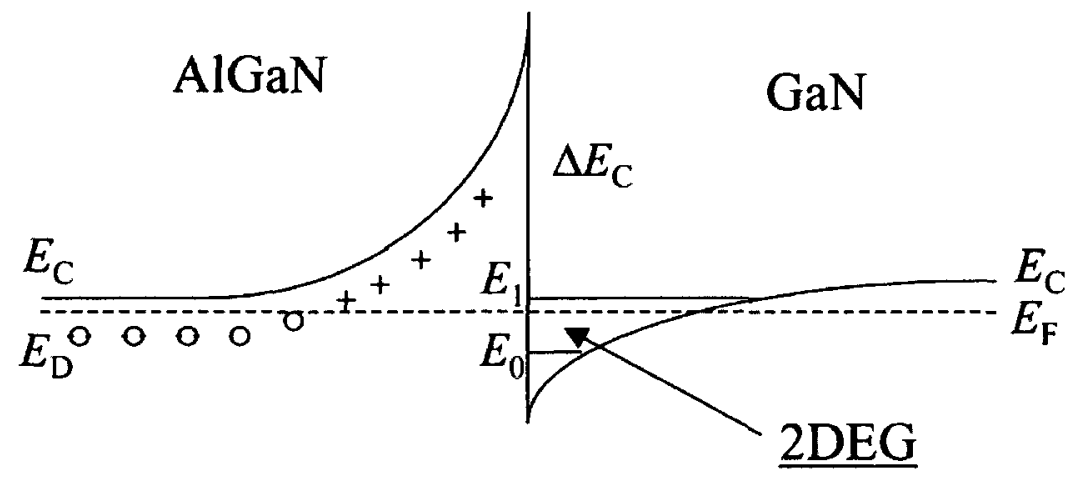

Figure 2.6: Conduction band diagram of AlGaN/GaN HEMT [4].

The $\mathrm{Al}$ mole fraction $x$ describes the ratio of $\mathrm{Al}$ to Ga in $\mathrm{AlGaN}$ material: $\mathrm{Al}_{x} \mathrm{Ga}_{1-x} \mathrm{~N}$. Some useful linear and quadratic interpolation models for AlGaN and AlGaN/GaN interface properties are summarized in the following equations ${ }^{1}[21,22]$ :

$$
\begin{aligned}
& a_{\text {AlGaN }}(x)=[3.189-0.086 x] \AA \\
& c_{A l G a N}(x)=[5.188-0.208 x] \AA \\
& u_{A l G a N}(x)=[0.3819 x+0.3772(1-x)-0.0032 x(1-x)] \\
& \varepsilon_{A l G a N}(x)=[0.03 x+10.28] \\
& E_{\text {AlGaN }}^{G}(x)=[6.13 x+3.42(1-x)-1.0 x(1-x)] \mathrm{eV} \\
& \Delta E^{C}(x)=\left[0.63\left(E^{G}(x)-E^{G}(0)\right)\right] \mathrm{eV} \\
& \chi_{A l G a N}(x)=\left[4.10-\frac{2}{3}\left(E^{G}(x)-E^{G}(0)\right)\right] \mathrm{eV} \\
& P_{A l G a N}^{S P}(x)=[-0.090 x-0.034(1-x)+0.021 x(1-x)] \mathrm{C} / \mathrm{m}^{2} \\
& P_{A l G a N^{2} / G a N}^{P E}(x)=[-0.0525 x+0.0282 x(1-x)] \mathrm{C} / \mathrm{m}^{2}
\end{aligned}
$$

where $a$ and $c$ are lattice constants, $u$ is the dimensionless lattice parameter, $\varepsilon$ is the relative permittivity, $E^{G}$ is the bandgap energy, $\Delta E^{C}$ is the conduction band offset, $\chi$ is the electron affinity, $P^{S P}$ and $P^{P E}$ are spontaneous and piezoelectric polarization

\footnotetext{
${ }^{1}$ note there are minor discrepancies for $\mathrm{GaN}$ polarization parameters in the literature

${ }^{2}$ pseudomorphically strained epilayer
} 
constants.

Spontaneous polarization makes a very large contribution to the total polarization in GaN materials. For instance, calculated $P^{S P}$ and $P^{P E}$ values from Eq. 2.17 and Eq. 2.18 for $d_{A l G a N}=30 \mathrm{~nm}$ and $x=0.3$ are: $P^{S P}=-1.28 \times 10^{-6} \mathrm{C} / \mathrm{cm}^{2}$ and $P^{P E}=-0.98 \times 10^{-6} \mathrm{C} / \mathrm{cm}^{2}$.

The sheet carrier concentration of the 2DEG located at the AlGaN/GaN interface of the nominally undoped structures is given by [23]:

$$
n_{s}(x)=\frac{\sigma(x)}{q}-\frac{\epsilon_{0} \epsilon(x)}{d q^{2}}\left[q \Phi_{B}(x)+E_{F}(x)-\Delta E^{C}(x)\right]
$$

where $\epsilon_{0}$ is the dielectric constant, $d$ is the AlGaN barrier width, $q$ is the electron charge, $q \Phi_{B}(x)$ is the surface barrier height, $E_{F}$ is the Fermi level position with respect to the GaN conduction-band edge, and $\Delta E^{C}$ is the conduction-band discontinuity between AlGaN and GaN. The 2DEG density thus can be directly controlled with $\mathrm{Al}$ mole fraction, since both $\sigma$ and $\Delta E^{C}$ increase with increase in $x$. The effect of $\mathrm{Al}$ concentration on bound interface density and sheet carrier concentration in a Gaface $\mathrm{AlGaN} / \mathrm{GaN}$ heterostructure with $d_{A l G a N}=30 \mathrm{~nm}$ can be seen in Fig. 2.7 [21], in which experimental results are compared with linear and non-linear polarization models. The highest measured and calculated sheet carrier concentration for a pseudomorphic AlGaN/GaN heterostructure is determined to be $2 \times 10^{13} \mathrm{~cm}^{-2}$ for $x=$ 0.37. For higher $\mathrm{Al}$ concentration, the $\mathrm{AlGaN}$ barrier starts to relax, diminishing the piezoelectric component.

Some issues still debated for AlGaN/GaN heterostructures include the determination of the Fermi-level position at the free surfaces and the origin of the electrons in the nominally undoped samples accounting for unusually high 2DEG densities. One of the possible models explaining the source of 2DEG is based on the existence of the surface donor-like states, proposed by Mishra [24]. Assuming the donor like states 


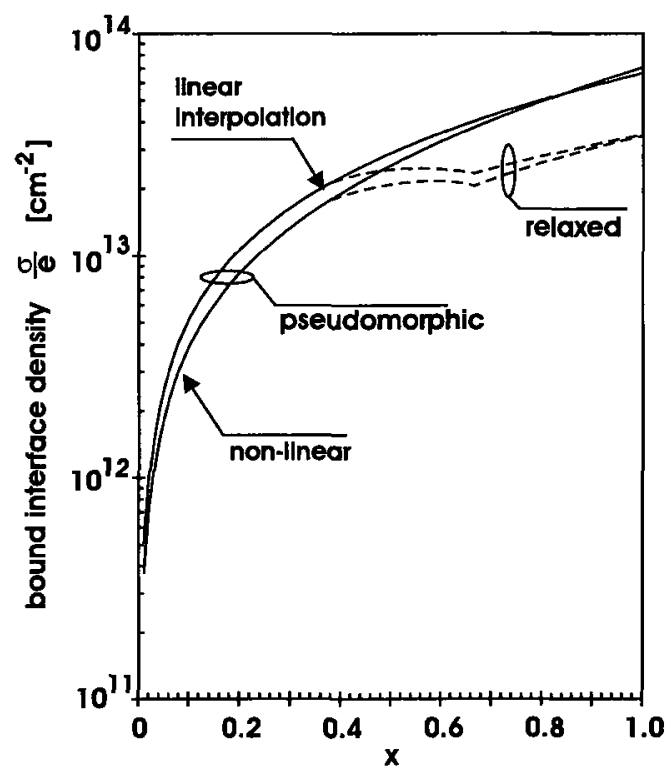

(a) Polarization induced bound charge

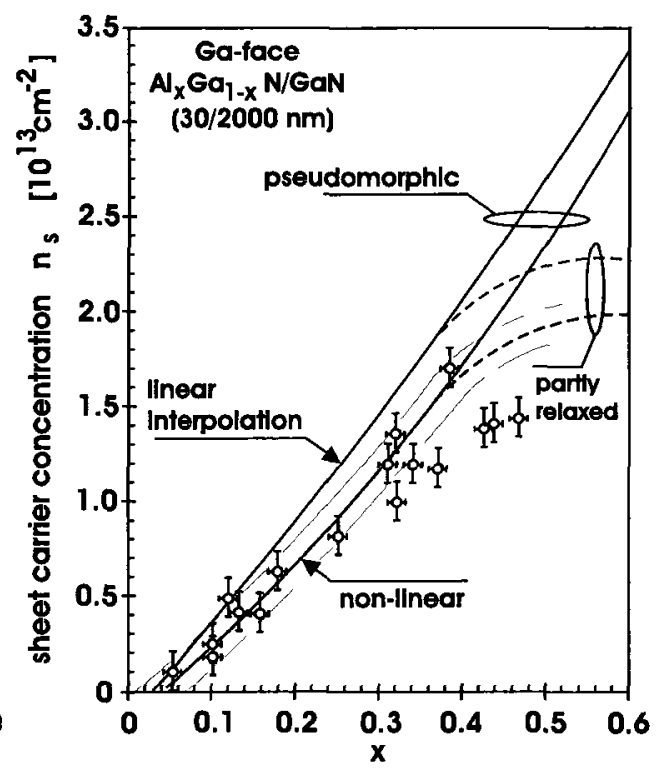

(b) 2DEG charge density

Figure 2.7: Polarization induced bound interface sheet densities and 2DEG density as a function of $\mathrm{Al}$ mole fraction for a Ga-face $\mathrm{AlGaN} / \mathrm{GaN}$ heterostructure $(30 / 2,000 \mathrm{~nm})$. Linear and non linear theoretical models and experimental results are compared [21].

are located deep in the AlGaN bandgap, they will all be occupied at a small value of $d$ (Fig. 2.8(a)). As the thickness of AlGaN increases, the Fermi level at the surface slides down, approaching and activating deep donors which in turn contribute to the formation of 2DEG. Therefore 2DEG density is expected to depend on the thickness of AlGaN barrier. This dependence is observed in both metallized and non-metallized structures. Fig. 2.8(b) provides additional theoretical and experimental sheet carrier concentrations dependence on $\mathrm{AlGaN}$ thickness.

For pseudomorphic HEMT structures with barrier thickness of more than $15 \mathrm{~nm}$, the nonlinear dependence of the 2DEG carrier concentration on the alloy composition (Eq. 2.19) can be approximated very well by [21]:

$$
n_{s}(x)=\left[-0.169+2.61 x+4.50 x^{2}\right] 10^{13} \mathrm{~cm}^{-2}, \quad x>0.06 .
$$




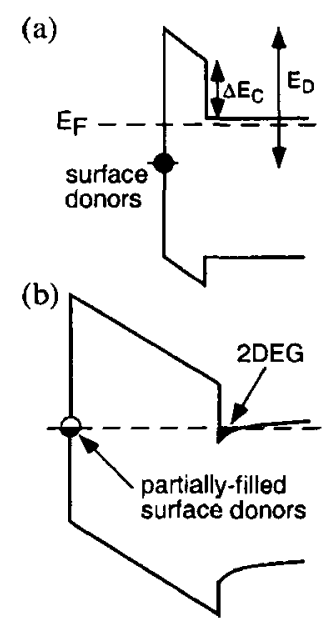

(c)

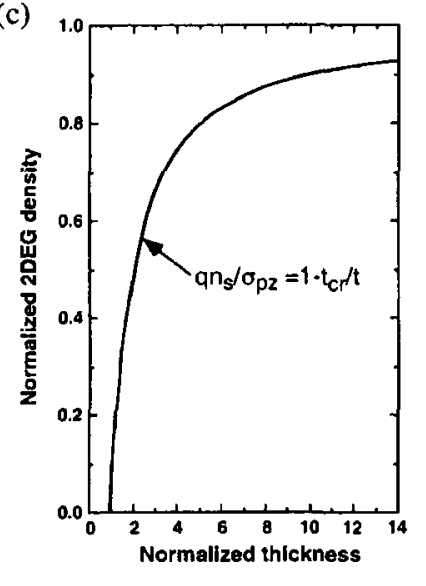

(a) Schematic band diagram illustrating the surface donor model with the undoped AlGaN barrier thickness less than (a) and greater than (b) the critical thickness for the formation of the 2DEG. Note the position of the Fermi level relative to the surface state in each case. (c) Calculated 2DEG density as a function of barrier thickness according to the surface donor mode [24].

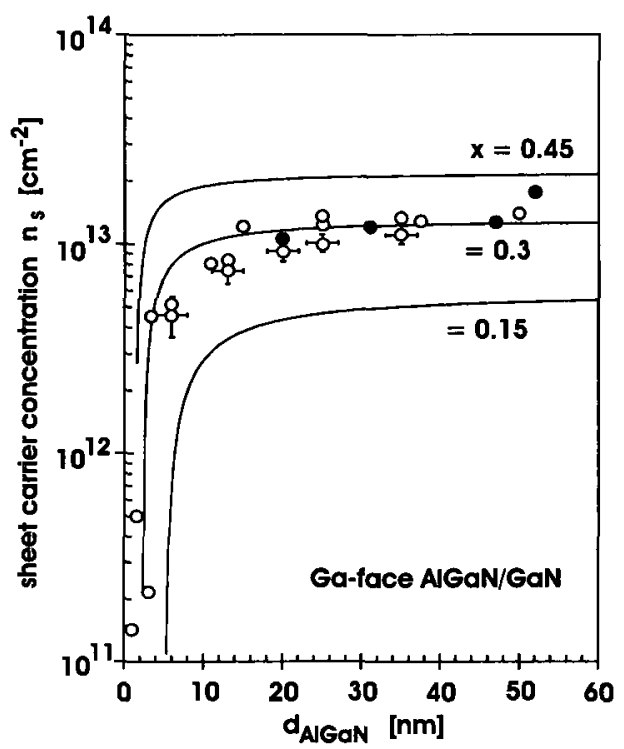

(b) Ambacher et al.: Theoretical dependence of the sheet carrier concentration on AlGaN thickness for $\mathrm{x}=0.15,0.30$ and $0.45 \mathrm{Al}$ mole fraction. Experimental results for $\mathrm{x}=0.30$ are shown with circles [21].

Figure 2.8: 2DEG density dependence on AlGaN layer thickness.

The electron mobilities are significantly higher in AlGaN/GaN heterojunctions than in the bulk GaN material since the 2DEG forms in an undoped GaN material which alleviates the ionized donor scattering mechanism. A thin AlN layer enhances this effect even further by reducing the electron penetration into the AlGaN due to the higher effective conduction band discontinuity (Fig. 2.9) and also the binary AlN at the interface has no alloy disorder scattering. In addition, the larger conduction band discontinuity results in a small increase in 2DEG concentration [25]. Room temperature mobilities of $2,000 \mathrm{~cm}^{2} /(\mathrm{Vs})$ are commonly achieved. Very high saturation velocities of $3 \times 10^{7} \mathrm{~cm} / \mathrm{s}$ are predicted with Monte Carlo simulations (Fig. 2.10). 


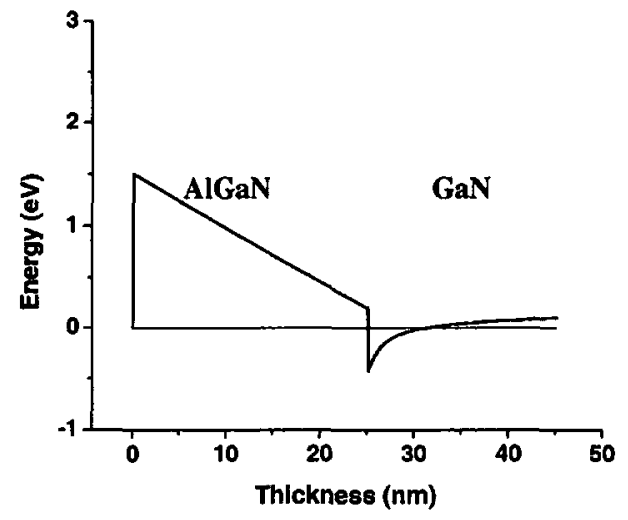

(a) $\mathrm{AlGaN} / \mathrm{GaN}$ heterostructure

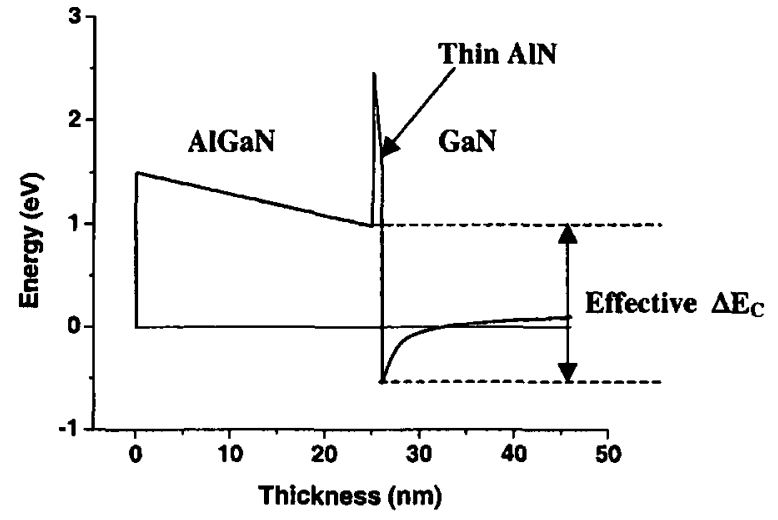

(b) AlGaN/AlN/GaN heterostructure

Figure 2.9: Conduction band diagram of $\mathrm{AlGaN} / \mathrm{GaN}$ and $\mathrm{AlGaN} / \mathrm{AlN} / \mathrm{GaN}$ heterostructures. Dipole in AIN increases the effective $\Delta E^{C}\left(\Delta E_{C}\right.$ in the figure) $[25]$.

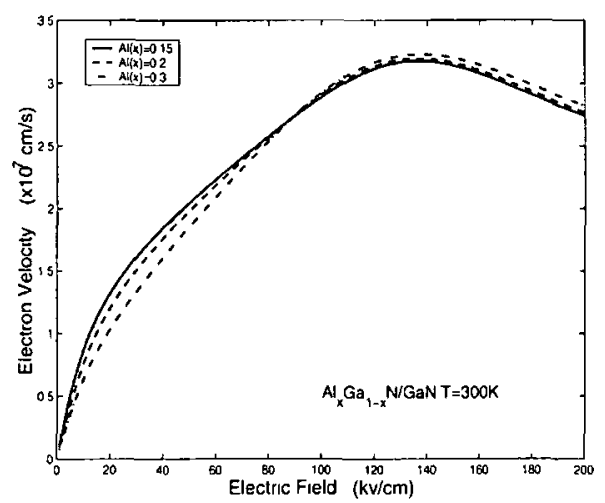

Figure 2.10: Calculated electron velocity vs electric field for AlGaNGaN HEMT structures with $\mathrm{Al}$ mole fractions of $0.15,0.2$ and 0.3 [26].

Very high 2DEG sheet carrier concentration together with the high electron mobilities materialize into a low 2DEG sheet resistance $R_{s}$, advantageous for the performance of HEMT devices:

$$
R_{s}=\frac{1}{\mu n_{s} q}
$$

Mishra's model provides an insight into the importance of surface states for the 2DEG densities. Regardless of the origin of the source of 2DEG, negative charge 
trapped on the surface of AlGaN will negatively affect the 2DEG at the heterostructure by electrostatically repelling it away. Thus, even when the thickness of AlGaN is sufficiently large to support high 2DEG concentrations, the surface conditions can play an enormously important role in the final density of electrons found at the heterojunction.

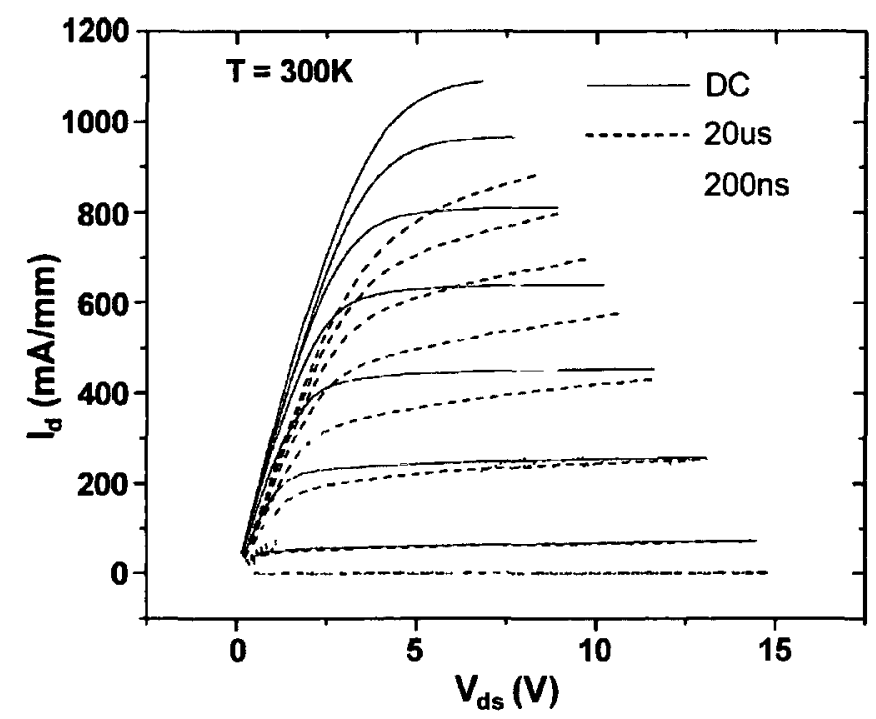

Figure 2.11: DC and pulsed I-V characteristics of an unpassivated AlGaN/GaN HEMT on SiC substrate. Current collapse (DC to RF dispersion) could be observed in the pulsed mode [1].

Simple analytic charge control models are quite poor for AlGaN/GaN HEMTs, mainly because of many effects present in these devices. The best models rely on numerical solutions to Schroedinger-Poisson equations. AlGaN/GaN HEMTs operation and control equations are similar to that of AlGaAs/GaAs. The dependence of the sheet charge $n_{s}$ on the applied gate voltage $V_{g}$ as well as the threshold voltage $V_{t h}$ for 
AlGaAs/GaAs heterostructures are given by [15]:

$$
\begin{aligned}
n_{s} & =\frac{\epsilon}{q\left(d_{d}+d_{\imath}+\Delta d\right)}\left(V_{g}-V_{t h}\right) \\
& =\frac{\epsilon}{q\left(d_{\text {barrier }}+\Delta d\right)}\left(V_{g}-V_{t h}\right)
\end{aligned}
$$

where

$$
V_{t h}=\phi_{b}-\Delta E^{C}-\frac{q N_{d} d_{d}^{2}}{2 \epsilon}+\Delta E_{F O}
$$

$d_{d}$ is the AlGaAs donor-layer thickness, $d_{\imath}$ is the AlGaAs spacer-layer thickness, $\phi_{b}$ is the Schottky barrier height between gate metal and AlGaAs, $N_{d}$ is the dopant concentration in the donor layer, and $\Delta d$ and $\Delta E_{F O}$ are fitting parameters. Typical AlGaN/GaN HEMTs operate in depletion mode: their threshold (pinch off) voltage is negative. The drain current $I_{d}$ is directly proportional to the sheet charge density, gate width and drift velocity [27]. Although there are significant physical differences between HEMTs and metal-oxide-semiconductor field-effect transistor, the DC I-V curves are similar for both devices (Fig. 2.11). Pulsed I-V curves are indicative of transistor's RF performance and are prone to a so called "current collapse" phenomena.

\subsubsection{Current collapse and silicon nitride passivation}

Surface passivation is a fundamental concern in AlGaN/GaN HEMTs technology which alleviates the current-collapse (DC to RF dispersion) phenomena. Current collapse originates from trapping effects at the device surface and/or in the buffer, strongly limiting the device performance under high-frequency operation by reducing the $\mathrm{RF}$ transconductance with respect to the DC transconductance [28]. This phenomena can be subdivided into the gate lag and the drain lag related current 
collapse.

Fig. 2.11 depicts I-V curves demonstrating the effect of gate lag related current collapse in unpassivated $\mathrm{AlGaN} / \mathrm{GaN}$ transistor. Current collapse is mostly eliminated using a thick $(\sim 100 \mathrm{~nm})$ silicon nitride $\left(\mathrm{Si}_{3} \mathrm{~N}_{4}\right.$ or $\left.\mathrm{SiN}\right)$ deposited film, which significantly reduces the amount of surface traps available on AlGaN surface. One of the mechanism thought to be responsible for the reduction of surface traps relies on Si acting as a surface donor and passivating the traps with the donated electrons.

\subsubsection{Metal contacts to semiconductor materials}

A metal-semiconductor junction can produce two types of contacts: ohmic contacts and rectifying (Schottky) contacts. An ohmic contact displays a linear current/voltage characteristic (Fig. 2.12(a)). A Schottky contact displays a rectifying behaviour, conducting current for only one polarity of voltage bias (Fig. 2.12(b)). Low resistance ohmic contacts are essential for the efficient operation of high power electronic devices, in particular to achieve high current densities, high extrinsic gain and low Joule heating loss to allow high temperature operation [29]. High frequency operation is also degraded by poor ohmic contacts as RC time constant increases.

A Schottky contact with a low reverse bias leakage current and high turn on voltage is essential between the gate metal and the semiconductor for superior HEMT operation. High power diodes have different requirements for the Schottky contact: low reverse bias current, high forward bias current and low turn on voltage.

Depending on the barrier height and width, three electron transport mechanisms can occur at a free metal boundary. If the thermal energy of the electrons at the Fermi level is sufficient to overcome the barrier, Thermionic Emission (TE) occurs. A high barrier sufficiently lowered in the presence of an electric field can gives rise to a Thermionic Field Emission (TFE). In the presence of a strong electric field $E$ the potential barrier outside the surface may bend sufficiently steeply and can become 


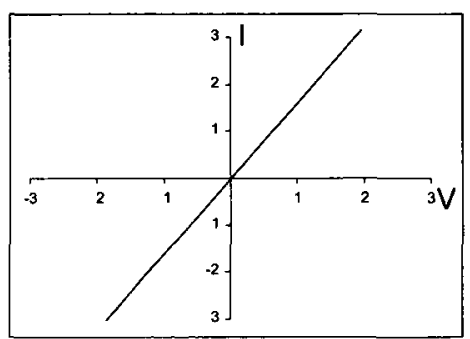

(a) Ohmic behaviour

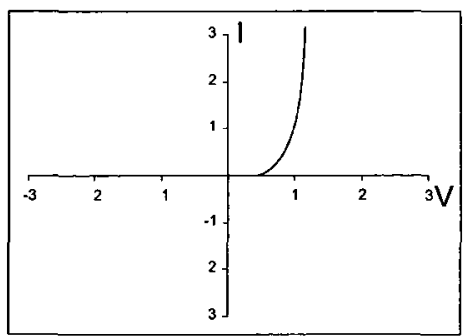

(b) Schottky behaviour

Figure 2.12: I-V characteristic curves for ohmic and Schottky contacts.

very narrow to allow high probabilities for the electrons in the metal to tunnel through. This tunnelling is temperature independent and is termed Field Emission (FE). The current densities of escaping electrons for each case are summarized below [30]:

$$
\begin{array}{lr}
J \approx A^{* *} T^{2} \exp \left(-\frac{q \Phi_{m}}{k T}\right) & \text { for Thermionic Emission } \\
J \approx A^{* *} T^{2} \exp \left[-\frac{\left(q \Phi_{m}-\beta_{S} E^{\frac{1}{2}}\right)}{k T}\right] & \text { for Thermionic Field Emission } \\
J \approx C E^{2} \exp \left(-\frac{E_{c}}{E}\right) & \text { for Field Emission }
\end{array}
$$

where $A^{* *}=2 \pi q m^{*} k^{2} / h^{3}$ is the effective Richardson constant, $\beta_{S}=\left[e^{3} / 4 \pi \varepsilon_{o}\right]^{\frac{1}{2}}$ is the Schottky coefficient, $h$ is the Planck's constant, $m^{*}$ is the effective electron's mass, $q$ is the electron charge, $k$ is the Boltzmann constant, $T$ is the absolute temperature and $\Phi_{m}$ is the work function of the metal. $C$ and $E_{c}$ are temperature independent constants that depend on metal work function and electron's effective mass.

When the metal is brought in contact with the surface of an n-type semiconductor the Fermi levels in both materials align. The difference between the metal work function $q \Phi_{m}$ and semiconductor electron affinity $q \chi_{s}$ is defined as the Schottky Barrier Height $(\mathrm{SBH}), q \Phi_{B}[31]$ :

$$
\Phi_{B}=\Phi_{m}-\chi_{s}
$$

$\mathrm{SBH}$ can be seen as the energy an electron in the metal must have to cross the barrier 
into the semiconductor conduction band. On the other hand the built in potential $V_{b i}$ is the barrier for electrons on semiconductor side (Fig. 2.13) [31].

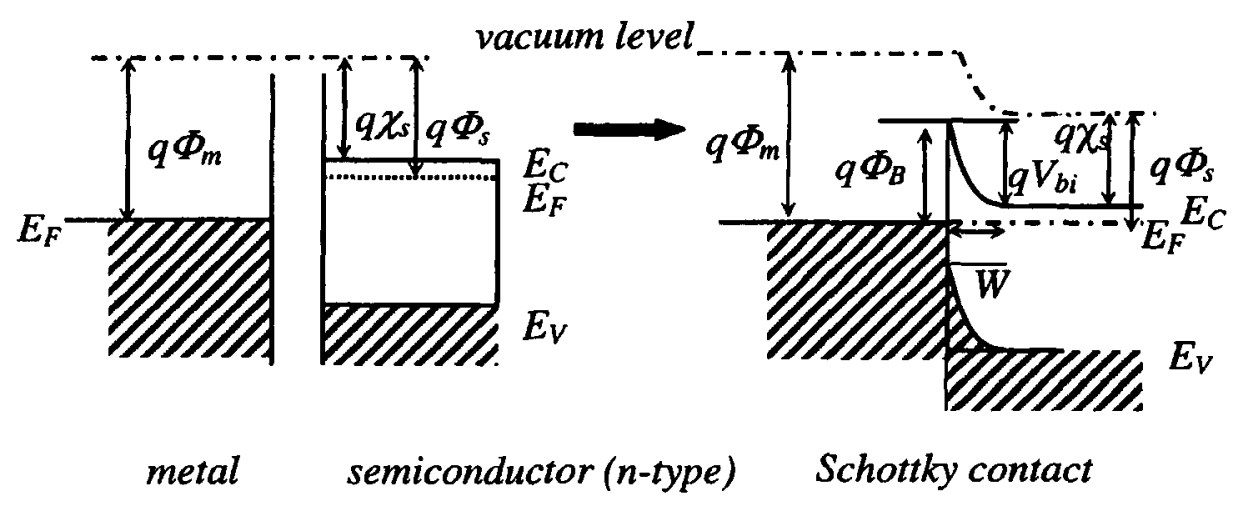

Figure 2.13: Schottky contact formation [31].

If the work function of the metal is higher than that of an n-type semiconductor, a rectifying contact will form. For lightly doped semiconductors $\left(N<1 \times 10^{17} \mathrm{~cm}^{-3}\right)$ the main conduction mechanism is thermionic emission, where the carriers have sufficient thermal energy to surmount the Schottky barrier and pass from a material to the other. In this case, the application of thermionic emission theory at the semiconductor junction interface in the presence of voltage $\mathrm{V}$ yields the following current density $\mathrm{J}$ :

$$
J=A^{* *} T^{2} e^{\frac{-q \Phi_{B}}{k T}}\left(e^{\frac{q V}{n k T}}-1\right)
$$

where $\mathrm{n}$ is the empirical ideality factor. The ideality factor provides a measure of the quality of a metal-semiconductor junction and is highly process dependent. An ideal Schottky diode will have an ideality factor of 1 . Any electron transport mechanism other than thermionic will make the ideality factor larger. It is generally accepted that when $n \ll 1.2$, the thermionic model applies and the plot of $\ln (\mathrm{J})$ vs $\mathrm{V}$ can be used to accurately extract the saturation current density and the ideality factor [32]. Image force barrier lowering, electron tunnelling and generation-recombination in depletion region are some of the reasons why Schottky diodes often do not exhibit 
a well defined saturation current density under reverse bias conditions [33]. Usually the total current can be expressed as a sum of several current components at the junction [34]:

$$
I_{T O T}=I_{T E}+I_{F E}+I_{G R}+I_{L K}
$$

where $I_{T E}$ is the current due to thermionic emission, $I_{F E}$ is field emission current, $I_{G R}$ is generation-recombination current and $I_{L K}$ is the leakage current.

When the metal work function is lower than the work function of the semiconductor, the contact can be made ohmic by band aligning (Fig. 2.14). This is, however, rarely possible due to the lack of suitably low work function metals (Table 2.2).

\begin{tabular}{ccc}
\hline \hline Material & Work Function $(\mathrm{eV})$ & Resistivity $(\Omega \mathrm{cm})$ \\
\hline $\mathrm{Ga}$ & 3.96 & - \\
$\mathrm{Al}$ & 4.28 & $2.65 \times 10^{-6}$ \\
$\mathrm{Ti}$ & 4.33 & $4.20 \times 10^{-6}$ \\
$\mathrm{Ni}$ & 5.15 & $6.84 \times 10^{-6}$ \\
$\mathrm{Au}$ & 5.10 & $2.35 \times 10^{-6}$ \\
$\mathrm{Ta}$ & 4.25 & - \\
$\mathrm{Pd}$ & 5.12 & $1.08 \times 10^{-5}$ \\
$\mathrm{Pt}$ & 5.65 & $1.01 \times 10^{-5}$ \\
$\mathrm{Ir}$ & 5.62 & $1.00 \times 10^{-5}$ \\
$\mathrm{Re}$ & 4.72 & $2.25 \times 10^{-4}$ \\
$\mathrm{TiN}$ & 3.74 & - \\
$\mathrm{AlGaN}$ & $<4.10$ & - \\
\hline \hline
\end{tabular}

Table 2.2: Work functions and resistivity of several metals suitable for Schottky junctions $[2,35]$.

For many III-V materials SBH is almost independent of metal work function due to the formation of interfacial metal-semiconductor dipoles, the presence of surface states with energies in the forbidden gap and the formation of new metal-semiconductor 


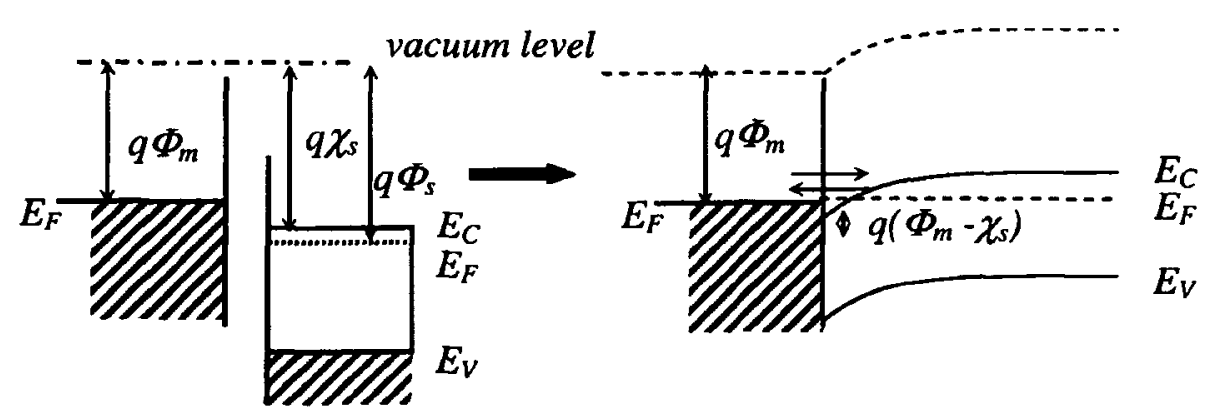

metal semiconductor (n-type) Ohmic contact by band alignment

Figure 2.14: Ohmic contact formation [31].

alloys at the interface [33]. To account for these effects, SBH can be modelled using the following equation:

$$
\Phi_{B n}=\gamma\left(\phi_{m}-\chi_{s}\right)+(1-\gamma)\left(E^{G}-\phi_{o}\right)
$$

where

$$
\gamma=\frac{\epsilon_{\imath}}{\epsilon_{\imath}+q \delta D_{s}}
$$

$\delta$ and $\epsilon_{\imath}$ is the thickness of the interface layer and its permittivity, $D_{s}$ is the density of surface states assumed to be uniformly distributed in the energy within the bandgap up to the level $\phi_{o}$ measured from the top of the valance band. The dependence of the $\mathrm{SBH}$ on the bandgap value is particularly problematic in wide bandgap semiconductors in which the barrier tends to be high regardless of the value of $\phi_{m}-\chi_{s}$, so making a good ohmic contact is difficult. At the same time, because of the inapplicability of the simple Schottky Barrier model (Eq. 2.27), it is also very difficult to achieve a high SBH to make a good Schottky diode.

By far the most common way of achieving an ohmic contact between metal and semiconductor is by creating a heavily doped region at the metal-semiconductor interface. High doping reduces the barrier height via barrier image force lowering 
$q \Delta \Phi_{B}$, but the effect is fairly weak, $\Delta \Phi_{B} \propto N_{d}^{\frac{1}{4}} V_{b n}^{\frac{1}{4}}$. The doping, however, also reduces the width of the barrier $W$, where $W \propto N_{d}^{-\frac{1}{2}}$. For high doping concentrations $\left(N_{d}>1 \times 10^{19} \mathrm{~cm}^{-3}\right)$ the electrons at the Fermi level can have enough energy to tunnel through the barrier and a tunnelling ohmic contact forms (Fig. 2.15). The carrier transport is ruled by the field emission mechanism.

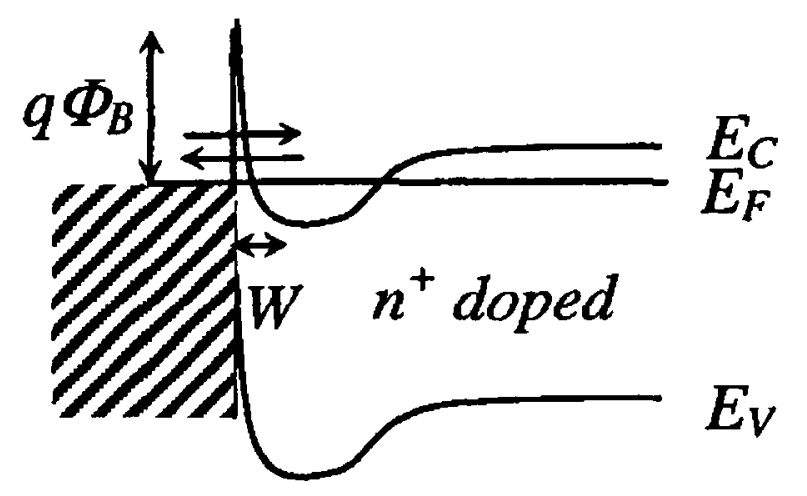

Figure 2.15: Tunnelling ohmic contact band diagram [31].

An intermediate mechanism of the current transport through the barrier occurs for doping levels in the range of $10^{17}$ to $10^{19} \mathrm{~cm}^{-3}$, for which electrons at higher than Fermi energy levels can tunnel through the barrier, and is termed the thermionic field emission. The parameter which determines the dominant conduction mechanism is [31]:

$$
E_{00}=\frac{h}{4 \pi}\left(\frac{N_{d}}{m^{*} \varepsilon_{s}}\right)^{\frac{1}{2}}
$$

where $h$ is the Planck's constant, $m^{*}$ is the effective electron mass and $\varepsilon_{s}$ is the dielectric constant of the semiconductor. Electron transport mechanisms are summarized below: 


$$
\begin{array}{ll}
N_{d} \ll 1 \times 10^{17} \mathrm{~cm}^{-3} & \rightarrow k T \gg q E_{00} \quad(\mathrm{TE}) \\
N_{d} \gg 1 \times 10^{19} \mathrm{~cm}^{-3} & \rightarrow k T \ll q E_{00} \quad(\mathrm{FE}) \\
N_{d} \approx 1 \times 10^{17}-1 \times 10^{19} \mathrm{~cm}^{-3} \rightarrow k T \approx q E_{00} \quad(\mathrm{TFE})
\end{array}
$$

A general specific contact resistivity at the junction as a function of current density is given by [31]:

$$
\rho_{c}=\left(\frac{\partial J}{\partial V}\right)_{V=0}^{-1}
$$

When this expression is applied to the thermionic current density across the metalsemiconductor junction given in Eq. 2.28, the following specific contact resistivity is obtained:

$$
\rho_{c \jmath}=\frac{k}{q A^{* *} T} \exp \left(\frac{q \Phi_{B}}{k T}\right)
$$

When thermionic field emission and field emission are considered, the following relations result:

$$
\begin{array}{lr}
\rho_{c \jmath} \propto \exp \frac{\Phi_{B}}{E_{00} \operatorname{coth}\left(\frac{E_{00}}{k T}\right)} & \text { for thermionic field emission } \\
\rho_{c \jmath} \propto \exp \left(\frac{\Phi_{B}}{E_{00}}\right) \propto \exp \left(\frac{\Phi_{B}}{N_{d}}\right) & \text { for field emission }
\end{array}
$$

As can be seen from these equations, the specific contact resistivity in tunnelling contacts is strongly dependent on both barrier height and doping concentration.

The total contact resistance between the ohmic metal and the channel in the device can be written as:

$$
R_{c . t o t}=R_{c \jmath}+R_{m e t}+R_{c s e m}
$$


where

$$
R_{c \jmath}=\frac{\rho_{c \jmath}}{A_{\mathrm{eff}}}
$$

is the resistance associated with the metal-semiconductor junction, $A_{\text {eff }}$ is the effective contact area, $R_{\text {met }}$ is the resistance associated with the ohmic metal and $R_{c s e m}$ is the resistance associated with the semiconductor material. An ohmic contact will have a negligible $R_{\mathrm{c} J}$ resistance in comparison with the other two.

Theoretical calculations do not predict contact resistances well, thus it is often necessary to extract the parameters from measurements on fabricated structures. This is usually done using Transmission Line Model (TLM) structures (Fig. 2.16). The resistance measured between rectangular ohmic patches follows the equation:

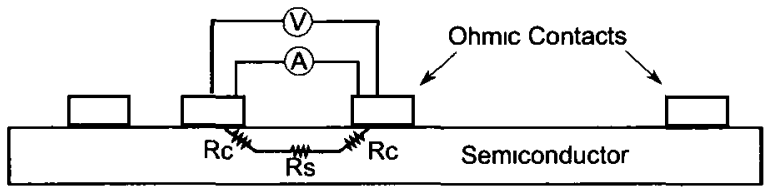

(a) Side view

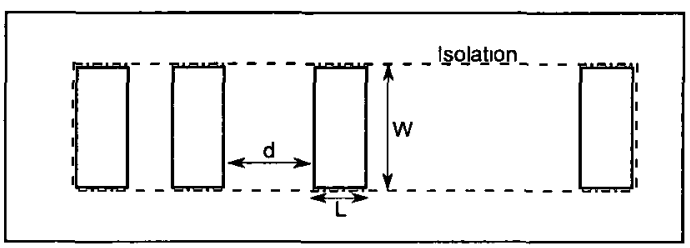

(b) Top view

Figure 2.16: TLM test structure.

$$
R(d)=2 \frac{R_{c}}{W}+\frac{R_{s}}{W} d
$$

where $R_{c}$ is the width independent ohmic contact resistance, often conveniently expressed in units of $\Omega-\mathrm{mm}, W$ is the width of the rectangular contact, $R_{s}$ is the sheet resistance between the contacts and $d$ is the contact spacing. The fit of a straight line to the measured data is used to extract both $R_{c}$ and $R_{s}$ parameters (Fig. 2.17).

For lateral ohmic contacts an effective conducting contact length, or transfer length, is defined as:

$$
L_{t}=\left(\frac{\rho_{c}}{R_{s c}}\right)^{\frac{1}{2}}
$$




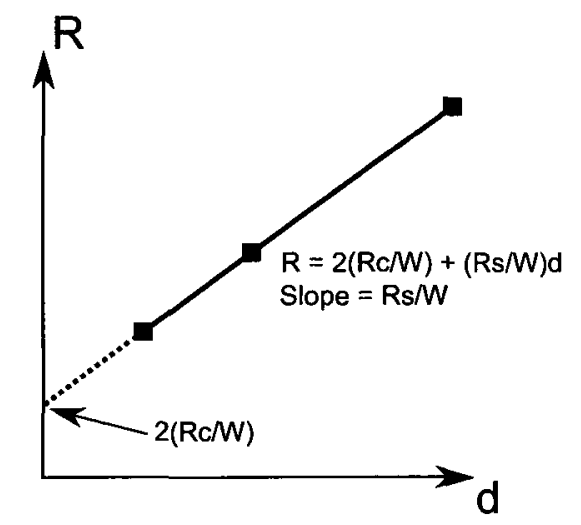

Figure 2.17: Resistance measured between adjacent TLM structure contacts versus their spacing.

where $\rho_{c}$ is the specific ohmic contact resistivity and $R_{s c}$ is the sheet resistance of the semiconductor below the contact. Note that $R_{s c}$ does not have to be equal to $R_{s}$. Transfer length can be interpreted as the distance at which the current density at the lateral contact drops by a factor of $e^{1}$. The contact resistance can be found by the following relation [36]:

$$
R_{c}=\frac{\sqrt{\rho_{c} R_{s c}}}{\tanh \left(\frac{L}{L_{t}}\right)}
$$

For $L \gg L_{t}$, the effective area of the contact is defined by $L_{t}$, thus Eq. 2.40 can be rearranged to get:

$$
R_{c}=\frac{\rho_{c}}{L_{t}}
$$

For $L \ll L_{t}$, the effective area of the contact is defined by $L$, thus Eq. 2.40 becomes:

$$
R_{c}=\frac{\rho_{c}}{L}
$$

A modified TLM method, referred to as circular-TLM (c-TLM), uses circular ohmic pads surrounded by rings of ohmic metal (Fig. 2.18). The advantage of this method lies in avoiding the need for mesa isolation to define the boundaries of the conduction layer. 


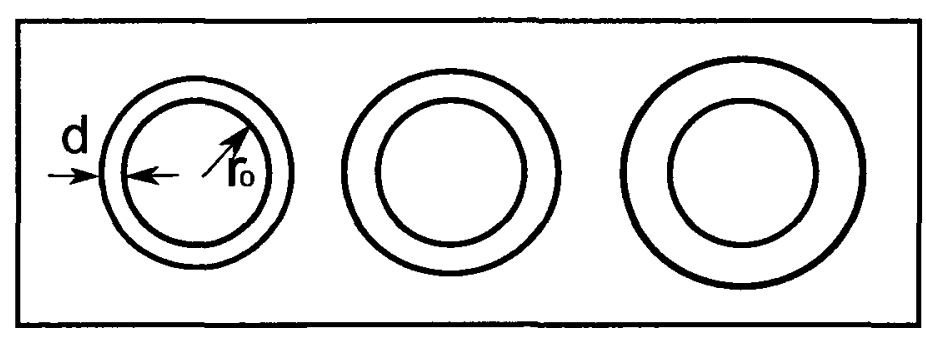

Figure 2.18: c-TLM structure.

For a transfer length significantly smaller than the dimension of the contact pad $r_{0}, \mathrm{c}-\mathrm{TLM}$ resistance can be modelled using the following equation [37]:

$$
\begin{aligned}
R(d) & \approx \frac{R_{s}}{2 \pi}\left[\ln \left(\frac{r_{0}+d}{r_{0}}\right)\right]+\frac{R_{s c}}{2 \pi}\left[L_{t}\left(\frac{1}{r_{0}+d}+\frac{1}{r_{0}}\right)\right] \\
& =\frac{R_{s}}{2 \pi}\left[\ln \left(\frac{r_{0}+d}{r_{0}}\right)\right]+\frac{R_{c}}{2 \pi}\left[\frac{1}{r_{0}+d}+\frac{1}{r_{0}}\right]
\end{aligned}
$$

By fitting this equation to c-TLM measured data, contact resistance $R_{c}$ and the sheet resistance $R_{s}$ can be extracted.

\subsubsection{Ohmic and Schottky contacts to GaN and AlGaN/GaN}

A Ti/Al based metal stack has traditionally been used to achieve good ohmic contacts on $\mathrm{GaN}$ and AlGaN layers. A specific contact resistivity in the range of $10^{-7}$ to $10^{-6}$ $\Omega$ - $\mathrm{cm}^{2}$ was obtained by several groups [29]. Both $\mathrm{Ti}$ and $\mathrm{Al}$ have a fairly low work function, Ti has a very good adhesion properties to $\mathrm{GaN}$ and upon alloying reduces native oxides formed on the GaN surface. Solid phase reaction between Ti and GaN forming $\mathrm{TiN}$ is possible since the heat of formation of $\mathrm{TiN}$ and $\mathrm{GaN}$ are -265 and $-110.9 \mathrm{~kJ} / \mathrm{mol}$ respectively. Out diffusion of nitrogen creates donor vacancies in GaN and AlGaN [38]. Only 2 monolayers of TiN formed at the interface are sufficient to generate a $10 \mathrm{~nm}$ thick layer of GaN with a doping concentration of $10^{20} \mathrm{~cm}^{-3}$ which is suitable for tunnelling contacts [39]. In addition, TiN has lower than $4 \mathrm{eV}$ work function, which would assist tunnelling. The addition of $\mathrm{Al}$ allows for lower ohmic 
contact resistances than those obtained with Ti layer alone. The exact mechanism by which $\mathrm{Al}$ reduces the contact resistance is unclear. There are indications that $\mathrm{Al}$ reduces the reactivity of $\mathrm{Ti}$ towards GaN or AlGaN layers and this prevents undesired contact features, such as voids, from forming [40]. Luther et al. [41] proposed that $\mathrm{Al}$ diffuses through the $\mathrm{Ti}$ layer, resulting in an ohmic contact when a low work function Al-Ti inter-metallic phase comes into contact with the GaN surface.

Both $\mathrm{Ti}$ and $\mathrm{Al}$ have a high propensity towards oxidation even at low oxygen concentrations. Additionally, $\mathrm{Al}$ tends to ball up during alloying ( $\mathrm{Al}$ melting point is around $660^{\circ} \mathrm{C}[29]$ ), resulting in a rough surface morphology of the Ti/ $\mathrm{Al}$ contacts. In order to minimize this problem, a Ti/Au overlayer (melting temperatures of $1,600^{\circ} \mathrm{C}$ and $1,060^{\circ} \mathrm{C}$ respectively) is commonly used on top of standard $\mathrm{Ti} / \mathrm{Al}$ metallization. An additional $\mathrm{Ti}$ layer helps to tie up excess $\mathrm{Al}$, preventing it from balling up [39]. In general, annealing of the four layer ohmic metal stack results in the formation of many alloys ( $\mathrm{TiN}, \mathrm{TiAl}, \mathrm{AlN}$ and $\mathrm{Al}_{2} \mathrm{Au}$ ) and the final contact resistance is very sensitive to the individual layer thickness as well as anneal conditions [29]. Typical anneal temperatures for $\mathrm{Ti} / \mathrm{Al} / \mathrm{Ti} / \mathrm{Au}$ ohmic metal schemes range from 800 to $900^{\circ} \mathrm{C}$. As of $2002 \mathrm{Ti} / \mathrm{Al} / \mathrm{Ti} / \mathrm{Au}$ contact resistance ranged from 0.7 to $2.3 \mathrm{Ohm}-\mathrm{mm}$ [42]. More recently ohmic contact resistances to GaN and AlGaN material range from 0.5 to 1 Ohm-mm with some experimental stacks reaching $0.3 \mathrm{Ohm}-\mathrm{mm}$ and lower values [29].

It should be noted that a trade-off exists between the ohmic contact resistance and the 2DEG sheet resistance values in AlGaN/GaN heterostructures. Thick AlGaN epilayers with high $\mathrm{Al}$ mole fractions are beneficial for high 2DEG densities and thus low 2DEG sheet resistances, but disadvantageous for obtaining good ohmic contacts. Generally, the 2DEG sheet resistance values have to be taken into account when comparing the ohmic contact resistance values from different studies directly.

Metals with very high work functions, such as $\mathrm{Ni}, \mathrm{Pt}, \mathrm{Au}, \mathrm{Re}$ and $\mathrm{Ir}$ (Table 2.2), are most commonly used for Schottky diodes on $\mathrm{GaN}$. Ni is a very commonly used 
metal for a rectifying contact on $\mathrm{GaN}$ and $\mathrm{AlGaN}$. It forms a barrier height of $\sim 0.84$ $1.11 \mathrm{eV}$ for $\mathrm{Al}$ mole fraction from 0 to 0.23 . Ideality factor varies from 1.12 to 1.37 [32]. In multilayer Schottky contact schemes $\mathrm{Ni}$ is often used as a first layer adjacent to GaN due to its superior adhesion properties. In the study by Miura et al. [43], Ni/Pt/Au gave better Schottky contacts than either $\mathrm{Pt} / \mathrm{Au}$ or $\mathrm{Ni} / \mathrm{Au}$ on $\mathrm{Al}_{0.26} \mathrm{Ga}_{0.74} \mathrm{~N}$ and $\mathrm{n}$ GaN epilayers. Superior barrier heights of $1.47 \mathrm{eV}(\mathrm{n}-\mathrm{GaN})$ and $0.74 \mathrm{eV}(\mathrm{AlGaN})$ as well as the lowest reverse bias leakage currents were achieved. Ni/Pt/Au Schottky diodes were also compared to Ti/Ni/Pt/Au, $\mathrm{Pt} / \mathrm{Au}$ and Ti/Pt/Au by Miura et al. [44]. The Ni/Pt/Au scheme was found superior in both the barrier height and the reverse bias leakage, with $\phi_{B}=0.85 \mathrm{eV}$ and $J_{\text {leak }}=10^{-2} \mathrm{~A} / \mathrm{cm}^{2}$. Ti $/ \mathrm{Pt} / \mathrm{Au}$ scheme, on the other hand, displayed a very small variation in both leakage current and barrier height with RTA anneal temperatures, which typically range from 400 to $500^{\circ} \mathrm{C}$ [43].

Low barrier heights and high non-ideal leakage currents are still a problem in Schottky diodes to GaN and AlGaN materials. Some effort has been made in order to understand the mechanism of gate leakage current in AlGaN/GaN HFETs. One possible mechanism found in the paper by Hasegawa et al. [45] relies on a thin surface barrier model and assumes the presence of high density of deep donor defects (at $E^{C}-0.37 \mathrm{eV}$ ) near AlGaN surface, causing a narrowing of the Schottky barrier in such a way that electrons can tunnel through this barrier in both forward and reverse direction by means of the thermionic field-emission or the field-emission mechanism, depending on the temperature.

\subsubsection{Surface preparation, cleaning and chemical analysis}

Surface preparation plays an important role in any semiconductor material device fabrication, including that of GaN. In AlGaN/GaN heterostructures, surface states can directly degrade 2DEG density and ohmic contacts. According to Mishra's model, 
any impurities on the surface which act as acceptors or deep traps will directly reduce the 2DEG. The same impurities will counter-dope the semiconductor during the formation of an n-type tunnelling contact, which is a highly undesirable effect. The use of $\mathrm{Fe}, \mathrm{Be}, \mathrm{Zn}$ as well as Carbon (C) as acceptor-like point defects was previously demonstrated [46]. Thus particular attention has to be given to organic contamination. In addition, GaN surfaces have a very high chemisorption affinity for oxygen [39] and the presence of a thin insulating oxide layer can significantly impede the current flow across metal-semiconductor junctions.

The most common surface cleaning methods performed are chemical wet etches and plasma etches. Wet chemical treatments such as $\mathrm{HF}$, buffered $\mathrm{HF}, \mathrm{HCl}, \mathrm{HNO}_{3}$, $\mathrm{H}_{2} \mathrm{SO}_{4}, \mathrm{KOH}, \mathrm{NaOH}, \mathrm{NH}_{4} \mathrm{OH},\left(\mathrm{NH}_{4}\right)_{2} \mathrm{~S}_{x}$ have been reported to reduce or modify the surface contamination [47]. Some of these substances such as $\mathrm{HF}, \mathrm{KOH}$ and $\mathrm{HCl}$ are found to remove native $\mathrm{Al}$ and $\mathrm{Ga}$ oxides layers formed on $\mathrm{AlGaN}[35,39]$. While $\mathrm{HCl}$ is more effective for removing oxides, HF is more effective for removing carbon and/or hydrocarbon contamination. Physical treatments, which enhance the device performance by lowering the surface $\mathrm{N}$-vacancies and improving the surface stoichiometry include $\mathrm{H}_{2}$-plasma, $\mathrm{UV} / \mathrm{O}_{3}, \mathrm{O}_{2}$-plasma, $\mathrm{N}_{2}$-plasma, $\mathrm{Cl}_{2}$ based inductively coupled plasma (ICP) etching and $\mathrm{Ar} / \mathrm{N}$ sputtering [47]. Most fabrication steps involve a series of surface cleaning steps to ensure the removal of contaminants from several contamination sources.

Contact angle measurement can serve as an indicator of foreign material present on the surface, while XPS and SIMS can provide detailed information on the molecular composition of the contaminants $[48,49]$.

Surface analysis by XPS is accomplished by irradiating the sample with monoenergetic soft x-rays and analyzing the energy of the ejected electrons. $\mathrm{Mg} \mathrm{K} \alpha(1253.6 \mathrm{eV})$ or $\mathrm{Al} \mathrm{K \alpha}(1486.6 \mathrm{eV}) \mathrm{x}$-rays are usually used. These photons have limited penetrating power in a solid, on the order of 1-10 $\mu \mathrm{m}$. They interact with atoms in the surface 
region, causing electrons to be emitted by the photoelectric effect. The emitted electrons have measured kinetic energies given by:

$$
\mathrm{KE}=h \nu-\mathrm{BE}-\phi_{s}
$$

where $h \nu$ is the energy of the photon, BE is the binding energy of the atomic orbital from which the electron originates and $\phi_{s}$ is the spectrometer work function. Because each element has a unique set of binding energies, XPS can be used to identify and determine the concentration of the elements in the surface. Variations in the elemental binding energies arise from the differences in the chemical potential and polarizability of compounds. These chemical shifts can be used to identify the chemical state of the materials being analyzed. Argon ion etching is commonly used to obtain information on composition as a function of depth [50].

XPS measurements taken at different take off angles can be used to determine the thickness of a thin film present on the surface of another material by the following equation [51]:

$$
d_{\text {film }}=\lambda_{\text {film }} \sin \alpha \ln \left[I_{\text {film }} /\left(\beta I_{\text {sub }}\right)+1\right]
$$

where $d_{\text {film }}$ is the thickness of the thin film, $\lambda_{\text {film }}$ is the photoelectron effective attenuation length in the thin film, $\alpha$ is the take off angle and $I_{\text {film }}$ is the XPS peak intensity from the film, $\beta$ is the film-to-substrate XPS photosensitivity ratio and $I_{\text {sub }}$ is the XPS peak intensity from the substrate. This technique is referred to as an Angular-Resolved XPS (AR-XPS).

In SIMS the sample surface is sputtered by an ion beam and the emitted secondary ions are analyzed by a mass spectrometer. In static SIMS the primary ion dose is kept low $\left(\sim 10^{12}\right.$ ions $\left./ \mathrm{cm}^{2}\right)$ to ensure that every ion hits a fresh surface. In dynamic SIMS systems multiple layers of atoms are removed in a typical rate of 0.5 to $5 \mathrm{~nm} / \mathrm{s}$. Most common ion sources for dynamic SIMS are $\mathrm{O}^{-}, \mathrm{O}_{2}^{+}$and $\mathrm{Cs}^{+}$ions. Oxygen 
leads to the formation of oxides from which the original surface material emerges as a positively charged ion. Caesium is an efficient electron donor and mainly negatively charged secondary ions are generated. Dynamic SIMS typically use a magnetic mass analyzer, and for static SIMS the Time of Flight technique is most suitable [52].

Contact angle measurement can provide valuable information about the contaminants and oxide films on the surface. Contact angle is the angle at which the liquid interface meets the semiconductor interface. When purified water is used for contact angle measurement, surfaces which repel water (hydrophobic) yield high contact angles and surfaces that attract water (hydrophilic) yield small contact angles. Surface oxides formed on GaN or AlGaN layer were found to produce low contact angles. Carbon contamination, on the other hand, makes surfaces more hydrophobic $[48,53]$.

\subsection{Literature Review on GaN and AlGaN/GaN Surface Treatment with Si Thin Films}

Ion implantation of $\mathrm{Si}$ as a donor dopant in order to improve the tunnelling ohmic contacts to $\mathrm{AlGaN}$ and $\mathrm{GaN}$ materials and the associated diffusion and activation properties have been already well studied $[4,11,13,54-57]$. However, due to the high temperature annealing required to activate the dopants $\left(1,100^{\circ} \mathrm{C}\right)$ and to remove the ion induced crystal damage $\left(1,300^{\circ} \mathrm{C}\right)$, a significant amount of attention has been given to alternative $\mathrm{Si}$ doping/incorporation techniques in III-N materials.

The use of $\mathrm{Si}$ on AlGaN/GaN epilayers in order to improve ohmic contacts or 2DEG densities can be classified into three categories. The first category of studies involves the deposition of Si-containing thin films on the $\mathrm{AlGaN} / \mathrm{GaN}$ or $\mathrm{GaN}$ surface, followed by high temperature anneals and subsequent film removal. These 
studies appear to conclude that solid state diffusion is responsible for the incorporation of significant amount of $\mathrm{Si}$ in the top epilayer. The second category includes the improvement of ohmic contacts with Si thin films deposited prior to metal evaporation and/or in between metal layers of ohmic stack. In this scheme Si takes a direct part in chemical reactions which occur during ohmic contact alloying. The third group of investigations involves improvement to 2DEG densities and/or surface passivation with Si films without intentional diffusion.

Pearton et al. [58] reported on substantial oxygen diffusion from $\mathrm{SiO}_{2}$ film in ptype $\mathrm{GaN}$ in 1999, demonstrating that solid phase oxygen diffusion in GaN can take place at fairly low temperatures. $\mathrm{SiO}_{2}$ layers were deposited on $\mathrm{p}$ - $\mathrm{GaN}$ by inductively coupled plasma chemical vapour deposition (ICP-CVD) using an ${ }^{17} \mathrm{O}$-enriched $\mathrm{O}_{2}$ precursor. The samples were then annealed at $500-900^{\circ} \mathrm{C}$ for 5 minutes and the $\mathrm{SiO}_{2}$ was removed. SIMS profiling appeared to show significant in-diffusion of ${ }^{17} \mathrm{O}$ into the GaN under these conditions, with an incorporation depth of $180 \mathrm{~nm}$ after the $900^{\circ} \mathrm{C} 5$ minutes anneal. The ${ }^{17} \mathrm{O}$ diffusion profiles indicated that the high dislocation density in the GaN strongly affected the effective penetration depth. Two diffusion fronts were identified and attributed to bulk diffusion and threading dislocation diffusion. The latter was assumed to dominate the diffusion process and the following diffusion coefficient and activation energy were obtained: $D_{o}=4.5 \times 10^{-12} \mathrm{~cm}^{2} \mathrm{~s}^{-1}$ and $E_{d a}=$ $0.23 \mathrm{eV}$.

Lin et al. [59] has reported on a substantial reduction to specific contact resistivity on undoped GaN material using $\mathrm{Ti} / \mathrm{Al} / \mathrm{Ni}(15 / 120 / 30 \mathrm{~nm})$ ohmic contact scheme using Si films. A $1.5 \mu \mathrm{m}$ GaN epilayer surface was cleaned in Buffered Oxide Etchant (BOE) for $5 \mathrm{~min}$. before evaporating Si. The sandwich structure consisted of a $50 \mathrm{~nm}$ thick Si metal layer deposited by e-beam evaporation system directly on the GaN, followed by a $100 \mathrm{~nm} \mathrm{SiO} 2$ cap layer deposited by photo-CVD. Samples were annealed in $\mathrm{N}_{2}$ ambient for 2 hours in temperatures ranging from 825 to $1,000^{\circ} \mathrm{C}$. After the heat 
treatment a chemical solution consisting of $\mathrm{HF}, \mathrm{HNO}_{3}$, and $\mathrm{CH}_{3} \mathrm{COOH}$ at a ratio of 6:20:7 was used to remove the $\mathrm{Si}$ and cap layers. SIMS profiling has shown a significant amount of Si in GaN epilayer: the depth of apparent diffusion of Si in the GaN films was found to be $150 \mathrm{~nm}$ after $1,000^{\circ} \mathrm{C}$ annealing temperature. No appropriate SIMS control samples were used in this work. The heat treatment decreased the specific contact resistivity; specifically the $\rho_{c}$ values observed decreased from $3 \times 10^{-5}$ to $5.6 \times 10^{-7} \Omega-\mathrm{cm}^{2}$. The temperatures used for the heat treatments appeared to be significantly lower than seemingly required for a substantial diffusion to occur.

In 2005 Ishida et al. [60] reported on the use of Si deposition to achieve one order of magnitude improvement to $\mathrm{Ti} / \mathrm{Al}$ ohmic contact specific resistivity on AlGaN/GaN material. The contact area was covered by Si film while the rest of the area was covered by $\mathrm{SiO}_{2}$ film, followed by the annealing in a nitrogen atmosphere. After removing the $\mathrm{Si}$ and $\mathrm{SiO}_{2}$ film, $\mathrm{Ti} / \mathrm{Al}$ ohmic electrodes were formed. The increase of the Si concentration near the surface area by the selective doping technique was apparently confirmed by SIMS, however, the appropriate control sample, which would have consisted of coated with Si AlGaN surface (with no heat treatment), was not used (Fig. 2.19). The 30 min. annealing appeared to increase the Si concentration in the AlGaN layer by one order of magnitude from $2 \times 10^{20} \mathrm{~cm}^{-3}$ without any selective doping to $3 \times 10^{21} \mathrm{~cm}^{-3}$ after the annealing. Si appeared to diffuse at least $10 \mathrm{~nm}$ into AIGaN. Correspondingly, the specific contact resistivity was significantly reduced from $1 \times 10^{-5} \Omega$ - $\mathrm{cm}^{2}$ to $1.2 \times 10^{-6} \Omega-\mathrm{cm}^{2}$. Although the information on anneal temperatures was not included in this paper, one might infer from the paper written by co-author Murata et.al. [61] that a $1,000{ }^{\circ} \mathrm{C} 30 \mathrm{~min}$ long anneal was used. Even then this temperature is lower than one would expect for a significant diffusion of Si into AlGaN.

In 2004 Pan et al. [62] reported on apparent Si diffusion in p-GaN. To perform the diffusion process, a $600 \mathrm{~nm}$ thick Si film was sputter deposited directly on the 


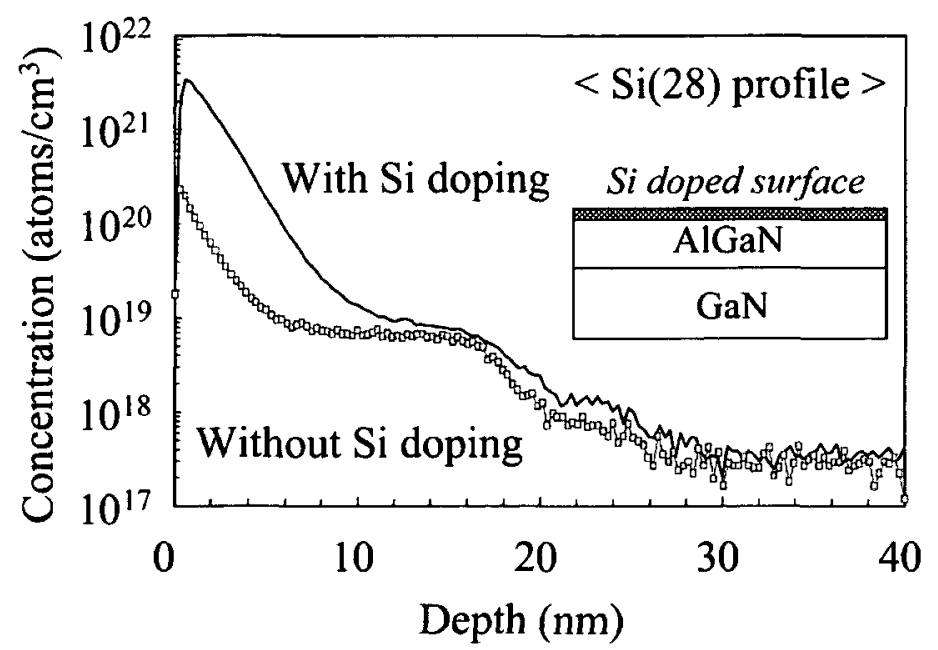

Figure 2.19: SIMS ${ }^{28} \mathrm{Si}$ profiles taken from a Si doped and from non-doped samples [60].

p-GaN layer, and a $200 \mathrm{~nm}$ thick $\mathrm{SiN}_{x}$ cap layer was deposited on top by PlasmaEnhanced Chemical Vapour Deposition (PECVD). Annealing was performed in flowing $\mathrm{N} 2$ atmosphere for 2 hours at temperatures from 800 to $1,100^{\circ} \mathrm{C}$. After annealing, the $\mathrm{SiN}_{x}$ encapsulant and the Si film were removed selectively from GaN using an $\mathrm{HF}: \mathrm{HNO}_{3}: \mathrm{CH}_{3} \mathrm{COOH}(6: 20: 7)$ solution. The ohmic metal stack consisted of $\mathrm{Ti} / \mathrm{Al}$ $(25 / 100 \mathrm{~nm})$. SIMS depth profiling revealed an apparent diffusion depth of 20 and $50 \mathrm{~nm}$ for 1,000 and $1,100^{\circ} \mathrm{C}$ respectively. The diffusion coefficient and the diffusion activation energies were derived to be $D_{o}=4 \times 10^{-7} \mathrm{~cm}^{2} \mathrm{~s}^{-1}$ and $E_{d a}=2.2 \mathrm{eV}$. Diffusion depths for 800 and $900^{\circ} \mathrm{C}$ were found to be 3 and $10 \mathrm{~nm}$ respectively. However, no control samples for SIMS measurements were included in this work.

In a study performed by Jang et al. [63] solid phase diffusion of Si in GaN was analyzed for $700-1,000^{\circ} \mathrm{C} 2$ hours long anneals and Si film thickness of $10-50 \mathrm{~nm}$ deposited by e-beam evaporation. Samples were encapsulated with $100 \mathrm{~nm}$ of $\mathrm{SiO}_{2}$ or $\mathrm{SiN}_{x}$ deposited by PECVD. After the anneals, BOE etch was used to remove the $\mathrm{SiO}_{2}$ and $\mathrm{HF} / \mathrm{HNO}_{3} / \mathrm{CH}_{3} \mathrm{COOH}$ wet etch to remove the Si. SIMS profiling revealed an apparent diffusion depths of $\sim 100 \mathrm{~nm}$ for $800-900^{\circ} \mathrm{C}$. The diffusion coefficient 
and the diffusion activation energies for Si diffusion were found to be $D_{o}=2.07 \times$ $10^{-4} \mathrm{~cm}^{2} \mathrm{~s}^{-1}$ and $E_{d a}=0.57 \mathrm{eV}$. The group concluded that the Si diffusion process lowered the specific contact resistivity of $\mathrm{Ti} / \mathrm{Al} / \mathrm{Pt} / \mathrm{Au}$ contacts by about a factor of 2 from $1.3 \times 10^{-4} \Omega-\mathrm{cm}^{2}$ to $7 \times 10^{-5} \Omega-\mathrm{cm}^{2}$. The final value of specific contact resistivity was not strongly dependent on temperature in the range $750-900^{\circ} \mathrm{C}$. Beyond a Si film thickness of $10 \mathrm{~nm}$, there was also no strong dependence of the contact properties. $\mathrm{SiO}_{2}$ capping was found to be superior to $\mathrm{SiN}_{x}$ and there was no surface roughening for anneal temperatures below $1,000^{\circ} \mathrm{C}$. This study lacks an appropriate control sample for SIMS as well.

Selective doping of source and drain regions with silicon oxynitride (SiON) thin film in AlGaN/GaN HEMTs was reported by Adivarahan and Khan et al. [64] in 2007. SiON layer was deposited using a PECVD system at a temperature of $200^{\circ} \mathrm{C}$ with silane, ammonia, nitrogen and oxygen as gas precursors and helium as a diluent. This was followed by $650^{\circ} \mathrm{C}$ anneal in a forming gas ambient for $5 \mathrm{~min}$. Significant increase in the Si concentration in the barrier and the 2-D channel region (around $20 \mathrm{~nm}$ below the surface) was observed from SIMS profiling. The ohmic contact scheme was not reported but ohmic contact resistance was improved form 0.55 Ohm$\mathrm{mm}$ to $0.23 \mathrm{Ohm}-\mathrm{mm}$. 2DEG sheet resistance was improved from $310 \mathrm{Ohm} / \mathrm{sq}$ to $260 \mathrm{Ohm} / \mathrm{sq}$. Given such a low anneal temperature and short anneal time, the solid phase diffusion of Si into AlGaN is very questionable in this work.

The same selective doping technique using SiON was reported by Deng and Khan et al. [65] in 2007. The information on the film thickness and techniques used to remove it were not included in these papers.

Kim et al. [66] investigated $\mathrm{Si} / \mathrm{Ti}$ ohmic contact to $\mathrm{n}-\mathrm{GaN}$ in 2000. Two schemes, $\operatorname{Ti}(15 \mathrm{~nm}) / \operatorname{Si}(50 \mathrm{~nm}) / \operatorname{Ti}(40 \mathrm{~nm}) / \mathrm{Au}(100 \mathrm{~nm})$ and $\operatorname{Ti}(15 \mathrm{~nm}) / \mathrm{Si}(146 \mathrm{~nm})-$ $/ \operatorname{Ti}(40 \mathrm{~nm}) / \mathrm{Au}(100 \mathrm{~nm})$, were employed in the study. Best specific contact resistivities of $3.5 \times 10^{-5} \Omega-\mathrm{cm}^{2}$ and $7.4 \times 10^{-6} \Omega-\mathrm{cm}^{2}$ were obtained after $900^{\circ} \mathrm{C}$ ohmic metal 
stepwise anneals for each scheme. Good ohmic contact resistivity was attributed to low work function of Ti-Si binary compounds: $\mathrm{Ti}_{5} \mathrm{Si}_{3}(3.69-3.73 \mathrm{eV})$, TiSi $(3.94-$ $3.99 \mathrm{eV})$ and $\mathrm{TiSi}_{2}(3.95-4.18 \mathrm{eV})$. It was also found that current conduction in the ohmic contact was occurring via thermionic emission over a low barrier height.

In a study performed by Desmaris et al. [67] the ohmic metal stack was comprised of $\mathrm{Si}(3 / 9 \mathrm{~nm}) / \mathrm{Ti} / \mathrm{Al} / \mathrm{Ni} / \mathrm{Au}$ were investigated using transmission electron microscopy and energy dispersive $\mathrm{x}$-ray spectroscopy. The dependence of the contact resistance on the Si layer thickness and the temperature was correlated to the microstructure of the alloyed contacts. The reduction of the contact resistance by inserting a $3 \mathrm{~nm}$ thick $\mathrm{Si}$ layer under the $\mathrm{Ti} / \mathrm{Al} / \mathrm{Ni} / \mathrm{Au}$ metallization was attributed to diffusion of the Si into the AlGaN layer. Increasing the Si thickness and/or the temperature resulted in the formation of Au-based silicides, which prevented the formation of low barrier TiN or AlN layers. Minimum ohmic contact resistance of $0.23 \mathrm{Ohm}-\mathrm{mm}$ was obtained for a $3 \mathrm{~nm}$ Si layer after $780^{\circ} \mathrm{C}$ anneal.

Mohammed et al. [68] reported on $\mathrm{Ti} / \mathrm{Si}(15 / 30 \mathrm{~nm}) / \mathrm{Al} / \mathrm{Si}(15 / 30 \mathrm{~nm}) / \mathrm{Mo} / \mathrm{Au}$ ohmic contact resistances to $\mathrm{AlGaN} / \mathrm{GaN}$ heterostructures in 2006. Excellent ohmic contact formation with contact resistance and specific contact resistivity as low as $0.12 \mathrm{Ohm}-\mathrm{mm}$ and $3.8 \times 10^{-7} \Omega-\mathrm{cm}^{2}$, respectively, have been obtained for $15 \mathrm{~nm} \mathrm{Si}$ thickness scheme. The processing window for annealing temperatures was increased in comparison to ohmic metal scheme with no Si.

In 2008 Onojima et al. [69] investigated the improvements to 2DEG densities and to $\mathrm{Ti} / \mathrm{Al} / \mathrm{Ni} / \mathrm{Au}$ ohmic contact resistances with the use of a $2 \mathrm{~nm}$ Si layer on AlGaN/GaN heterostructure surface. The starting material structure was comprised of $24.5 \mathrm{~nm}$ thick $\mathrm{Al}_{0.28} \mathrm{Ga}_{0.72} \mathrm{~N}$ barrier layer, 2,000 $\mathrm{nm}$ thick $\mathrm{GaN}$ layer and sapphire substrate. Si was deposited by e-beam evaporation. Ohmic contact resistance was improved from $0.96 \mathrm{Ohm}-\mathrm{mm}$ to $0.39 \mathrm{Ohm}-\mathrm{mm}$. After the Si deposition, the 2DEG 
density increased up to a level equivalent to that attained due to the Catalytic (Cat)CVD SiN deposition $\left(0.8 \times 10^{13} \rightarrow 1.2 \times 10^{13} \mathrm{~cm}^{-2}\right)$. There was no difference between the sample deposited with a Si layer alone and the sample deposited with a $\mathrm{Si} /$ Cat-CVD SiN stacked layer. This indicated that the increase in the 2DEG density can be induced by $\mathrm{Si}$ atom deposition as well as SiN passivation, and more importantly, this increase in density was closely related to the interface formation between Si and AlGaN. Hall mobility slightly decreased due to Si and Cat-CVD SiN deposition $\left(1.6 \times 10^{3} \rightarrow 1.4 \times 10^{3} \mathrm{~cm}^{2} /(\mathrm{Vs})\right)$. Resulting 2DEG sheet resistance improved from 480 to $380 \mathrm{Ohms} / \mathrm{sq}$. due to the Si deposition. AlGaN/GaN HFETs with a length of a T-shaped gate of $150 \mathrm{~nm}$ exhibited good pinch-off behavior, and the maximum drain current densities were $1.2 \mathrm{~A} / \mathrm{mm}$ for the device with Si treatment and $1.1 \mathrm{~A} / \mathrm{mm}$ for that without $\mathrm{Si}$. The $2 \mathrm{~nm}$ thick $\mathrm{Si}$ insert layer did not adversely affect the DC device characteristics. However, it can be seen from the drain DC $\mathrm{I}-\mathrm{V}$ curves provided in the paper that the pinch off voltage changed from $-11 \mathrm{~V}$ to $-13 \mathrm{~V}$ on Si-coated samples. Even more drastic 2DEG sheet resistance improvement was observed by Onojima et al. [70] in the case of $\operatorname{AlN}(2 \mathrm{~nm}) / \mathrm{GaN}$ structure (note that this is not a conventional AlGaN/GaN heterostructure) where resistance was remarkably reduced from 60,356 to $388 \mathrm{Ohm} / \mathrm{sq}$. by the deposition of a $2 \mathrm{~nm}$ thick Si layer on the AlN barrier surface. These results strongly support the "Si surface donor model", in which Si atoms located at the SiN/AlGaN interface act as positively ionized donors, which can partially neutralize the negative polarization charges of the AlGaN surface and thereby increase the 2DEG density (Fig. 2.20).

Surface passivation effects were studied on $\mathrm{AlGaN} / \mathrm{GaN}$ HEMTs using $\mathrm{SiO}_{2}$, $\mathrm{Si}_{3} \mathrm{~N}_{4}$, and SiON formed by PECVD by Arulkumaran et al. [71] in 2004. This study demonstrated that other Si-containing passivation schemes, in addition to the widely used $\mathrm{Si}_{3} \mathrm{~N}_{4}$, can reduce current collapse and increase transconductance and peak current density. 


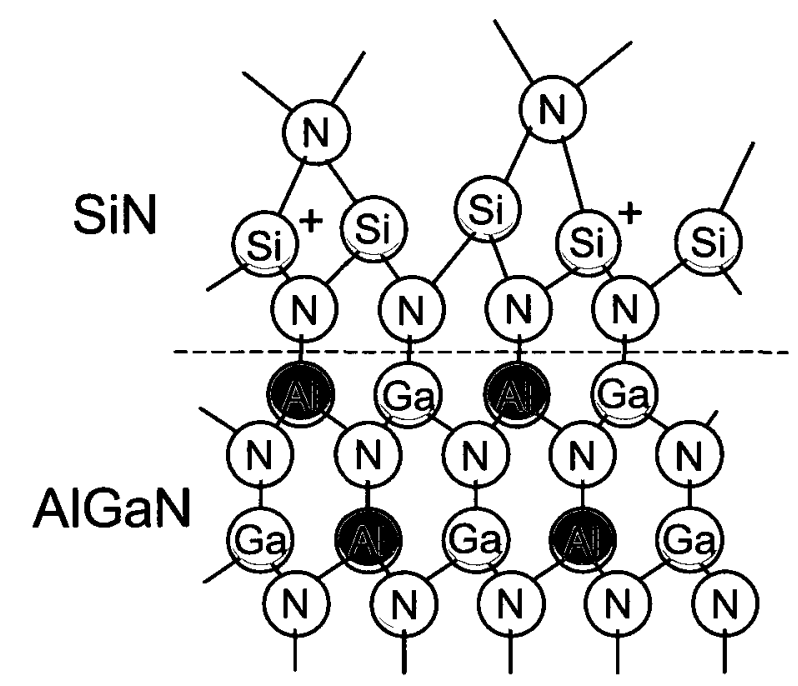

Figure 2.20: Schematic model of the atomic arrangement at the SiN/AlGaN interface [69].

The reviewed literature of GaN and $\mathrm{AlGaN} / \mathrm{GaN}$ surface treatment with Si thin films clearly demonstrates the importance of $\mathrm{Si}$ in $\mathrm{GaN}$ semiconductor materials. One of the greatest shortcomings of these studies, however, is the exclusion of other mechanisms by which the deposition of Si thin film, annealing and its removal can enhance the performance of ohmic contacts. Several other mechanisms, such as an incomplete Si film removal, chemical reaction on the topmost surface and roughening of the AlGaN layer can be responsible for changes in electrical performance of fabricated devices. Samples containing as-deposited Si films were not included in SIMS profiling, thus the results from these studies can include forward scattering of surface $\mathrm{Si}$ atoms by primary ions. This artifact will exaggerate the diffusion depth of Si. Incomplete Si removal durıng wet etches will result in apparent Si diffusion during SIMS profiling and can enhance ohmic contacts as was demonstrated by studies using Si inserts and Si-containing ohmic metal stacks.

Previous research does not provide an answer to questions such as whether deep diffusion of Si is necessary to achieve better electrical results or whether interaction between the Si thin film and the topmost $\mathrm{GaN}$ or AlGaN layer is sufficient. If high 
dislocation density is important for this technique, would it work on modern GaN material with lower dislocation densities?

Also, 2DEG sheet resistance was shown to improve after surface passivation with $\mathrm{Si}$ thin films, but 2DEG sheet resistance after Si etch and subsequent HEMT fabrication was not reported. All the studies involving the diffusion of Si from Si films to improve ohmic contacts used anneals in excess of 30 minute and it is not clear whether a shorter RTA anneal time would be sufficient to obtain similar results.

Lastly, the effect of Si treatments followed by Si film removal on AlGaN surface wetting properties has not been reported. Surface cleaning has a profound effect on fabricated device properties and thus a change in hydrophobic properties of AlGaN after Si thermal diffusion could potentially be the reason behind better ohmic contacts. 


\section{Chapter 3}

\section{DESIGN AND FABRICATION}

\subsection{Process Flow Considerations and Starting Ma- terial}

In order to study the effects and physical mechanism behind electrical enhancement in AlGaN/GaN devices after surface "treatments" and "coatings" with Si-containing thin films, three fabrication runs were developed and implemented. "Treatment" is defined as the e-beam deposition of Si-containing thin film, encapsulation with $\mathrm{SiO}_{2}$ deposited using a PECVD, RTA annealing and the films removal with a wet etch. "Coating" is defined as the e-beam deposition of Si film on the AlGaN/GaN surface. The three sets of GaN samples as well as the associated process runs were named "Batch-1", "Batch-2" and "Batch-3". The main goal of Batch-1 fabrication run was to confirm the changes in electrical performance on treated AlGaN/GaN surfaces with $\mathrm{Si}$ and SiON thin films. The 2DEG electron mobility and density were monitored during stepwise RTA annealing and the performance of fabricated test structures and devices was compared.

Batch-2 and Batch-3 fabrication runs built on the results from Batch-1 and also included samples permanently coated with Si film. The analysis and characterization 
of these samples were aided by SIMS profiling, XPS chemical composition and water contact angle measurements in order to investigate further the effect of Si treatments and coatings on the AlGaN surface.

Starting material for all three batches consisted of rectangular pieces diced from a high quality $\mathrm{Al}_{0.28} \mathrm{Ga}_{0.72} \mathrm{~N} / \mathrm{AlN} / \mathrm{GaN} / \mathrm{GaN}: \mathrm{Fe}(20 / 1 / 200 / 1,800 \mathrm{~nm})$ on SiC substrate wafer purchased from IQE corporation. Nominal sheet resistance $R_{s}$ for this passivated material is $\sim 300 \mathrm{Ohm} / \mathrm{sq}, 2 \mathrm{DEG}$ electron mobility $\mu$ is $2,000 \mathrm{~cm}^{2} /(\mathrm{Vs})$ and 2DEG sheet electron concentration $n_{s}$ is $1.0 \times 10^{13} \mathrm{~cm}^{-2}$. Each sample came from a different portion of the wafer thus some scatter in the results was expected between identically treated samples. The wafer structure and fabricated HEMT structure are shown together in Fig. 2.4.

Amorphous silicon (a-Si) was deposited with a Balzers BA510 e-beam evaporation system using a high resistivity $(1,500 \mathrm{Ohm}-\mathrm{cm})$ electronic grade $\mathrm{n}$-type (doped with phosphorous) Si wafer as a source. Silicon oxynitride was deposited in an Oxford PECVD system 100, using silane diluted in helium, ammonia, nitrous oxide and nitrogen. Silicon dioxide $\left(\mathrm{SiO}_{2}\right)$ was deposited with a Plasma-Therm 700 PECVD system using silane dilute in helium and nitrous oxide.

Existing experimental lithographic photo-masks were used to define device features using a Karl-Suss MJB-3 mask aligner with a $365 \mathrm{~nm} \mathrm{Hg}$ i-line source. The mask layout can be seen in Fig. 3.1. An Agilent 4155C semiconductor parameter analyzer and software controlled digital multimeter were used for electrical measurements of fabricated devices and test structures. HEMTs with the gate length of $1 \mu \mathrm{m}$, drain-to-gate spacing of $4 \mu \mathrm{m}$ and gate-to-source spacing of $1.5 \mu \mathrm{m}$ were used for electrical measurements in this work.

The following sections describe the development of process flows for each of these batches. A detailed step-by-step fabrication process flow for each run as well as an outline of NRC's baseline GaN technology can be found in Appendix A. 

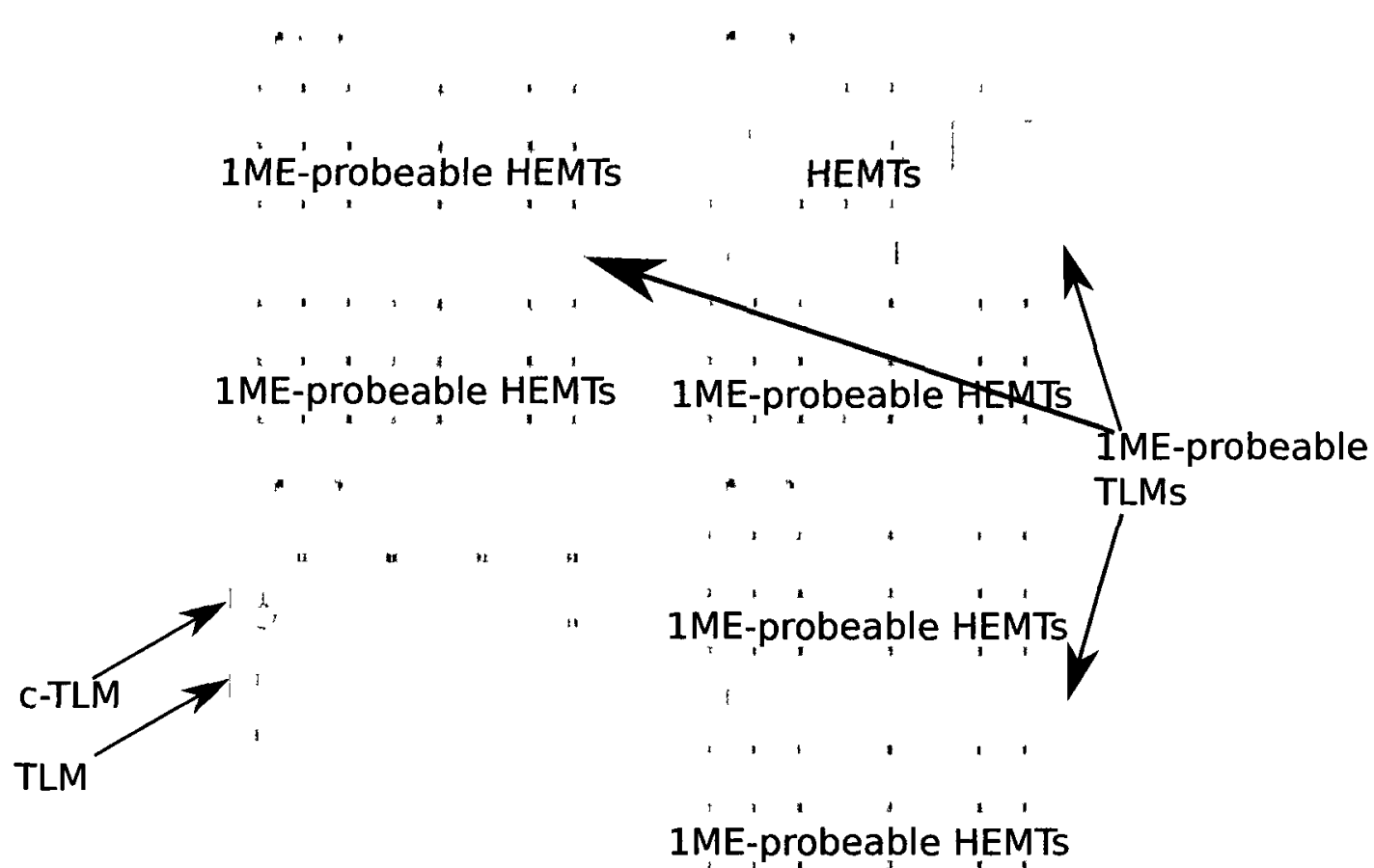

Figure 3.1: Lithographic photo-mask. TLM, c-TLM and HEMT structures are probeable after ohmic metal anneal.

\subsection{Batch-1 Process Flow Design and Measure- ments}

The focus of the first experimental run was to determine whether there were any observable electrical changes in 2DEG density and HEMT performance with Si surface treatments. The following two sources of Si were of most interest, since both of them were reported in the literature: $\mathrm{Si}$ and $\mathrm{SiON}$. The e-beam evaporation method for Si deposition was preferred, since the ohmic contact metal stack utilized the same deposition technique and future $\imath n$-situ deposition was possible. At the time of this work, however, it was not possible to evaporate both $\mathrm{Si}$ and ohmic contact metals in the same evaporation system due to equipment contamination concerns. Target samples were kept at low temperature during the evaporation to avoid crystallization 
and thereby obtain a-Si films [72]. However, it was expected that the a-Si would crystallize during subsequent high temperature anneals. Since neither the content of oxygen and nitrogen nor the refractive index of $\mathrm{SiON}$ thin film treatments has been reported in the literature, a 1:1 ratio of $\mathrm{SiO}_{2}$ to $\mathrm{Si}_{3} \mathrm{~N}_{4}$ was used as a starting point.

The a-Si thin film thickness was chosen to be $30 \mathrm{~nm}$, sufficiently thicker than the critical $10 \mathrm{~nm}$ thickness established by Jang et al. [63]. Magnets were used on corners of the samples to keep them in place during e-beam evaporation, thus the corners were free of a-Si. Since it was not clear whether SiON can contribute more or less $\mathrm{Si}$ atoms for possible diffusion than pure $\mathrm{Si}$, the silicon oxynitride thickness was set to be twice the value of a-Si: $60 \mathrm{~nm}$.

A $\mathrm{SiO}_{2}$ encapsulation layer was used by several groups in order to protect the AlGaN surface from degradation caused by high temperature anneals. It was found to be superior to the $\operatorname{SiN}_{x}$ encapsulation scheme by Jang et al. as well. Thus the encapsulation layer used here was comprised of $100 \mathrm{~nm} \mathrm{SiO}_{2}$, deposited by PECVD.

In order to establish whether high temperature annealing alone could produce observable electrical changes in $\mathrm{AlGaN} / \mathrm{GaN}$ heterostructures, RTA anneals were performed on two untreated samples. The samples were selectively stepwise RTA annealed in $\mathrm{N}_{2}$ flow starting at $400^{\circ} \mathrm{C}$ and ending at $950^{\circ} \mathrm{C}$ with Hall measurements of 2DEG density and electron mobility performed between each anneal. Anneal times ranged from 1 minute to 8 minutes for high and low anneal temperatures respectively. Samples coated with thin films had the corners wet etched in BOE for Hall system probes access. In order to reduce the error in Hall measurement, each sample was measured in four orientations achieved by 90 degree rotations. Hall measurement results provided a feedback on the requirement for further anneals for each sample.

Following the anneals, both the $\mathrm{SiO}_{2}$ cap and $\mathrm{SiON}$ were removed in $\mathrm{BOE}$. Etch times were determined using Si test wafers included in the PECVD deposition. $\mathrm{HF}: \mathrm{HNO}_{3}$ mixtures etch $\mathrm{Si}$ at rates exceeding $100 \mathrm{~nm} / \mathrm{s}$ for a wide range of 
compositions [73]. Therefore, Si films were etched from the Si-treated samples using $\mathrm{HF}: \mathrm{HNO}_{3}$ 1:3 mixture for 5-10 seconds.

Standard NRC processing steps were followed to implement ohmic contacts, isolation mesa, Schottky gates, silicon nitride passivation and metal-1 interconnects.

The following samples were used in this run:

- A1: a control sample.

- B1 and B2: samples RTA annealed to $850^{\circ} \mathrm{C}$ and $900^{\circ} \mathrm{C}$ respectively.

- S1 and S2: samples coated with a-Si, encapsulated with $\mathrm{SiO}_{2}, \mathrm{RTA}$ annealed to $950^{\circ} \mathrm{C}$ and $900^{\circ} \mathrm{C}$ respectively. The encapsulation and Si films were removed with the BOE and $\mathrm{HF}: \mathrm{HNO}_{3}$ etch.

- O1 and O2: samples coated with $\mathrm{SiON}$, encapsulated with $\mathrm{SiO}_{2}, \mathrm{RTA}$ annealed to $850^{\circ} \mathrm{C}$ and $900^{\circ} \mathrm{C}$ respectively. The encapsulation and SiON films were removed with the $\mathrm{BOE}$ etch.

The following electrical device measurements were performed after the specified fabrication steps:

- Ohmic Metal RTA Anneal: ohmic contact and sheet resistance from c-TLM structures.

- Gate metal anneal: ohmic contact and sheet resistance from c-TML and TLM structures, DC and pulsed HEMT I-V characteristics - current collapse, gate Schottky junction I-V characteristics, HEMT drain-source breakdown voltage.

- Passivation and Via1 etch: ohmic contact and sheet resistance from c-TML and TLM, DC and pulsed HEMT I-V characteristics - current collapse, gate Schottky junction characteristics, HEMT drain-source breakdown voltage.

- 1ME deposition: ohmic contact and sheet resistance from c-TML and TLM.

The following table (Table 3.1) provides the details on critical process steps as well as AlGaN/GaN material measurements for Batch-1. 


\begin{tabular}{|c|c|c|c|c|c|c|c|}
\hline Batch-1 Samples & A1 & B1 & B2 & S1 & S2 & O1 & $\mathrm{O} 2$ \\
\hline Hall Measurement(1) & $x$ & $\checkmark$ & $\checkmark$ & $\checkmark$ & $\checkmark$ & $\checkmark$ & $\checkmark$ \\
\hline E-beam a-Si $(30 \mathrm{~nm})$ & $x$ & $x$ & $x$ & $\checkmark$ & $\checkmark$ & $x$ & $x$ \\
\hline PECVD SiON (60 nm) & $x$ & $x$ & $x$ & $x$ & $x$ & $\checkmark$ & $\checkmark$ \\
\hline $\operatorname{PECVD~SiO}_{2}(100 \mathrm{~nm})$ & $x$ & $\times$ & $x$ & $\checkmark$ & $\checkmark$ & $\checkmark$ & $\checkmark$ \\
\hline Hall Measurement(2) & $x$ & $\checkmark$ & $\checkmark$ & $\checkmark$ & $\checkmark$ & $\checkmark$ & $\checkmark$ \\
\hline \multicolumn{8}{|l|}{ RTA Anneals } \\
\hline$-400^{\circ} \mathrm{C} 4 \mathrm{~min} / \mathrm{Hall}(3)$ & $x$ & $\checkmark$ & $\checkmark$ & $\checkmark$ & $\checkmark$ & $\checkmark$ & $\checkmark$ \\
\hline$-650^{\circ} \mathrm{C} \mathrm{I} 4 \mathrm{~min} / \mathrm{Hall}(4)$ & $x$ & $\checkmark$ & $\checkmark$ & $\checkmark$ & $\checkmark$ & $\checkmark$ & $\checkmark$ \\
\hline$-650^{\circ} \mathrm{C}$ II $4 \mathrm{~min} / \mathrm{Hall}(5)$ & $x$ & $\checkmark$ & $\checkmark$ & $\checkmark$ & $\checkmark$ & $\checkmark$ & $\checkmark$ \\
\hline$-700^{\circ} \mathrm{C} 8 \mathrm{~min} / \mathrm{Hall}(6)$ & $x$ & $\checkmark$ & $\checkmark$ & $\checkmark$ & $\checkmark$ & $\checkmark$ & $\checkmark$ \\
\hline$-750^{\circ} \mathrm{C} 2 \times 4 \mathrm{~min} / \mathrm{Hall}(7)$ & $x$ & $\checkmark$ & $\checkmark$ & $\checkmark$ & $\checkmark$ & $\checkmark$ & $\checkmark$ \\
\hline$-800^{\circ} \mathrm{C} 2 \times 2 \mathrm{~min} / \mathrm{Hall}(8)$ & $\times$ & $\checkmark$ & $\checkmark$ & $\checkmark$ & $\checkmark$ & $\checkmark$ & $\checkmark$ \\
\hline$-850^{\circ} \mathrm{C} 2 \times 1 \mathrm{~min} / \operatorname{Hall}(9)$ & $x$ & $\checkmark$ & $\checkmark$ & $\checkmark$ & $\checkmark$ & $\checkmark$ & $\checkmark$ \\
\hline$-875^{\circ} \mathrm{C} 2 \mathrm{x} 1 \mathrm{~min} / \mathrm{Hall}(10)$ & $x$ & $x$ & $\checkmark$ & $\times$ & $\checkmark$ & $x$ & $\checkmark$ \\
\hline$-900^{\circ} \mathrm{C} \mathrm{I} 2 \times 1 \mathrm{~min} / \mathrm{Hall}(11)$ & $x$ & $x$ & $\checkmark$ & $\times$ & $\checkmark$ & $x$ & $\checkmark$ \\
\hline$-900^{\circ} \mathrm{C}$ II $6 \times 1 \mathrm{~min} / \mathrm{Hall}(12)$ & $x$ & $x$ & $\checkmark$ & $\times$ & $\checkmark$ & $\times$ & $\checkmark$ \\
\hline$-925^{\circ} \mathrm{C} 1 \mathrm{~min} / \mathrm{Hall}(13)$ & $x$ & $x$ & $x$ & $\checkmark$ & $x$ & $x$ & $\times$ \\
\hline$-950^{\circ} \mathrm{C} 1 \mathrm{~min} / \mathrm{Hall}(14)$ & $\times$ & $x$ & $x$ & $\checkmark$ & $\times$ & $\times$ & $\times$ \\
\hline BOE Etch & $\times$ & $x$ & $x$ & $\checkmark$ & $\checkmark$ & $\checkmark$ & $\checkmark$ \\
\hline $\mathrm{HF}: \mathrm{HNO}_{3} 1: 35 \mathrm{sec}$ Etch & $\times$ & $x$ & $x$ & $\checkmark$ & $\checkmark$ & $x$ & $\times$ \\
\hline Hall Measurement(15) & $x$ & $\checkmark$ & $\checkmark$ & $\checkmark$ & $\checkmark$ & $\checkmark$ & $\checkmark$ \\
\hline Ohmic RTA $845^{\circ} \mathrm{C} 1 \mathrm{~min}$ & $\checkmark$ & $\checkmark$ & $\checkmark$ & $\checkmark$ & $\checkmark$ & $\checkmark$ & $\checkmark$ \\
\hline
\end{tabular}

Table 3.1: Batch-1 samples and the details on corresponding critical process steps and material measurements. The number in brackets indicates the Hall measurement label. 


\subsection{Batch-2 Process Flow Design and Measure- ments}

Batch-2 was designed to build on the results from the Batch-1 experiment. Due to promising results from the treatments with a-Si films, Batch-2 experiment focused on treatments from this source during one-time RTA anneals at temperatures of $850^{\circ} \mathrm{C}$ for 15 minutes, $950^{\circ} \mathrm{C}$ for 5 minutes and $1,000^{\circ} \mathrm{C}$ for 5 minutes. The same $30 \mathrm{~nm}$ e-beam evaporated a-Si and $100 \mathrm{~nm}$ PECVD deposited $\mathrm{SiO}_{2}$ films were used.

The question was raised whether the $\mathrm{HF}: \mathrm{HNO}_{3}$ wet etch alone could produce the electrical changes which were discovered on a-Si treated samples from Batch-1. Therefore, an additional sample was included with the purpose of determining the effect of this step on 2DEG densities (measured by Hall effect system before and after the wet etch) as well as on the performance of fabricated c-TLM/TLM structures and HEMTs.

In order to study the difference between Si-treated and Si-coated samples, two samples with $5 \mathrm{~nm}$ a-Si layer were included in the run. Reviewed literature indicated that the thickness of the coating layer should be around 2-3 nm. However, it was not possible to deposit $\mathrm{Si}$ in-situ prior to ohmic metal evaporation and thus native oxide formation which could reduce the thickness of the Si film was a concern. Si wafers stored for 1 week time period in air form a native oxide of 1.6 to $2 \mathrm{~nm}$ thick, weakly depending on initial surface cleaning [74]. Thus, in order to account for the native oxide growth, the a-Si thickness was chosen to be $5 \mathrm{~nm}$ deposited by e-beam evaporation.

For the investigation of the artifact produced during SIMS profiling as well as for XPS referencing, two additional samples with $5 \mathrm{~nm}$ of e-beam deposited a-Si were included.

It was anticipated that ohmic metal would require different alloying temperatures 
for each sample to reach optimum results, thus samples were selectively stepwise RTA annealed in $\mathrm{N}_{2}$ flow starting at $750^{\circ} \mathrm{C}$ and ending at $845^{\circ} \mathrm{C}$ with c-TLM measurements taken in between each anneal.

Ohmic metal got heavily oxidized due to a glitch in RTA system's control software. The ohmic contact resistance results from this batch became suspect. Further processing of this batch was halted following the ohmic metal RTA anneals. Batch-3 run was designed to replicate in parts the experiments planned to be performed on Batch-2 samples.

XPS (using Al K $\alpha 1486.6 \mathrm{eV}$ source) and dynamic SIMS (using Cs source) measurements were performed on reference and processed samples. Ar sputtering was employed for XPS measurement on the reference sample to reveal simultaneously the location of $\mathrm{Si}$ and $\mathrm{Ga}$ peaks where the former was used for charge referencing. Carbon peak referencing in XPS was shown to be unreliable for high temperature treated samples [75]. The elemental Si $2 p$ peak was assumed to have a location of $99.3 \mathrm{eV}$ to calibrate the energy axis. The "XPSPeak v4.1.1" software was used to fit the spectra in order to obtain the position and the intensity of each peak.

The following samples were used in this run:

- B1: a control sample.

- A1: $\mathrm{HF}: \mathrm{HNO}_{3}$ treated sample.

- S1 and S2: samples coated with a-Si, RTA annealed at $950^{\circ} \mathrm{C}$ for 5 min. The encapsulation and Si films were removed with the BOE and $\mathrm{HF}: \mathrm{HNO}_{3}$ etch.

- L1: a sample coated with a-Si, RTA annealed at $850^{\circ} \mathrm{C}$ for 15 min. The encapsulation and Si films were removed with the BOE and $\mathrm{HF}: \mathrm{HNO}_{3}$ etch.

- H1: a sample coated with a-Si, RTA annealed at $1,000^{\circ} \mathrm{C}$ for 5 min. The encapsulation and Si films were removed with the BOE and $\mathrm{HF}: \mathrm{HNO}_{3}$ etch.

- C1 and C2: samples coated with $5 \mathrm{~nm}$ of a-Si where the a-Si film stayed in place.

- R1 and R2: XPS and SIMS reference samples coated with $5 \mathrm{~nm}$ of a-Si. 
The following electrical device measurements were performed after specified fabrication steps:

- Ohmic Metal RTA Anneal: ohmic contact and sheet resistance from c-TLM, saturation current density over $2 \mu \mathrm{m}$ c-TLM gap.

Table 3.2 provides the details on critical process steps for Batch-2 as well as $\mathrm{AlGaN} / \mathrm{GaN}$ material measurements.

\begin{tabular}{|l|c|c|c|c|c|c|c|c|c|c|}
\hline Batch-2 Samples & B1 & A1 & S1 & S2 & L1 & H1 & C1 & C2 & R1 & R2 \\
\hline \hline E-beam a-Si (30 nm) & $\times$ & $\times$ & $\checkmark$ & $\checkmark$ & $\checkmark$ & $\checkmark$ & $\times$ & $\times$ & $\times$ & $\times$ \\
\hline E-beam a-Si (5 nm) & $\times$ & $\times$ & $\times$ & $\times$ & $\times$ & $\times$ & $\checkmark$ & $\checkmark$ & $\checkmark$ & $\checkmark$ \\
\hline PECVD SiO 2 (100 nm) & $\times$ & $\times$ & $\checkmark$ & $\checkmark$ & $\checkmark$ & $\checkmark$ & $\times$ & $\times$ & $\times$ & $\times$ \\
\hline Hall Measurement(1) & $\times$ & $\checkmark$ & $\times$ & $\times$ & $\times$ & $\times$ & $\times$ & $\times$ & $\times$ & $\times$ \\
\hline Thin Film RTA Anneals & & & & & & & & & & \\
\hline 850 C 15 min & $\times$ & $\times$ & $\times$ & $\times$ & $\checkmark$ & $\times$ & $\times$ & $\times$ & $\times$ & $\times$ \\
\hline -950 C 5 min & $\times$ & $\times$ & $\checkmark$ & $\checkmark$ & $\times$ & $\times$ & $\times$ & $\times$ & $\times$ & $\times$ \\
\hline -1,000 C 5 min & $\times$ & $\times$ & $\times$ & $\times$ & $\times$ & $\checkmark$ & $\times$ & $\times$ & $\times$ & $\times$ \\
\hline BOE Etch & $\times$ & $\times$ & $\checkmark$ & $\checkmark$ & $\checkmark$ & $\checkmark$ & $\times$ & $\times$ & $\times$ & $\times$ \\
\hline HF:HNO 1 1:3 10 sec & $\times$ & $\checkmark$ & $\checkmark$ & $\checkmark$ & $\checkmark$ & $\checkmark$ & $\times$ & $\times$ & $\times$ & $\times$ \\
\hline Hall Measurement(15) & $\times$ & $\checkmark$ & $\checkmark$ & $\checkmark$ & $\checkmark$ & $\checkmark$ & $\times$ & $\times$ & $\times$ & $\times$ \\
\hline Ohm. Cont. RTA Anneals & & & & & & & & & & \\
\hline -750/800/825/835 C 1 min & $\checkmark$ & $\checkmark$ & $\checkmark$ & $\checkmark$ & $\checkmark$ & $\checkmark$ & $\checkmark$ & $\checkmark$ & $\times$ & $\times$ \\
\hline -840 C 1 min & $\checkmark$ & $\checkmark$ & $\checkmark$ & $\checkmark$ & $\checkmark$ & $\checkmark$ & $\checkmark$ & $\checkmark$ & $\times$ & $\times$ \\
\hline -845 C 1 min & $\times$ & $\times$ & $\times$ & $\times$ & $\times$ & $\times$ & $\checkmark$ & $\checkmark$ & $\times$ & $\times$ \\
\hline SIMS Profiling & $\checkmark$ & $\times$ & $\checkmark$ & $\times$ & $\checkmark$ & $\checkmark$ & $\times$ & $\times$ & $\checkmark$ & $\times$ \\
\hline XPS Surface Measurement & $\times$ & $\checkmark$ & $\checkmark$ & $\checkmark$ & $\times$ & $\times$ & $\times$ & $\times$ & $\times$ & $\checkmark$ \\
\hline XPS with Ar Sputtering & $\times$ & $\times$ & $\times$ & $\times$ & $\times$ & $\times$ & $\times$ & $\times$ & $\times$ & $\checkmark$ \\
\hline
\end{tabular}

Table 3.2: Batch-2 samples and the details on corresponding critical process steps and material measurements. The number in brackets indicates the Hall measurement label. 


\subsection{Batch-3 Process Flow Design and Measure- ments}

Batch-3 was designed to replicate the Batch-2 experiment with additional XPS as well as water contact angle measurements. Two samples were dedicated to each category: control, Si-treated, and Si-coated samples.

The treated samples were coated with a $30 \mathrm{~nm}$ e-beam evaporated a-Si thin films and encapsulated with a $100 \mathrm{~nm}$ PECVD deposited $\mathrm{SiO}_{2}$. Both samples were RTA annealed at $950^{\circ} \mathrm{C}$ for $5 \mathrm{~min}$, followed by $\mathrm{BOE}$ and $\mathrm{HF}: \mathrm{HNO}_{3}$ etch. The a-Si film thickness of $5 \mathrm{~nm}$ was used for the coated samples. XPS surface chemical composition measurement was performed on Si-treated samples.

In order to establish and compare the difference in hydrophobic properties of the AlGaN surface, contact angle measurements were performed after each processing step leading up to ohmic metal deposition, such as the developing of photo-resist and final cleans (these steps had to be repeated prior to the actual ohmic metal deposition). This was followed by AR-XPS surface chemical analysis measurement at take off angles of 30,45 and 77.5 degrees in order to establish the thickness and the composition of the remnant Si-containing film.

After ohmic metal deposition, the samples were selectively stepwise RTA annealed in $\mathrm{N}_{2}$ flow starting at $825^{\circ} \mathrm{C}$ and ending at $835^{\circ} \mathrm{C}$ with c-TLM measurements performed in between.

Standard NRC processing steps were followed to implement an isolation mesa, Schottky gates, silicon nitride passivation and metal-1 interconnects.

The following samples were used in this run:

- B1 and B2: control samples.

- S1 and S2: samples coated with a-Si, RTA annealed at $950^{\circ} \mathrm{C}$ for $5 \mathrm{~min}$. The encapsulation and Si films were removed with the BOE and $\mathrm{HF}: \mathrm{HNO}_{3}$ etch. 
- C1 and C2: samples coated with $5 \mathrm{~nm}$ of a-Si where the a-Si film stayed in place.

The following electrical device measurements were performed after specified fabrication steps.

- Ohmic Metal RTA Anneal: ohmic contact and sheet resistance from c-TLM, saturation current density over $2 \mu \mathrm{m}$ c-TLM gap.

- Gate metal anneal: ohmic contact and sheet resistance from TLM, saturation current density over $2 \mu \mathrm{m}$ c-TLM gap, DC and pulsed HEMT I-V characteristics - current collapse, HEMT transconductance and threshold voltage.

- 1ME deposition: ohmic contact and sheet resistance from c-TML and TLM, saturation current densities over $2 \mu \mathrm{m}$ c-TLM gap, DC and pulsed HEMT I$\mathrm{V}$ characteristics - current collapse, gate Schottky junction I-V characteristics, HEMT transconductance and threshold voltage, gate leakage current versus drain voltage for standard and field plate assisted HEMTs.

Table 3.3 provides the details on critical process steps for Batch-3. 


\begin{tabular}{|c|c|c|c|c|c|c|}
\hline Batch-3 Samples & B1 & B2 & $\mathbf{S 1}$ & $\mathrm{S} 2$ & C1 & C2 \\
\hline E-beam a-Si $(30 \mathrm{~nm})$ & $\times$ & $x$ & $\checkmark$ & $\checkmark$ & $\times$ & $x$ \\
\hline E-beam a-Si $(5 \mathrm{~nm})$ & $x$ & $x$ & $x$ & $x$ & $\checkmark$ & $\checkmark$ \\
\hline $\mathrm{PECVD} \mathrm{SiO}_{2}(100 \mathrm{~nm})$ & $\times$ & $x$ & $\checkmark$ & $\checkmark$ & $x$ & $x$ \\
\hline \multicolumn{7}{|l|}{ Thin Film RTA Anneals } \\
\hline$-950^{\circ} \mathrm{C} 5 \mathrm{~min}$ & $\times$ & $x$ & $\checkmark$ & $\checkmark$ & $x$ & $x$ \\
\hline BOE Etch & $\times$ & $x$ & $\checkmark$ & $\checkmark$ & $\times$ & $x$ \\
\hline $\mathrm{HF}: \mathrm{HNO}_{3} 1: 310 \mathrm{sec}$ & $x$ & $x$ & $\checkmark$ & $\checkmark$ & $x$ & $x$ \\
\hline XPS Surface Measurement & $\times$ & $x$ & $\checkmark$ & $\checkmark$ & $\times$ & $\times$ \\
\hline \multicolumn{7}{|l|}{ Contact Angle Measurement } \\
\hline -Initial & $\checkmark$ & $\checkmark$ & $\checkmark$ & $\checkmark$ & $\checkmark$ & $\checkmark$ \\
\hline -Ace/IPA/DI & $\checkmark$ & $\checkmark$ & $\checkmark$ & $\checkmark$ & $\checkmark$ & $\checkmark$ \\
\hline —Ace/IPA Scrub/DI & $\checkmark$ & $\checkmark$ & $\checkmark$ & $\checkmark$ & $\checkmark$ & $\checkmark$ \\
\hline -HMDS Prime & $\checkmark$ & $\checkmark$ & $\checkmark$ & $\checkmark$ & $\times$ & $\checkmark$ \\
\hline -Resist Coat/Bake/Develop & $\checkmark$ & $\checkmark$ & $\checkmark$ & $\checkmark$ & $\times$ & $\checkmark$ \\
\hline -Microwave Descum & $\checkmark$ & $\checkmark$ & $\checkmark$ & $\checkmark$ & $x$ & $\checkmark$ \\
\hline -Ohmic Pre Clean/DI Rinse & $\checkmark$ & $\checkmark$ & $\checkmark$ & $\checkmark$ & $\times$ & $\checkmark$ \\
\hline _2nd DI Rinse & $\checkmark$ & $\checkmark$ & $\checkmark$ & $\checkmark$ & $x$ & $\checkmark$ \\
\hline-20 hours storage & $\checkmark$ & $\checkmark$ & $\checkmark$ & $\checkmark$ & $\times$ & $\checkmark$ \\
\hline AR-XPS $30^{\circ} / 45^{\circ} / 77.5^{\circ}$ & $x$ & $x$ & $x$ & $\checkmark$ & $x$ & $x$ \\
\hline \multicolumn{7}{|l|}{ Ohm. Cont. RTA Anneals } \\
\hline$-825 / 830^{\circ} \mathrm{C} 1 \mathrm{~min}$ & $\checkmark$ & $\checkmark$ & $\checkmark$ & $\checkmark$ & $\checkmark$ & $\checkmark$ \\
\hline$-835^{\circ} \mathrm{C} 1 \mathrm{~min}$ & $\times$ & $\times$ & $\times$ & $\checkmark$ & $\checkmark$ & $\times$ \\
\hline
\end{tabular}

Table 3.3: Batch-3 samples and the details on corresponding critical process steps and material measurements. 


\section{Chapter 4}

\section{RESULTS}

\subsection{Introduction}

The results of the electrical and chemical measurements are presented in this chapter. Since the main goal of Si treatments and coatings was to improve the electrical performance of fabricated devices and because the electrical measurements for the most part were performed prior to the the surface composition measurements, they are presented in the beginning of the chapter. The 2DEG properties measured by Hall effect are given first, followed by the electrical measurement results of fabricated TLM structures and transistors. The chapter is concluded with the most important and telling results obtained from the surface chemical composition measurements obtained by SIMS and XPS as well as with the water contact angle measurement on the AlGaN surface. Control samples processed by conventional means are marked with a ' $f$ ' throughout this chapter for easier identification. 


\subsection{DEG Sheet Resistance, Electron Density and Mobility by Hall}

\subsubsection{Batch-1}

The results of Hall measurements performed on Batch-1 samples are organized in three groups of plots. Fig. 4.1 shows the evolution of the 2DEG sheet resistance as a function of processing and RTA anneal steps (Table 3.1). Fig. 4.2 and Fig. 4.3 present the evolution of 2DEG electron mobility and density.

There is a clear advantageous trend in $\mu, n_{s}$ and thus $R_{s}$ on Si-treated samples $\mathrm{S} 1$ and S2 before Si etch. Following the removal of the films, S1 and S2 displayed an increase in the 2DEG carrier mobility and a decrease in the 2DEG sheet carrier density. This resulted in a modest increase in $R_{s}$, however, the final value remained very low, 310 and $320 \mathrm{Ohm} / \mathrm{sq}$ for samples S1 and S2 respectively. The final mobilities on samples S1, S2, O1 and O2 are comparable and are better than on RTA annealed samples B1 and B2. 


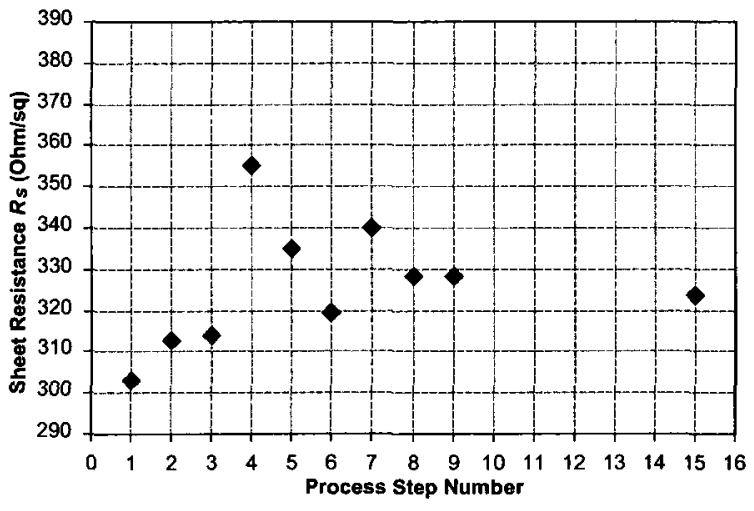

(a) B1, RTA annealed control

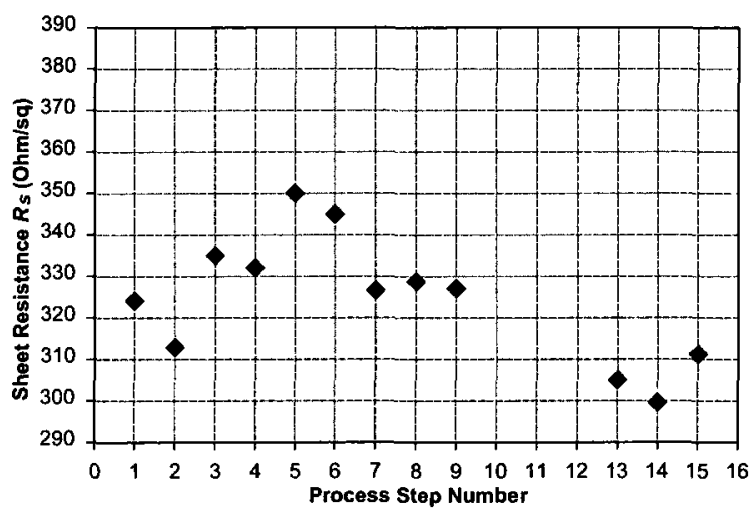

(c) S1, a-Si film

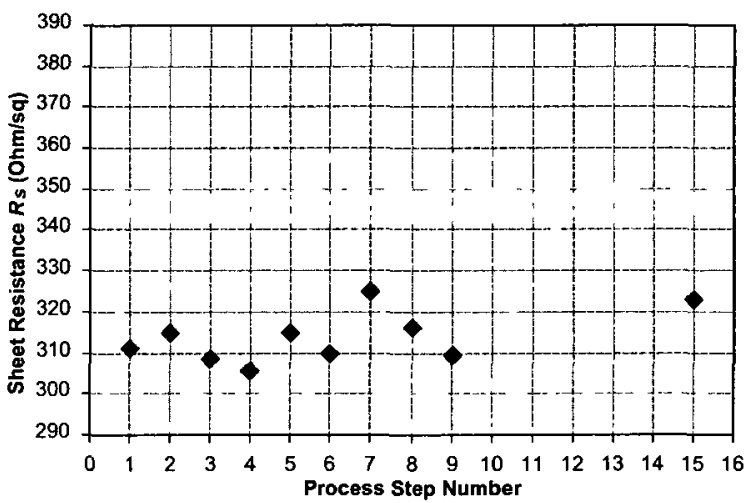

(e) O1, SiON film

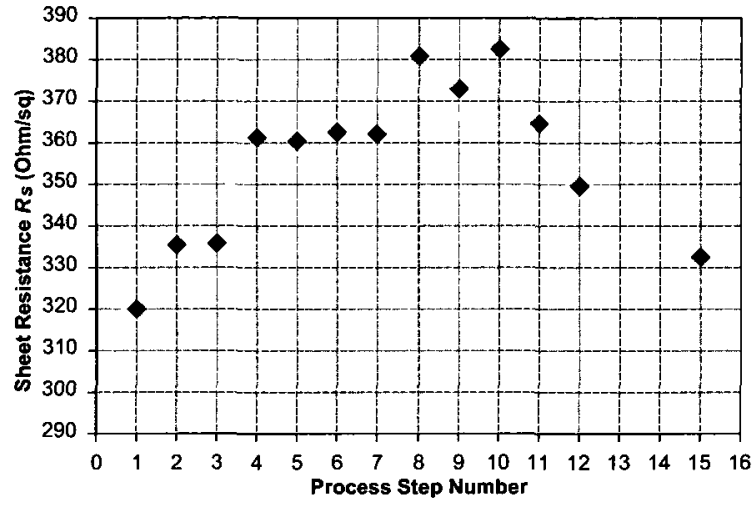

(b) B2, RTA annealed control

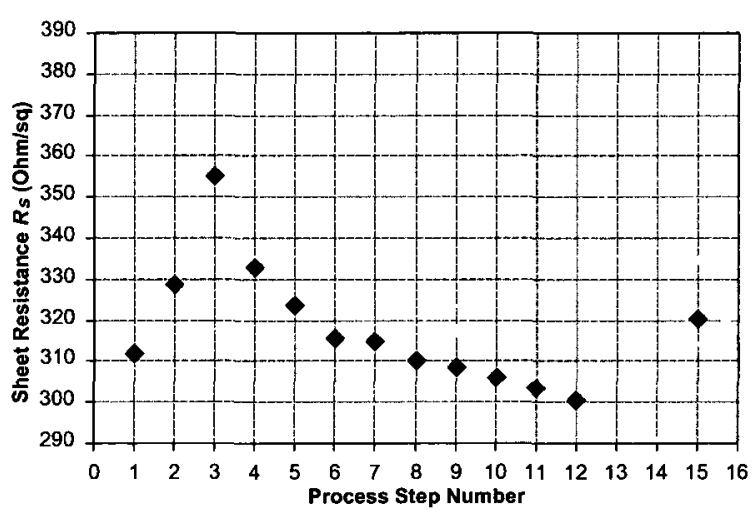

(d) S2, a-Si film

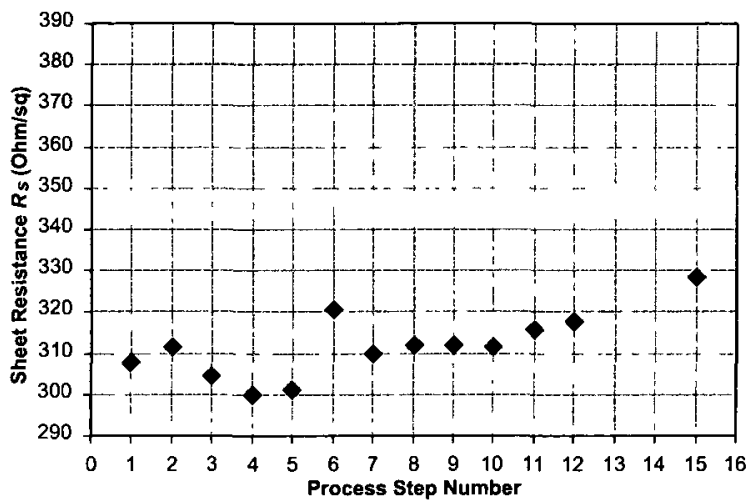

(f) $\mathrm{O} 2, \mathrm{SiON}$ film

Figure 4.1: Batch-1 evolution of the 2DEG sheet resistance $R_{s}$ as a function of processing steps. The spread associated with each data point is $\sim 5-10 \mathrm{Ohm} / \mathrm{sq}$. Step 1 and 2: before and after the deposition of the thin films. Steps 3-14: RTA anneals. Step 15: after the $\mathrm{HF}: \mathrm{HNO}_{3}$ wet etch. 


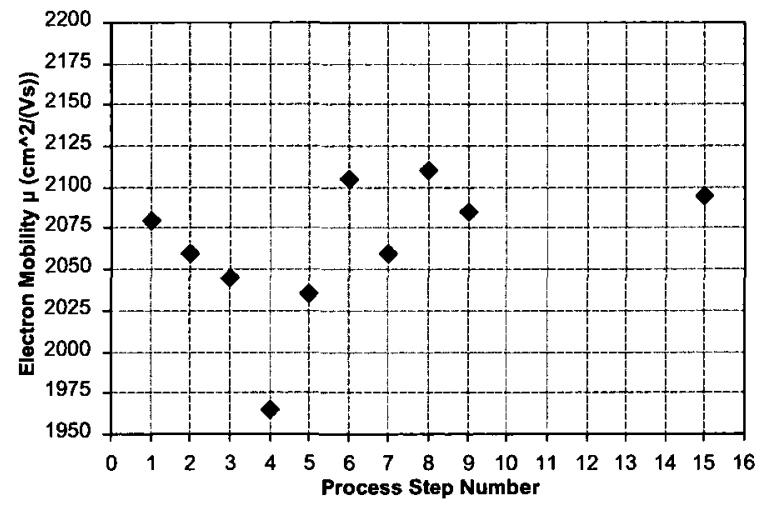

(a) B1, RTA annealed control

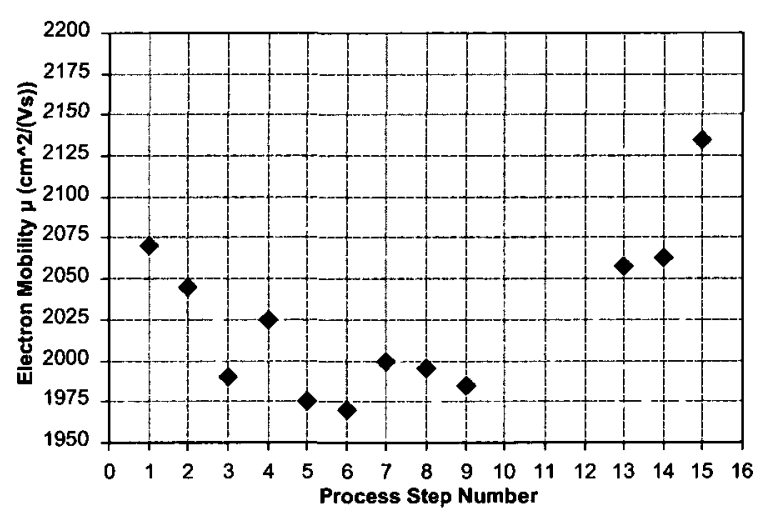

(c) S1, a-Si film

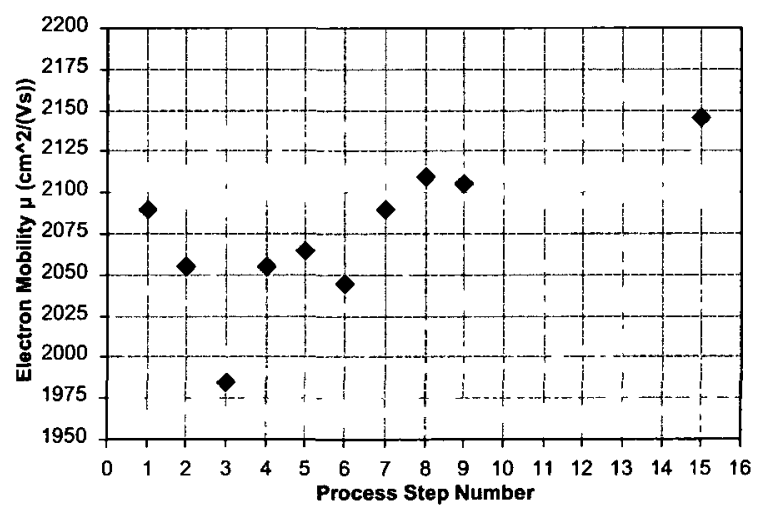

(e) O1, SiON film

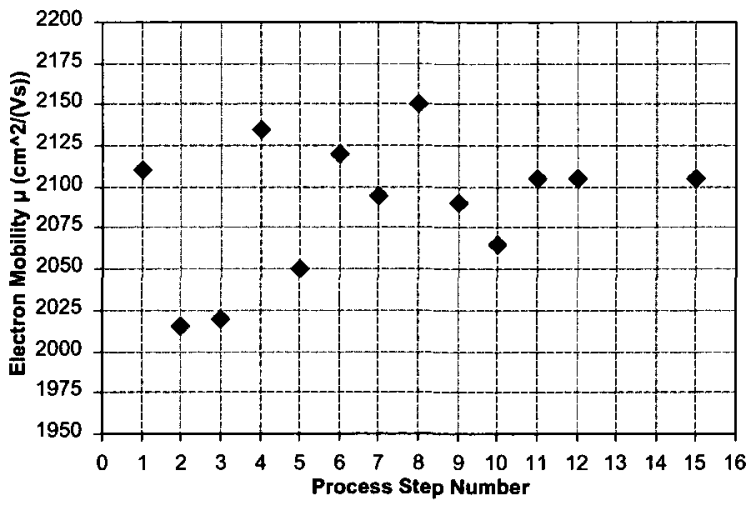

(b) B2, RTA annealed control

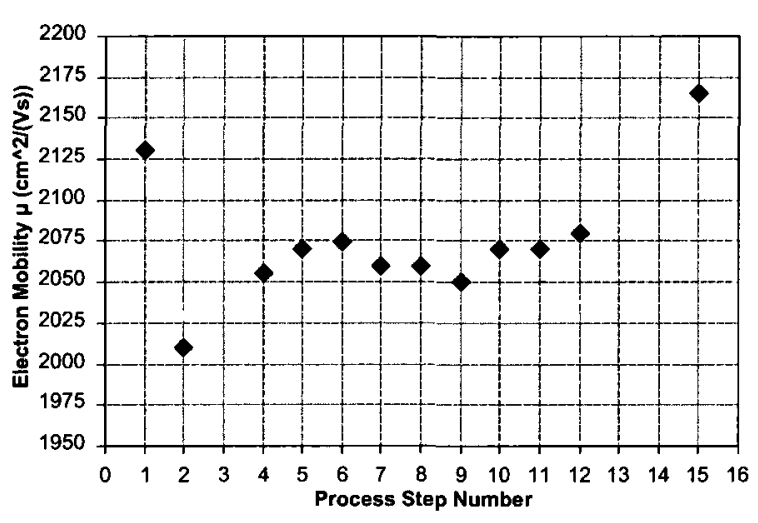

(d) S2, a-Si film

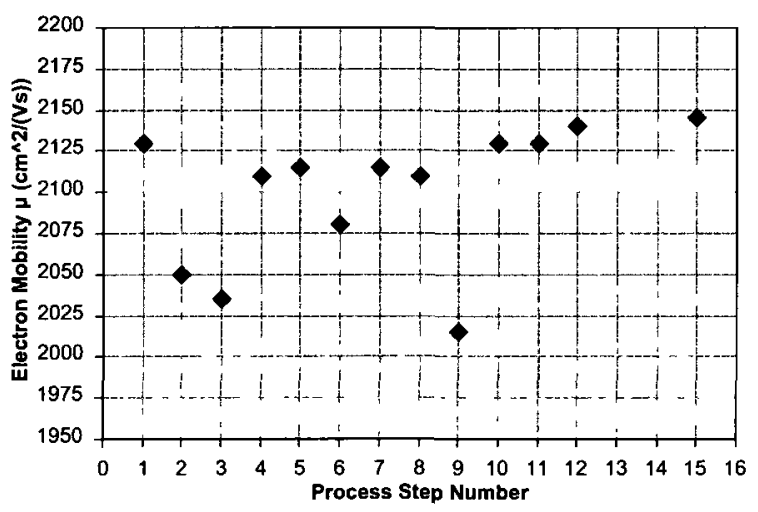

(f) O2, SiON film

Figure 4.2: Batch-1 evolution of the 2DEG electron mobility $\mu$ as a function of processing steps. Step 1 and 2: before and after the deposition of the thin films. Steps 3-14: RTA anneals. Step 15: after the $\mathrm{HF}: \mathrm{HNO}_{3}$ wet etch. 


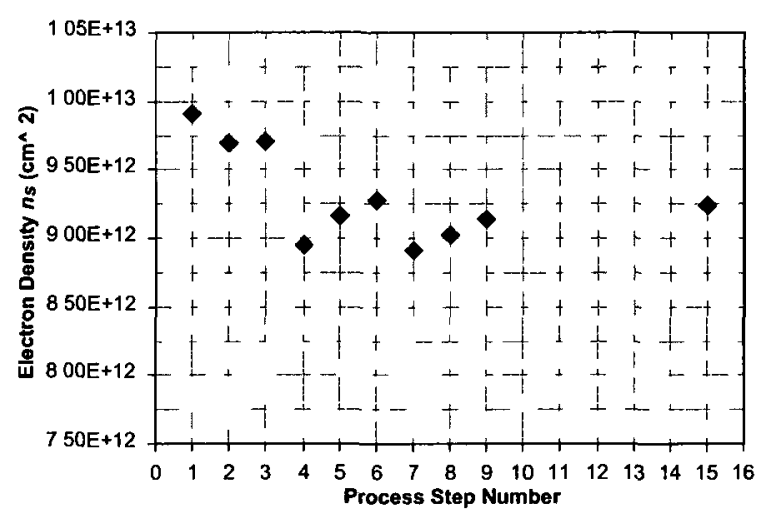

(a) B1, RTA annealed control

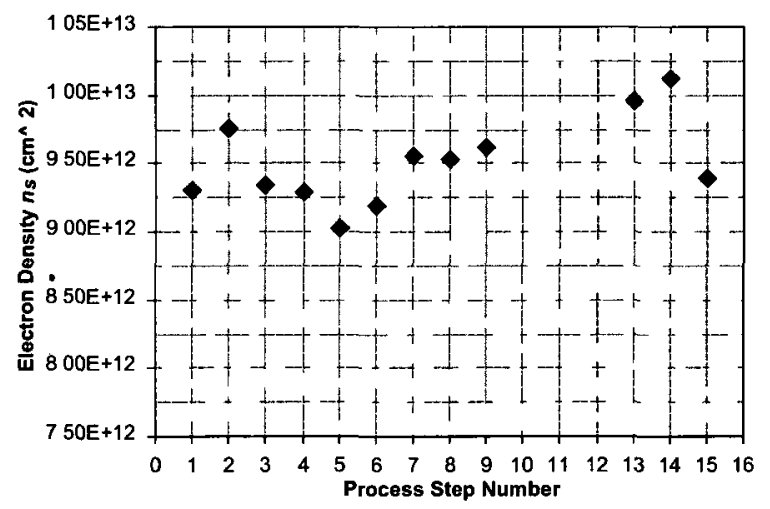

(c) S1, a-Sı film

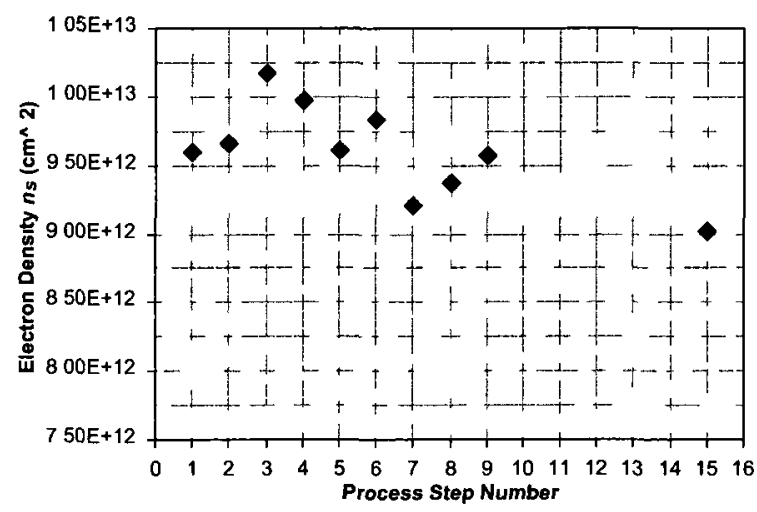

(e) $\mathrm{O} 1, \mathrm{SION}$ film

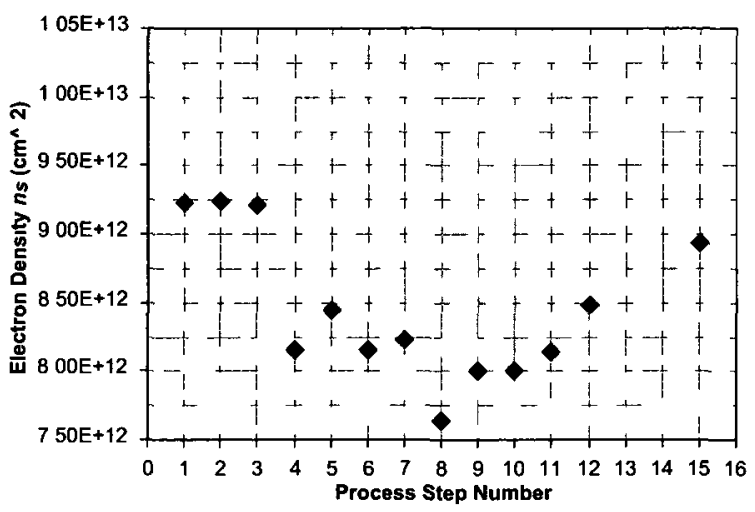

(b) B2, RTA annealed control

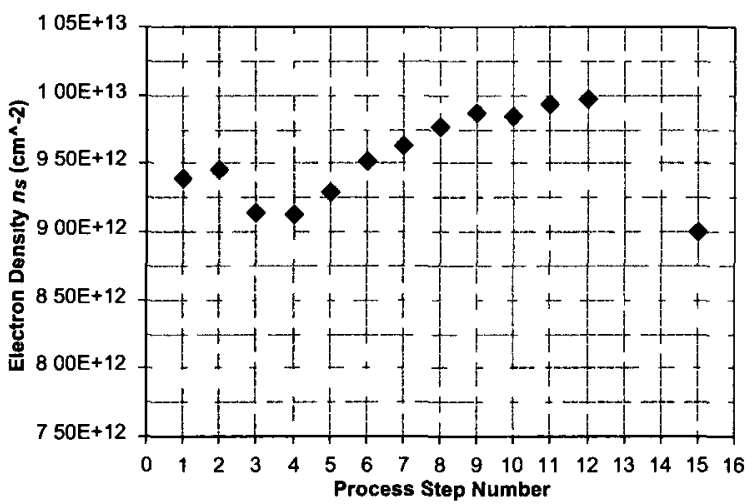

(d) S2, a-Si film

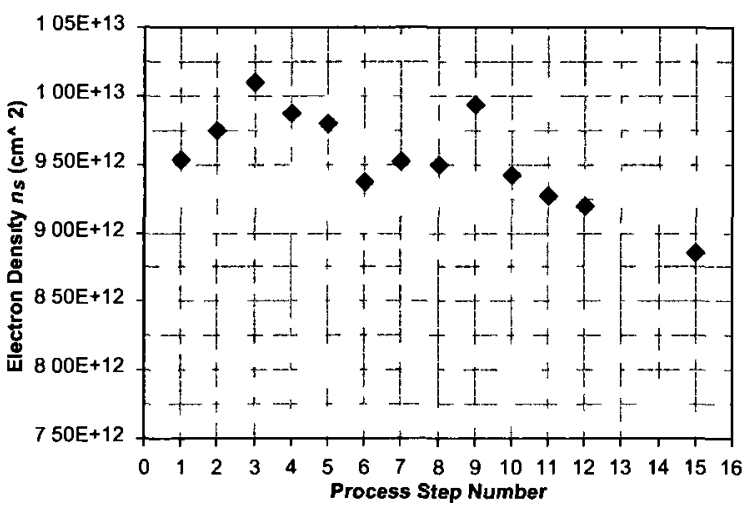

(f) $\mathrm{O} 2, \mathrm{~S} \mathrm{ON}$ film

Figure 4.3: Batch-1 evolution of the 2DEG electron density $n_{s}$ as a function of processing steps Step 1 and 2 before and after the deposition of the thin

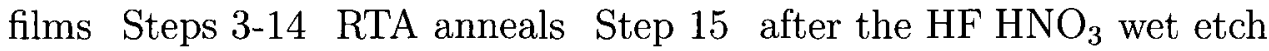




\subsubsection{Batch-2}

Table 4.1 summarizes the Hall system measurements of Batch-2 samples. An improvement to the 2DEG sheet resistance was seen on sample A1 following the $\mathrm{HF}: \mathrm{HNO}_{3}$ wet etch. However, this improvement did not materialize into better sheet resistances in TLM structures, as shown further in this chapter. This result was not unexpected, since unpassivated AlGaN/GaN surfaces are very sensitive to surface conditions. Very low sheet resistances were obtained on all samples except S1.

\begin{tabular}{|c|c|c|c|c|c|c|}
\hline & \multicolumn{3}{|c|}{ Before the Thin Film Dep. } & \multicolumn{3}{|c|}{ After $\mathrm{HF}: \mathrm{HNO}_{3}$ Wet Etch } \\
\hline & $R_{s}$ & $\mu$ & $n_{s}$ & $R_{s}$ & $\mu$ & $n_{s}$ \\
\hline & $\mathrm{Ohm} / \mathrm{sq}$ & $\mathrm{cm}^{2} /(\mathrm{Vs})$ & $\mathrm{cm}^{-2}$ & $\mathrm{Ohm} / \mathrm{sq}$ & $\mathrm{cm}^{2} /(\mathrm{Vs})$ & $\mathrm{cm}^{-2}$ \\
\hline $\mathrm{A} 1$ & 326 & 2020 & $9.49 \times 10^{12}$ & 311 & 2040 & $9.86 \times 10^{12}$ \\
\hline S1 & & & & 339 & 1950 & $9.42 \times 10^{12}$ \\
\hline $\mathrm{S} 2$ & & & & 300 & 2000 & $1.04 \times 10^{13}$ \\
\hline L1 & & & & 317 & 1950 & $1.01 \times 10^{13}$ \\
\hline $\mathrm{H} 1$ & & & & 302 & 1990 & $1.04 \times 10^{13}$ \\
\hline
\end{tabular}

Table 4.1: Batch-2 2DEG sheet resistance, electron mobility and density before the deposition of the a-Si thin film and after the $\mathrm{HF}: \mathrm{HNO}_{3}$ wet etch. 


\subsection{Ohmic Contacts and 2DEG Sheet Resistance from c-TLM and TLM Structures}

\subsubsection{Batch-1}

Table 4.2 summarizes Batch-1 TLM and c-TLM measurement results at several stages of the process flow. Superior to other samples ohmic contact and 2DEG sheet resistances were obtained on Si-treated samples S1 and S2. However, from the control sample measurements, as well as Batch-2 and Batch-3 annealing conditions, it was established that the ohmic metal in Batch-1 was over-annealed. Better 2DEG sheet resistance on Si-treated samples after the passivation step, which normally improves the 2DEG sheet resistance, may indicate that the Si treatments provide additive passivating effects. The variation between the c-TLM and TLM measurements represents the scatter of the data across the sample since the two patterns were located apart from each other. 


\begin{tabular}{|l|ccc|cc|cc|cc|}
\hline & \multicolumn{2}{|c|}{ RTA anneal } & \multicolumn{2}{|c|}{ Gate Anneal } & \multicolumn{2}{c|}{ Passivation } & \multicolumn{2}{c|}{ 1ME Dep. } \\
\cline { 2 - 10 } & \multicolumn{2}{|c|}{$R_{c}$} & $R_{s}$ & \multicolumn{2}{c|}{$R_{\mathrm{c}}$} & $R_{s}$ & $R_{c}$ & $R_{s}$ & \multicolumn{2}{c|}{$R_{c}$} & $R_{s}$ \\
\cline { 2 - 10 } & Ohm-mm & Ohm/sq & Ohm-mm & Ohm/sq & Ohm-mm & Ohm/sq & Ohm-mm & Ohm/sq \\
\hline \hline${ }^{\dagger}$ A1 c-TLM & 165 & 322 & - & - & 160 & 325 & 163 & 323 \\
${ }^{\dagger}$ A1 TLM & - & - & 186 & 319 & 180 & 314 & 168 & 314 \\
\hline B1 c-TLM & 112 & 437 & - & - & 112 & 313 & 107 & 300 \\
B1 TLM & - & - & 129 & 307 & 123 & 304 & 120 & 304 \\
\hline B2 c-TLM & 138 & 503 & - & - & 114 & 340 & 119 & 333 \\
B2 TLM & - & - & 145 & 323 & 139 & 320 & 129 & 320 \\
\hline S1 c-TLM & 085 & 360 & - & - & 084 & 293 & 083 & 283 \\
S1 TLM & - & - & 093 & 307 & 09 & 289 & 082 & 286 \\
\hline S2 c-TLM & 085 & 370 & - & - & 078 & 298 & 083 & 290 \\
S2 TLM & - & - & 102 & 317 & 093 & 291 & 084 & 290 \\
\hline O1 c-TLM & 130 & 463 & - & - & 124 & 316 & 128 & 309 \\
O1 TLM & - & - & 141 & 311 & 138 & 305 & 126 & 304 \\
\hline O2 c-TLM & 140 & 455 & - & - & - & - & - & - \\
O2 TLM & - & - & 144 & 314 & - & - & - & - \\
\hline
\end{tabular}

Table 4.2: Batch-1 ohmic contact and 2DEG sheet resistances from a four point probe measurement of c-TLM and TLM structures. Averages of two to four measurements were taken for each sample. 1ME TLM results combine the measurements of ohmic-probeable as well as of 100x64 and 100x32 $\mu \mathrm{m}$ 1MEprobeable TLMs.

Comparison bar plots of the final ohmic contact and 2DEG sheet resistances obtained after passivation and 1ME deposition step in Batch-1 are given in the figure below (Fig. 4.4). 


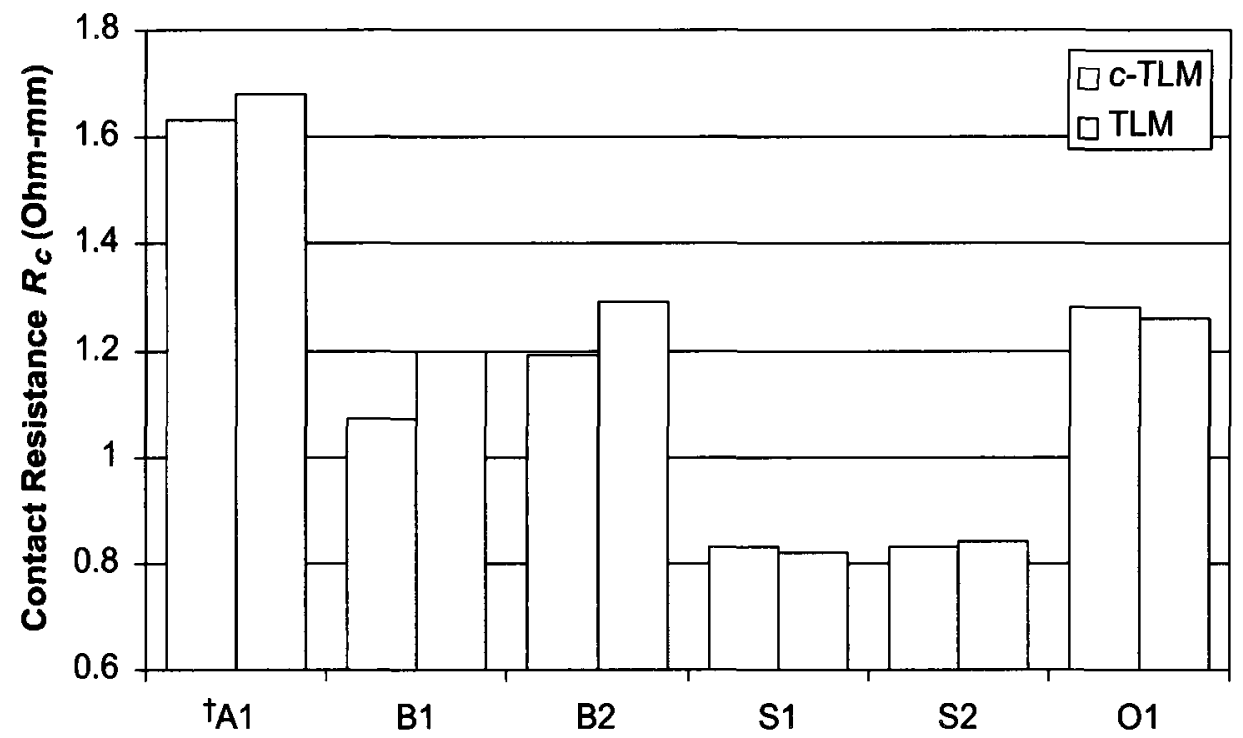

(a) Ohmic contact resistance $\left(\mathrm{R}_{c}\right)$ from c-TLM (red) and TLM (green)

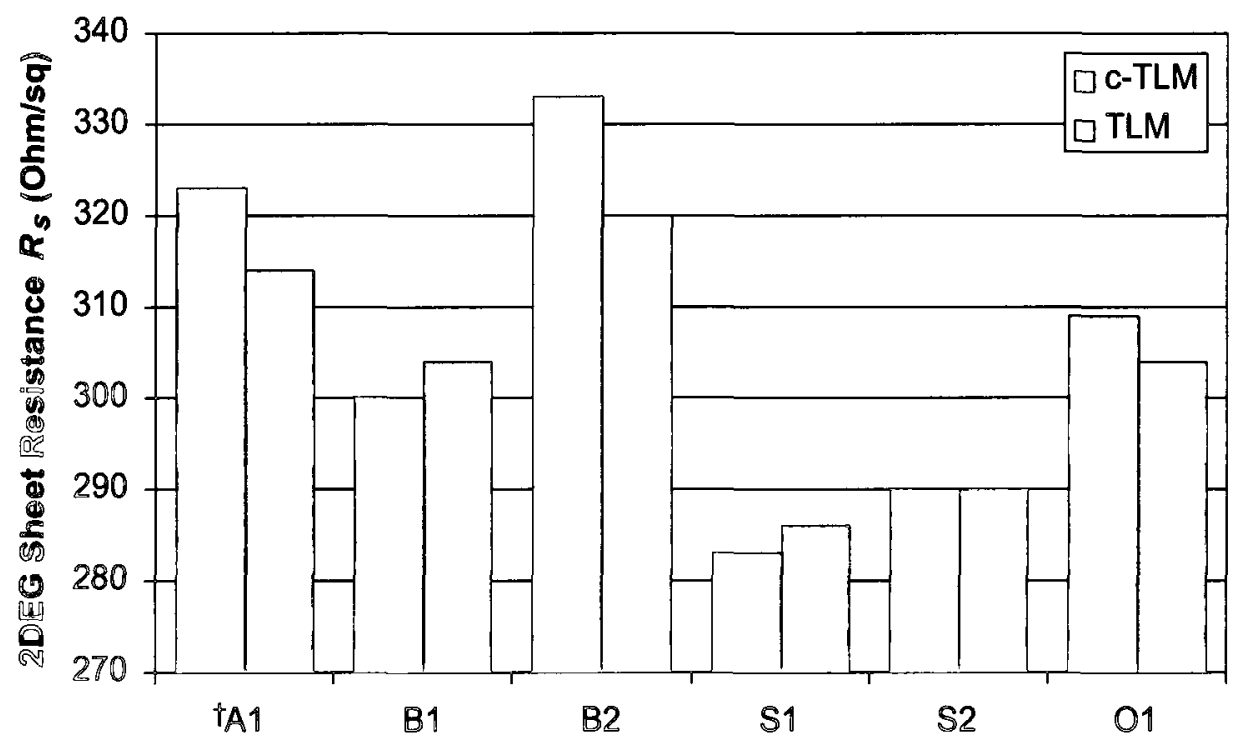

(b) $2 \mathrm{DEG}$ sheet resistance $\left(\mathbb{R}_{s}\right)$ from c-TLM (red) and TLM (green)

Figure 4.4: Batch-1 ohmic contact and 2DEG sheet resistances after passivation and $1 \mathrm{ME}$ deposition steps. 


\subsubsection{Batch-2}

Ohmic contact and 2DEG sheet resistances as well as the saturation current density over $2 \mu \mathrm{m}$ c-TLM gap at $9.9 \mathrm{~V}$ bias after RTA anneals at temperatures of 825 , 835,840 and $845^{\circ} \mathrm{C}$ are summarized in Table 4.3. Further processing of this batch was halted due to the heavy oxidation of the ohmic metal stack. The metal on the samples has become discoloured, however, the electrical probing could still be successfully performed. It should be noted that this accidental oxidation affected the ohmic metal, which is known to be easily oxidized. However, the temperature of $800^{\circ} \mathrm{C}$ and low oxygen content inside the RTA system were not sufficient to alter significantly the composition of treated, coated and untreated AlGaN layers. The electrical results, however, are questionable given the oxidation of the metal.

All Si-treated and Si-coated samples demonstrated far superior characteristics than the control sample ${ }^{\dagger} \mathrm{B} 1$ and the acid treated sample $\mathrm{A} 1$ under these circumstances. Optimum alloying temperatures for Si-treated samples (S1, S2, L1, H1) and for Si-coated samples $(\mathrm{C} 1, \mathrm{C} 2)$ were shifted to higher values. Si-treated and coated samples exhibited significantly higher saturation current densities over $2 \mu \mathrm{m}$ c-TLM gap, the measurement which relies both on the performance of the ohmic contacts and the sheet resistance. In addition, low $(<1 \mathrm{Ohm}-\mathrm{mm})$ contact resistance values were achieved more consistently on the treated and coated samples. It can be concluded that the Si treatments and coatings can improve the ohmic contacts and device current densities in unfavourable processing conditions, such as the presence of oxygen during the annealing of ohmic metal.

The pre-passivating properties of $\mathrm{Si}$ treatments and coatings are seen in the 2DEG sheet resistance values on the control sample, acid treated sample and $\mathrm{Si}$ treated/coated samples (Fig. 4.5). The values were taken for RTA anneals producing 


\begin{tabular}{|c|c|c|c|c|c|c|c|c|c|c|c|}
\hline & \multicolumn{2}{|c|}{$825^{\circ} \mathrm{C}$ RTA } & \multicolumn{3}{|c|}{$835^{\circ} \mathrm{C}$ RTA } & \multicolumn{3}{|c|}{$840^{\circ} \mathrm{C}$ RTA } & \multicolumn{3}{|c|}{$845^{\circ} \mathrm{C}$ RTA } \\
\hline & $R_{c}$ & $R_{s}$ & $R_{c}$ & $R_{s}$ & $I_{d}$ & $R_{c}$ & $R_{s}$ & $I_{d}$ & $R_{c}$ & $R_{s}$ & $I_{d}$ \\
\hline & Ohm-mm & $\mathrm{Ohm} / \mathrm{sq}$ & Ohm-mm & $\mathrm{Ohm} / \mathrm{sq}$ & $\mathrm{mA} / \mathrm{mm}$ & Ohm-mm & $\mathrm{Ohm} / \mathrm{sq}$ & $\mathrm{mA} / \mathrm{mm}$ & Ohm-mm & $\mathrm{Ohm} / \mathrm{sq}$ & $\mathrm{mA} / \mathrm{mm}$ \\
\hline${ }^{\dagger} \mathrm{B} 1$ & 091 & 398 & $237^{*}$ & $400^{*}$ & 500 & $106^{*}$ & $385^{*}$ & 637 & - & - & - \\
\hline $\mathrm{A} 1$ & 118 & 461 & $275^{*}$ & $425^{*}$ & 388 & $200^{*}$ & $425^{*}$ & 338 & - & - & - \\
\hline $\mathrm{S} 1$ & $227^{*}$ & $287^{*}$ & 068 & 305 & 1040 & 068 & 345 & 818 & - & - & - \\
\hline $\mathrm{S} 2$ & $625^{*}$ & $300^{*}$ & 074 & 293 & 1130 & 073 & 305 & 900 & - & - & - \\
\hline $\mathrm{L} 1$ & $550^{*}$ & $285^{*}$ & 069 & 325 & 1004 & 060 & 326 & 944 & - & - & - \\
\hline $\mathrm{H} 1$ & $308^{*}$ & $405^{*}$ & 058 & 300 & 1204 & 055 & 323 & 1067 & - & - & - \\
\hline $\mathrm{C} 1$ & $938^{*}$ & $638^{*}$ & 074 & 320 & 1149 & 045 & 330 & 1188 & 065 & 315 & 1146 \\
\hline $\mathrm{C} 2$ & - & - & - & - & - & 046 & 318 & 1020 & 060 & 318 & 1014 \\
\hline
\end{tabular}

Table 4.3: Batch-2 ohmic contact, 2DEG sheet resistances and current density over $2 \mu \mathrm{m}$ c-TLM gap from a four point probe measurement of c-TLM and TLM structures during RTA annealing. Averages of two to four measurements were taken for each sample. '*' denotes non-linearity in TLM data.

best ohmic contacts based on both ohmic contact resistance values and the saturation current densities. An exception was made for samples ${ }^{\dagger} \mathrm{B} 1$ and $\mathrm{A} 1$, where the lowest 2DEG sheet resistances were used. The fact that Si-treated and coated material showed signs of passivation after the ohmic metal RTA anneals but before the SiN passivation is very beneficial from the standpoint of in-process device performance monitoring, where process adjustments (such as additional ohmic contact RTA anneals) can still be made. 


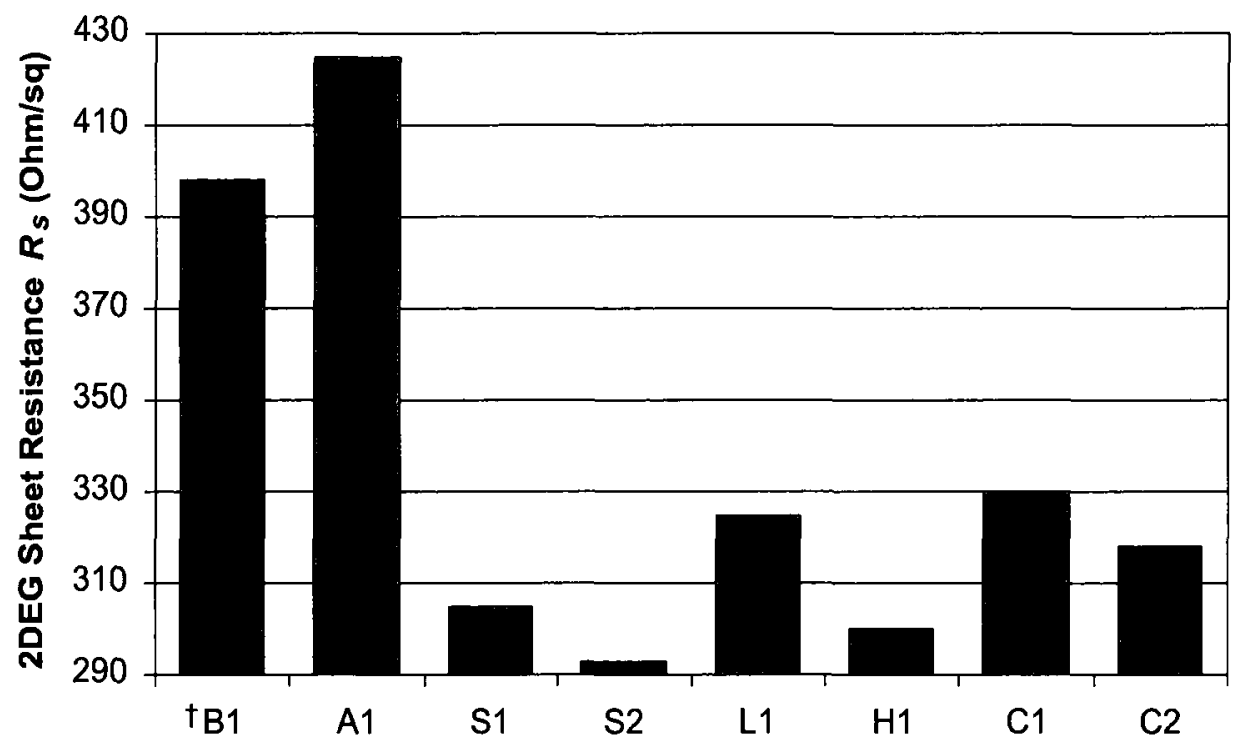

Figure 4.5: Batch-2 2DEG sheet resistance values $\left(\mathrm{R}_{s}\right)$ after ohmic contact RTA anneals. $835^{\circ} \mathrm{C}$ results were used for Si-treated samples. $840^{\circ} \mathrm{C}$ results were used for ${ }^{\dagger} \mathrm{B} 1, \mathrm{~A} 1$ and Si-coated samples. Pre-passivating effects of Si treatments and coatings can be clearly seen. 


\subsubsection{Batch-3}

Ohmic contact and 2DEG sheet resistances as well as the saturation current density over $2 \mu \mathrm{m}$ c-TLM gap at $9.9 \mathrm{~V}$ bias after RTA anneals at temperatures of 825 , 830 and $835^{\circ} \mathrm{C}$ are summarized in Table 4.4. The devices on Si-treated (S1, S2) and coated (C1, C2) samples have shown superior to the control samples saturation current densities at this stage, even in the absence of silicon nitride passivation films. The ohmic contact resistance values were comparable on all samples.

\begin{tabular}{|c|c|c|c|c|c|c|c|c|c|}
\hline & \multicolumn{3}{|c|}{$825^{\circ} \mathrm{C}$ RTA } & \multicolumn{3}{|c|}{$830^{\circ} \mathrm{C}$ RTA } & \multicolumn{3}{|c|}{$835^{\circ} \mathrm{C}$ RTA } \\
\hline & $R_{c}$ & $R_{s}$ & $I_{d}$ & $R_{c}$ & $R_{s}$ & $I_{d}$ & $R_{c}$ & $R_{s}$ & $I_{d}$ \\
\hline & Ohm-mm & $\mathrm{Ohm} / \mathrm{sq}$ & $\mathrm{mA} / \mathrm{mm}$ & Ohm-mm & $\mathrm{Ohm} / \mathrm{sq}$ & $\mathrm{mA} / \mathrm{mm}$ & Ohm-mm & $\mathrm{Ohm} / \mathrm{sq}$ & $\mathrm{mA} / \mathrm{mm}$ \\
\hline${ }^{\dagger} \mathrm{B} 1$ & 1.18 & 380 & 423 & 0.68 & 387 & 917 & - & - & - \\
\hline${ }^{\dagger} \mathrm{B} 2$ & 1.55 & 390 & 344 & 0.60 & 396 & 776 & - & - & - \\
\hline $\mathrm{S} 1$ & $4.00^{*}$ & $365^{*}$ & 490 & 0.53 & 352 & 1114 & - & - & - \\
\hline $\mathrm{S} 2$ & $3.00^{*}$ & $400^{*}$ & 535 & $0.45^{*}$ & $340^{*}$ & 809 & 0.68 & 292 & 1073 \\
\hline $\mathrm{C} 1$ & - & - & - & $2.00^{*}$ & $300^{*}$ & 645 & 0.59 & 266 & 1353 \\
\hline $\mathrm{C} 2$ & $3.65^{*}$ & $330^{*}$ & 532 & 0.58 & 290 & 1141 & - & - & - \\
\hline
\end{tabular}

Table 4.4: Batch-3 ohmic contact, 2DEG sheet resistances and current density over $2 \mu \mathrm{m}$ c-TLM gap from a four point probe measurement of c-TLM and TLM structures during RTA annealing. Averages of two to four measurements were taken for each sample. '*' denotes non-linearity in TLM data.

Figure 4.6 demonstrates again the pre-passivating effect from the Si treatment and coating on the 2DEG sheet resistances in AlGaN/GaN heterostructure in this batch. Since the contact resistance values were comparable across all samples and there were no accidental ohmic metal oxidation or over annealing, it was confirmed that this pre-passivation indeed occurred as a result of $\mathrm{Si}$ treatments and coatings. 


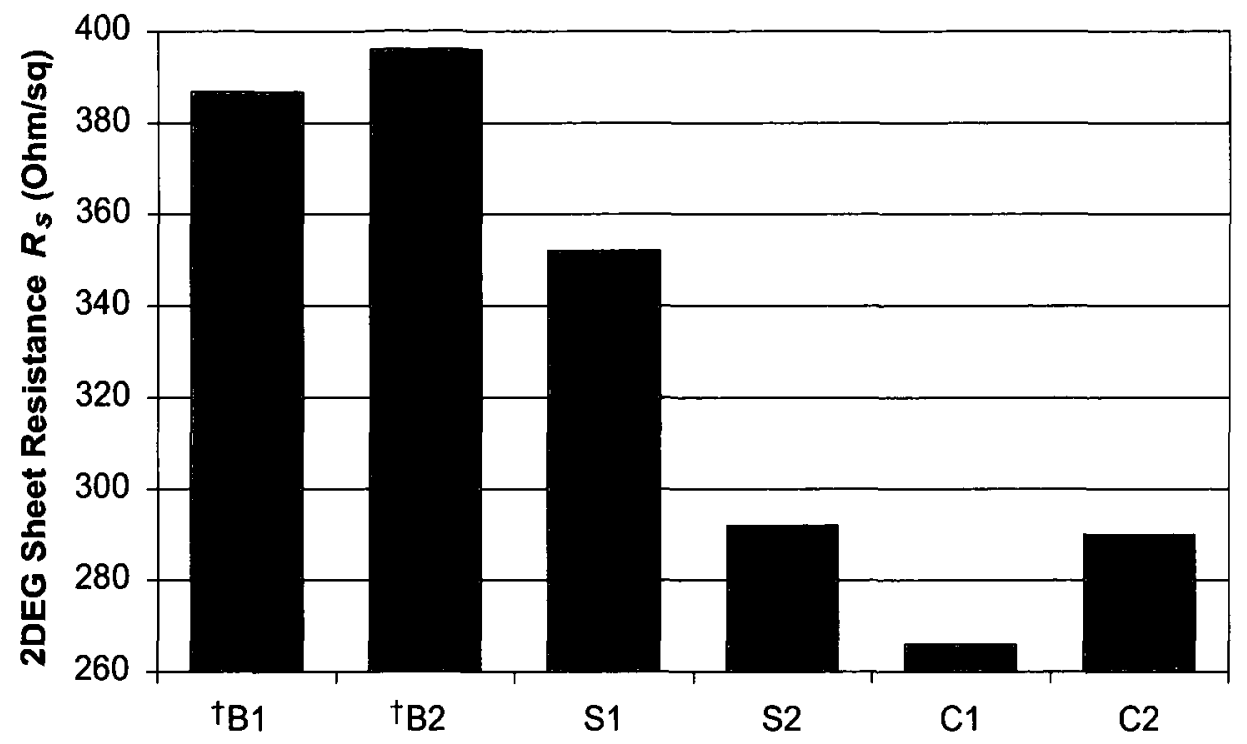

Figure 4.6: Batch-3 final 2DEG sheet resistance values $\left(\mathrm{R}_{s}\right)$ after ohmic contact RTA anneals. Pre-passivating effects of Si treatments and coatings can be clearly seen.

Table 4.5 summarizes Batch-3 TLM measurements after gate anneal and $1 \mathrm{ME}$ deposition processing steps. The Si-coated samples C1 and C2 demonstrated superior sheet resistances and current densities. These characteristics were further improved by the passivation. Samples S1 and S2 were likely affected by the processing steps performed for the water contact angle measurement during this fabrication run and thus did not exhibit high current densities and low sheet resistances before passivation with SiN. The Si treatment and coating did not produce any improvements to ohmic contacts. 


\begin{tabular}{|l|ccc|ccc|}
\hline & \multicolumn{3}{|c|}{ Gate Anneal } & \multicolumn{3}{c|}{ Passivation and 1ME Dep. } \\
\cline { 2 - 8 } & $R_{c}$ & $R_{s}$ & $I_{d}$ & $R_{c}$ & $R_{s}$ & $I_{d}$ \\
\cline { 2 - 8 } & Ohm-mm & Ohm/sq & $\mathrm{mA} / \mathrm{mm}$ & Ohm-mm & Ohm/sq & $\mathrm{mA} / \mathrm{mm}$ \\
\hline \hline${ }^{\dagger} \mathrm{B} 1$ c-TLM & - & - & 924 & 0.57 & 283 & 1053 \\
\hline${ }^{\dagger} \mathrm{B} 1$ TLM & 0.85 & 368 & - & 0.53 & 287 & - \\
\hline${ }^{\dagger} \mathrm{B} 2$ c-TLM & - & - & 837 & 0.61 & 295 & 945 \\
\hline${ }^{\dagger}$ B2 TLM & 1.02 & 386 & - & 0.53 & 287 & - \\
\hline S1 c-TLM & - & - & 784 & 0.55 & 288 & 1084 \\
\hline S1 TLM & 0.82 & 401 & - & 0.61 & 279 & - \\
\hline S2 c-TLM & - & - & 680 & 0.55 & 307 & 872 \\
\hline S2 TLM & 1.28 & 306 & - & 0.76 & 287 & - \\
\hline C1 c-TLM & - & - & 1037 & 0.56 & 278 & 1180 \\
\hline C1 TLM & 0.76 & 275 & - & 0.66 & 271 & - \\
\hline C2 c-TLM & - & - & 1050 & 0.54 & 276 & 1296 \\
\hline C2 TLM & 0.76 & 291 & - & 0.52 & 285 & - \\
\hline
\end{tabular}

Table 4.5: Batch-3 ohmic contact, 2DEG sheet resistances and current density over $2 \mu \mathrm{m}$ c-TLM gap from a four point probe measurement of c-TLM and TLM structures. Averages of two to six measurements were taken for each sample. $1 \mathrm{ME}$ TLM results combine the measurements of ohmic-probeable as well as of 100x64 and 100x32 $\mu \mathrm{m}$ 1ME-probeable TLMs.

The final ohmic contact and 2DEG sheet resistance values after the passivation and 1ME deposition are plotted in Fig. 4.7. It is evident from this figure that under the right processing conditions the control, Si-treated and Si-coated samples had comparable ohmic contact resistances. 


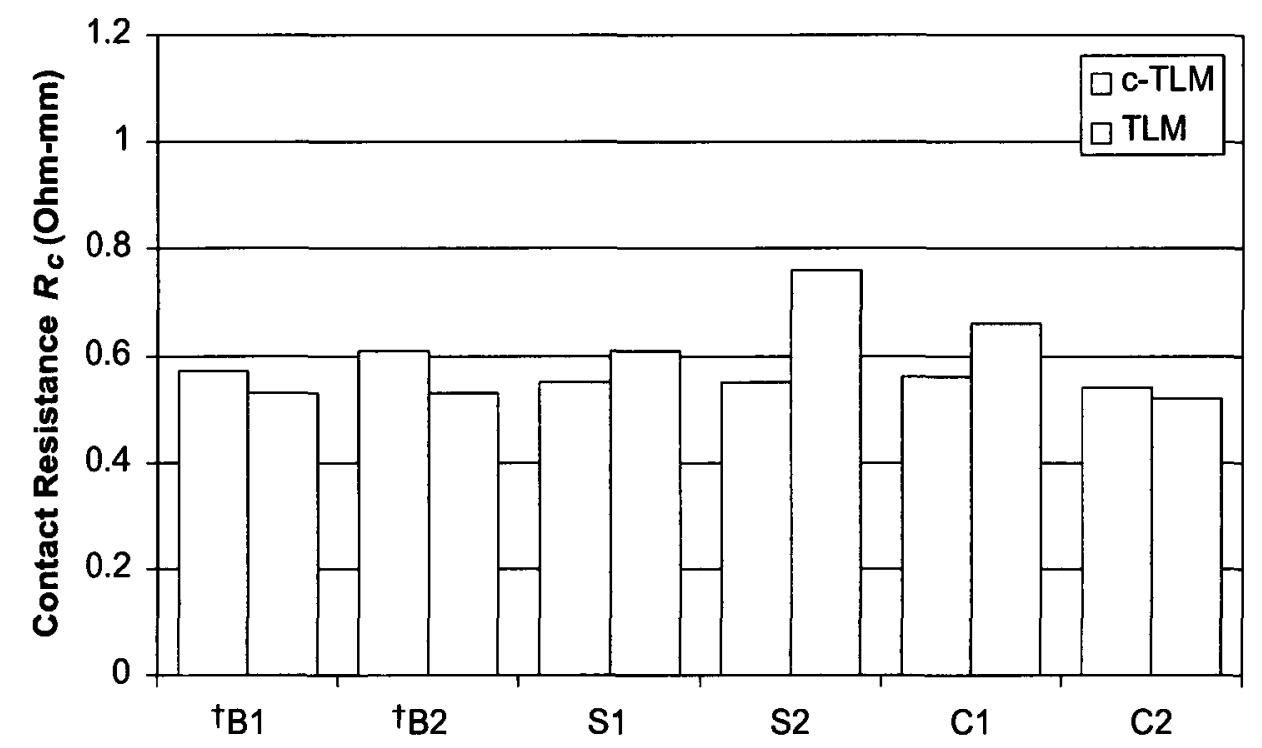

(a) Ohmic contact resistance $\left(\mathrm{R}_{c}\right)$ from c-TLM (red) and TLM (green)

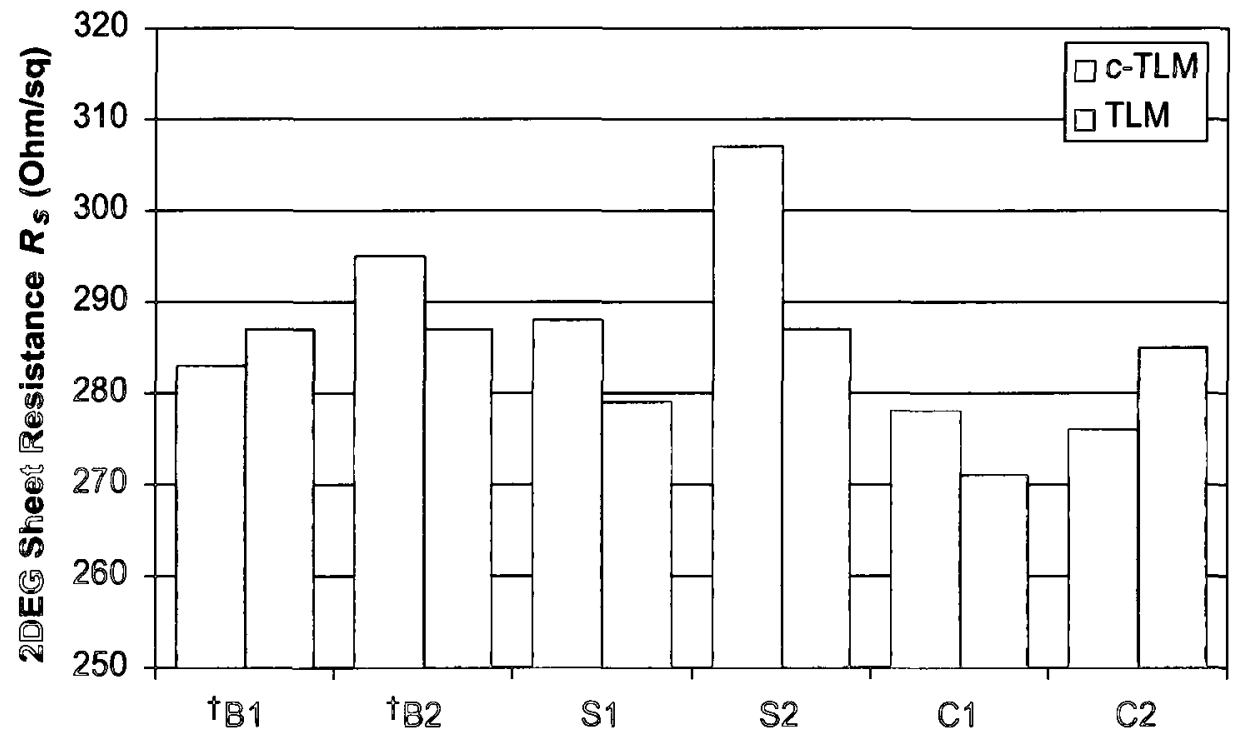

(b) 2DEG sheet resistance $\left(\mathrm{R}_{s}\right)$ from c-TLM (red) and TLM (green)

Figure 4.7: Batch-3 ohmic contact and 2DEG sheet resistances after passivation and $1 \mathrm{ME}$ deposition steps. 
Since the ohmic contacts on the control samples showed good resistance values after the anneals at $830^{\circ} \mathrm{C}$ and $835^{\circ} \mathrm{C}$ in Batch-3, it was concluded that the samples in Batch-1 were generally over annealed after $845^{\circ} \mathrm{C}$ RTA anneal. Because of heavy oxidation of the ohmic metal in Batch-2, only Batch-3 ohmic contact and 2DEG sheet resistance results were completely reliable. Thus the conclusions on the ohmic contact resistance values were taken from the results of this batch. The Si treatment and coatings were found to not affect the final values of the ohmic contact resistances significantly. The values remained in the range of 0.5 to $0.6 \mathrm{Ohm}-\mathrm{mm}$. It is worth pointing out, however, that NRC's processing of ohmic contacts was already highly optimized. Based on Batch-1 and Batch-2 results, Si treatments and coatings can positively influence the ohmic contact resistances in adverse (or poorly optimized) processing conditions. There is a clear evidence of beneficial pre-passivation effects due to Si treatment and coating exhibited in Batch-2 and Batch-3, while Batch-1 Si-treated samples displayed lower 2DEG sheet resistance than on control samples even after the passivation step. 


\subsection{Ohmic Contact Surface Morphology by Visual Inspection and Surface Profiling}

Batch-1 Si-treated samples S1 and S2 displayed a slightly better surface morphology and a similar line edge definition compared to the control and SiON-treated samples. The edge definition was worse on Si-coated samples in Batch-3. Batch-2 and Batch-3 Si-treated and Si-coated samples developed cracks in the ohmic metal stack following ohmic contact RTA anneals. The cracks in the ohmic metal were not noticed on Batch-1 samples. These defects created noticeable craters in $1 \mathrm{ME}$ overlayer upon further RTA annealing in Batch-3. The effect was most prominent on Si-coated samples (Fig. 4.8). It is clear that this phenomena is related to photoresist trapped in the cracks in the films which is not fully developed off, and thus results in a small explosion in the $1 \mathrm{ME}$ interconnect during annealing. Surface profiling with Dektak revealed a distinctive rise in the surface around the cracks $(300-500 \mathrm{~nm})$ and on the edges of the craters $(1.0-2.0 \mu \mathrm{m})$, quantifying the magnitude of the problem. This phenomenon is a serious practical problem for the use of this contact scheme in production. Due to the RTA failure during cooling, the ohmic metal on Batch-2 samples also displayed a significantly rougher morphology and the discolouration due to the oxidation. 


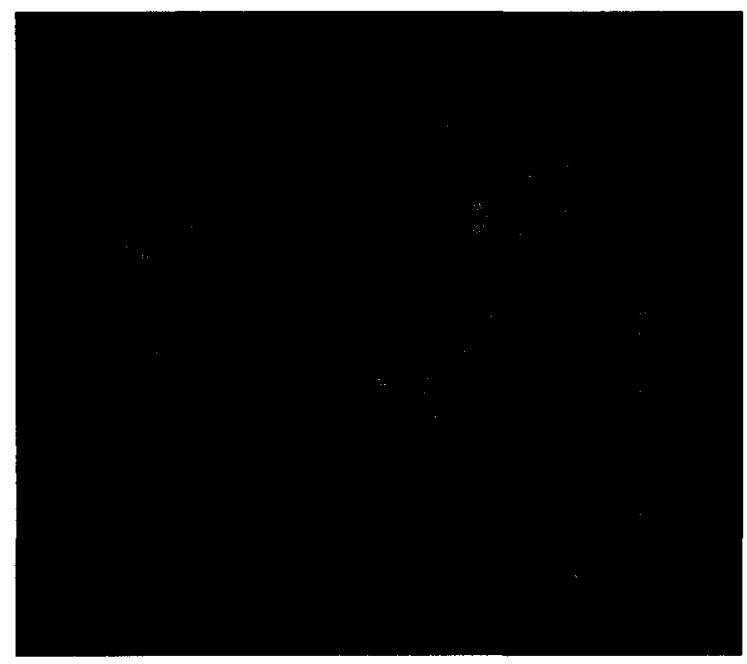

(a) Cracks in the ohmic metal stack after ohmic contact RTA anneals on sample C1 in Batch-2

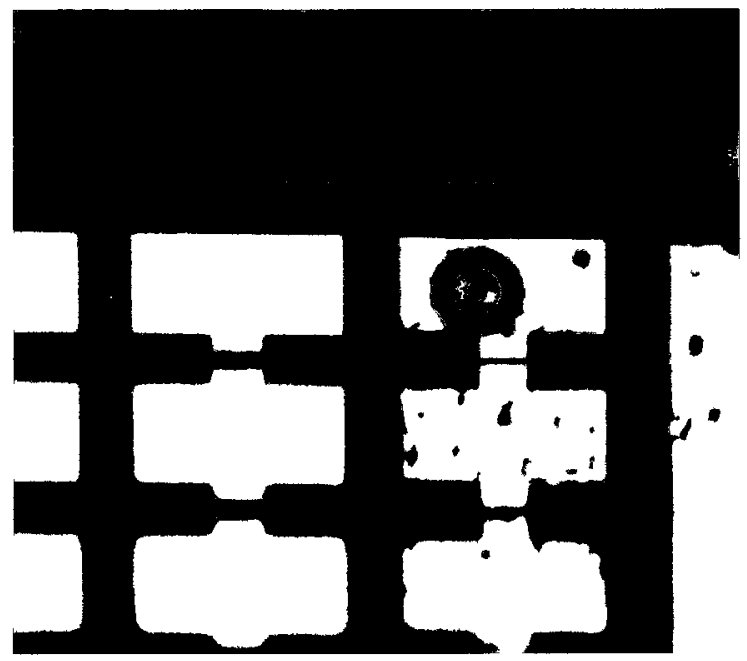

(b) A crater appearing in the places of the crack in the ohmic metal stack after 1ME anneal on sample $\mathrm{C} 1$ in Batch-3

Figure 4.8: Ohmic metal morphology problems and their effect on 1ME overlayer on samples $\mathrm{C} 1$ in Batch-2 and $\mathrm{C} 1$ in Batch-3. 


\subsection{HEMT DC and Pulsed I-V Curves/ Current \\ Collapse}

\subsubsection{Batch-1}

$\mathrm{DC}$ and pulsed I-V transistor curves are shown in Fig. 4.9, 4.10 and 4.11. Gate baseline voltage of $-5 \mathrm{~V}$, pulse period of $10 \mathrm{~ms}$ and pulse duration of $500 \mu$ s were used. A notable reduction in current collapse on unpassivated samples as well as improved DC current densities were clearly seen on Si-treated samples S1 and S2. Samples $\mathrm{O} 1$ and $\mathrm{O} 2$ did not demonstrate any improvements in these measurements.

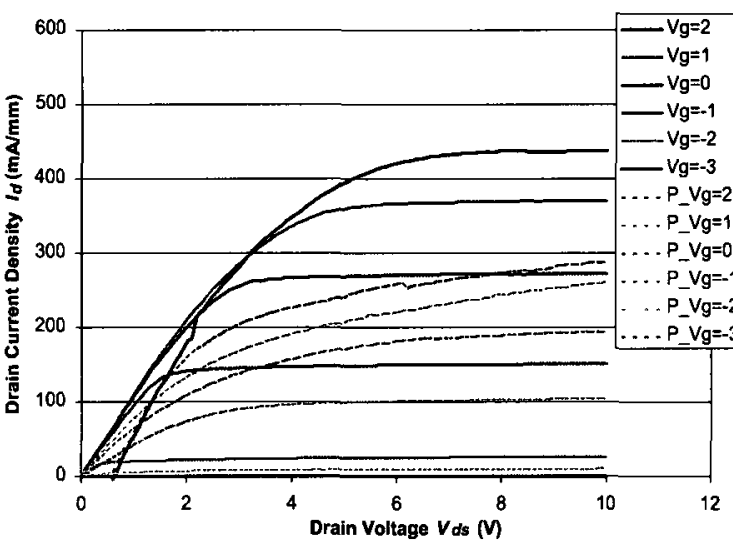

(a) ${ }^{\dagger}$ A1 unpassivated

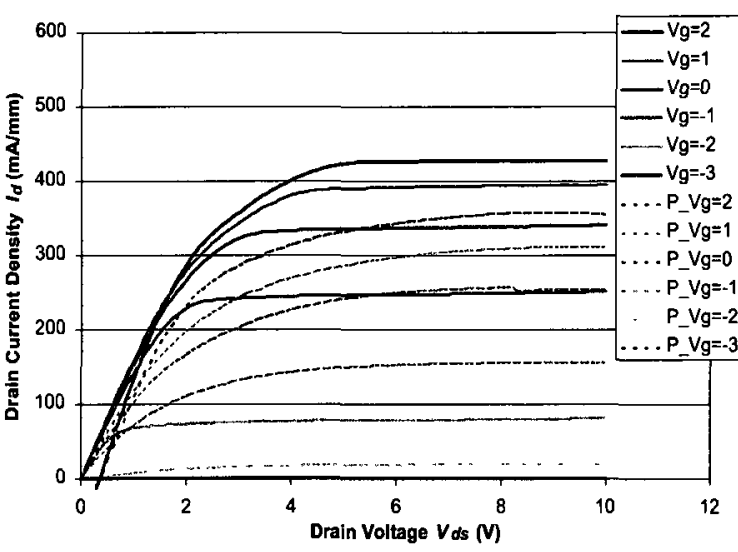

(c) B1 unpassivated

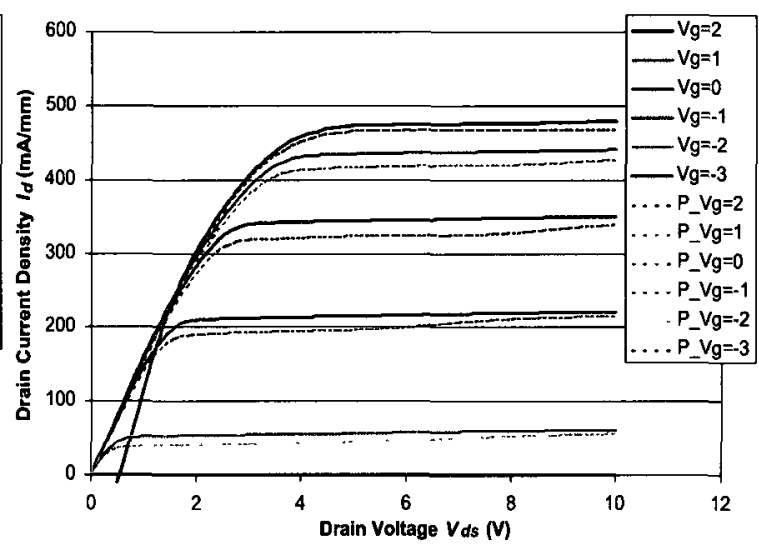

(b) ${ }^{\dagger} \mathrm{A} 1$ passivated $/ 1 \mathrm{ME}$

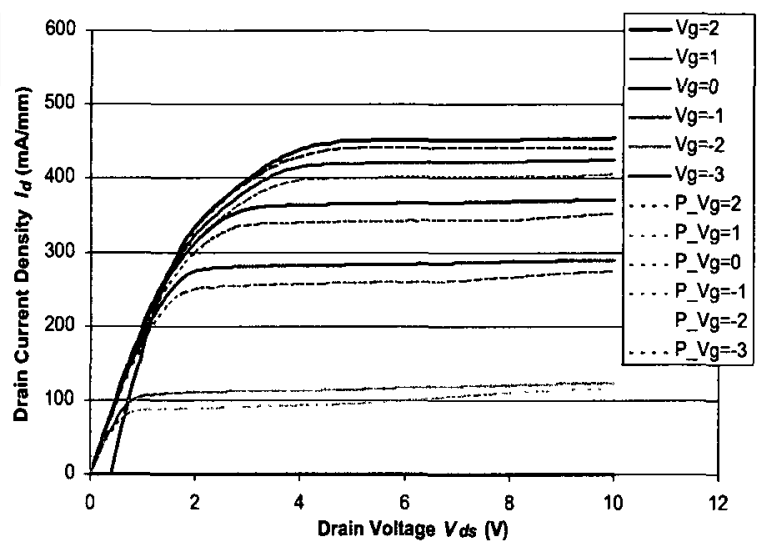

(d) B1 passivated/1ME

Figure 4.9: Batch-1 DC and pulsed I-V measurements of unpassivated and passivated HEMTs on samples ${ }^{\dagger} \mathrm{A} 1$ and $\mathrm{B} 1$. 


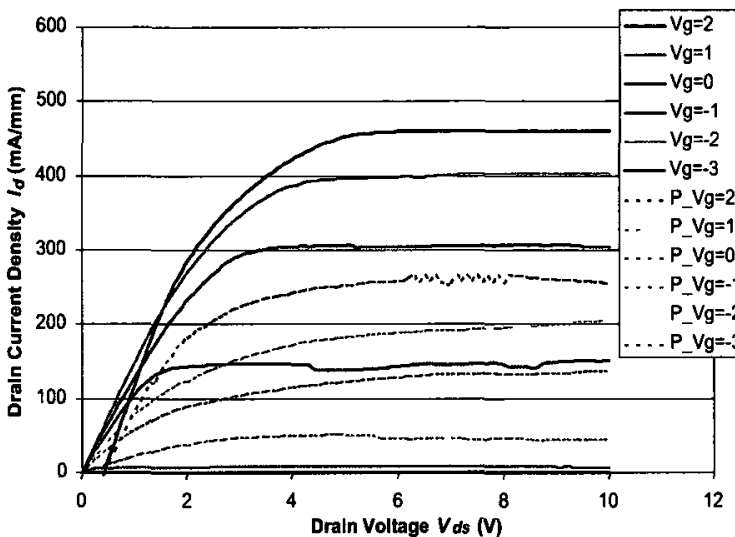

(a) B2 unpassivated

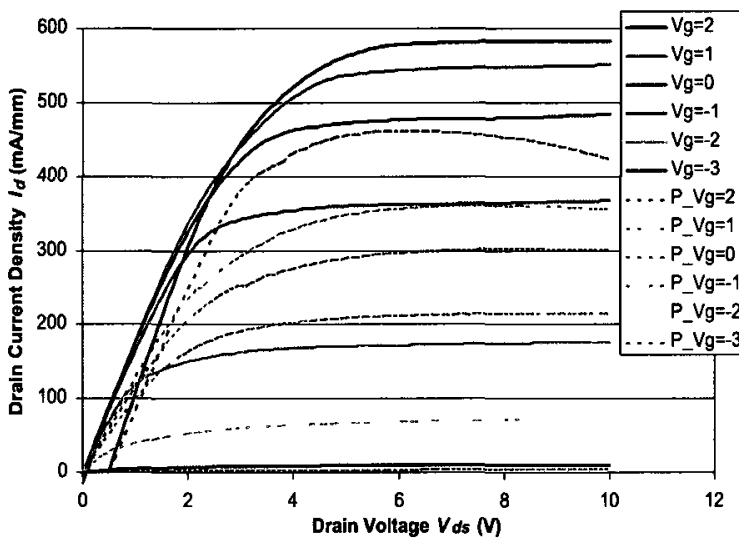

(c) S1 unpassivated

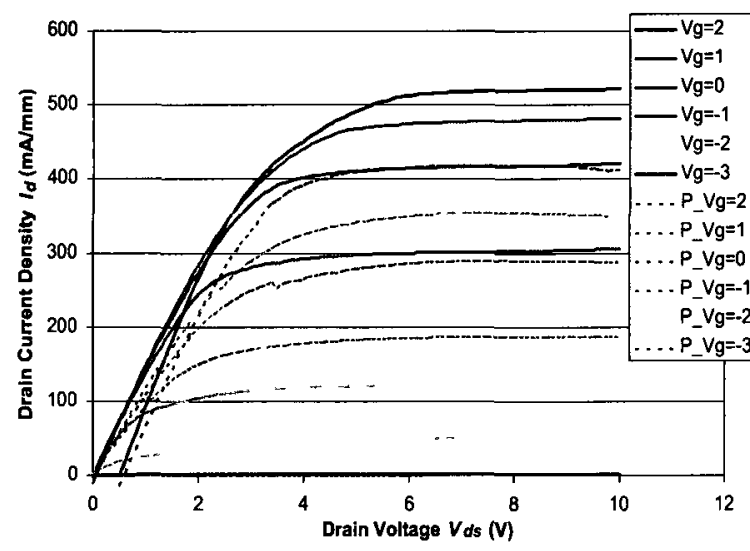

(e) S2 unpassivated

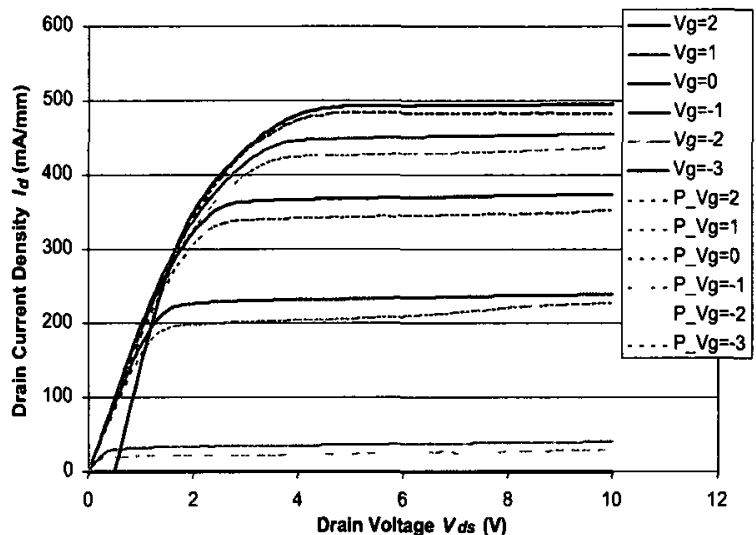

(b) B2 passivated/1ME

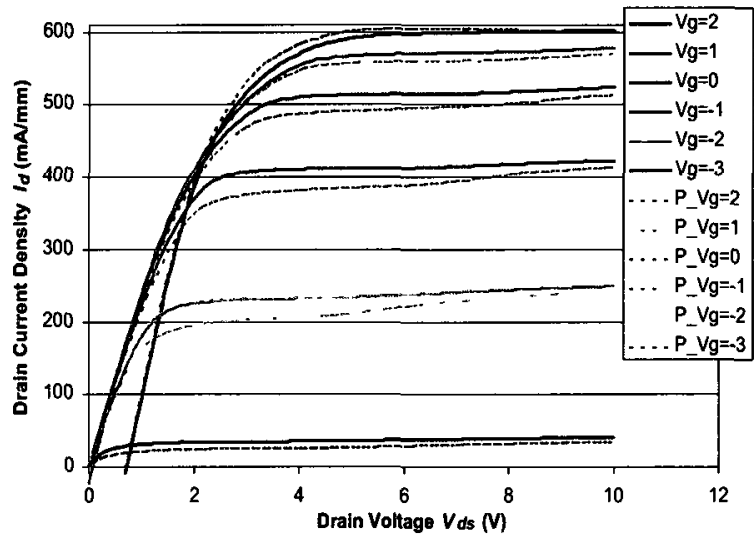

(d) S1 passivated/1ME

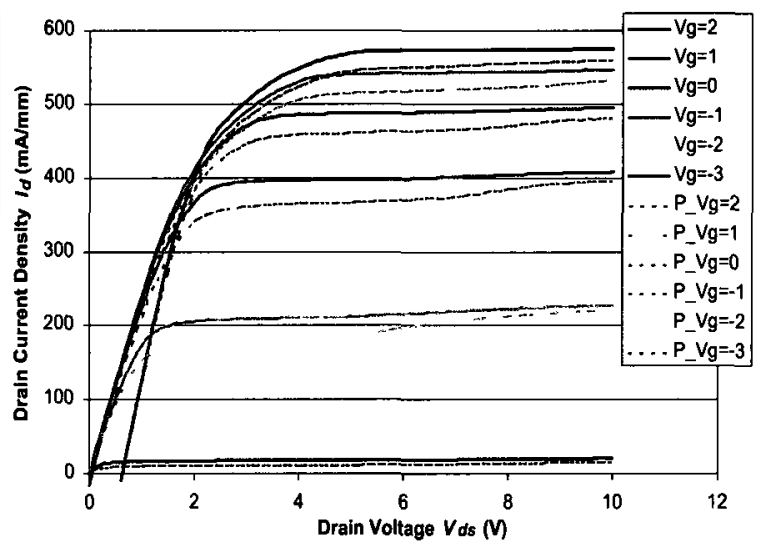

(f) S2 passivated/1ME

Figure 4.10: Batch-1 DC and pulsed I-V measurements of unpassivated and passivated HEMTs on samples B2, S1 and S2. 

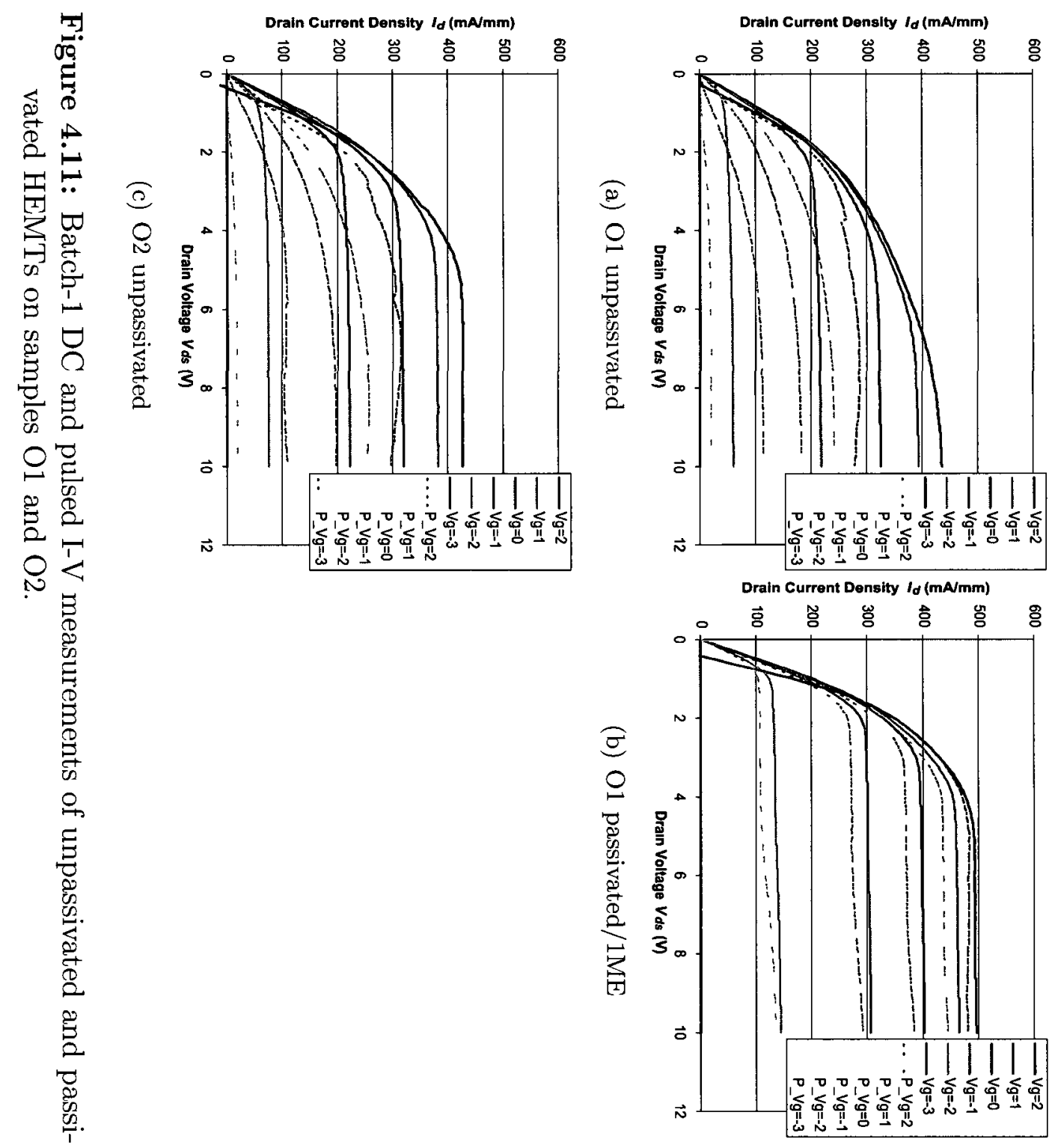


\subsubsection{Batch-3}

DC and pulsed I-V transistor curves are shown in Fig. 4.12, 4.13 and 4.14. Gate baseline voltage of $-8 \mathrm{~V}$ (Si-coated samples required lower voltage to fully pinch off), pulse period of $10 \mathrm{~ms}$ and pulse duration of $500 \mu \mathrm{s}$ were used. The Si-coated samples $\mathrm{C} 1$ and $\mathrm{C} 2$ displayed a significant reduction in current collapse even before the SiN passivation step. Following passivation, both current densities and current collapse improved even further on samples C1 and C2. Samples S1 and S2 exhibited worse current collapse than seen on control samples before the passivation, in contrast with the results from Batch-1. Additional processing steps performed for the water contact angle measurements were thought to be responsible for this result.

Following the passivation, transistor DC peak current densities for the gate voltage of $2 \mathrm{~V}$ reached the value of $915 \mathrm{~mA} / \mathrm{mm}$ on the control sample, $1,050 \mathrm{~mA} / \mathrm{mm}$ on Si-treated sample and $1,090 \mathrm{~mA} / \mathrm{mm}$ on Si-coated sample. These results may be indicative of small 2DEG improvements due to Si treatments and coatings on AlGaN/GaN material present even after the passivation. 

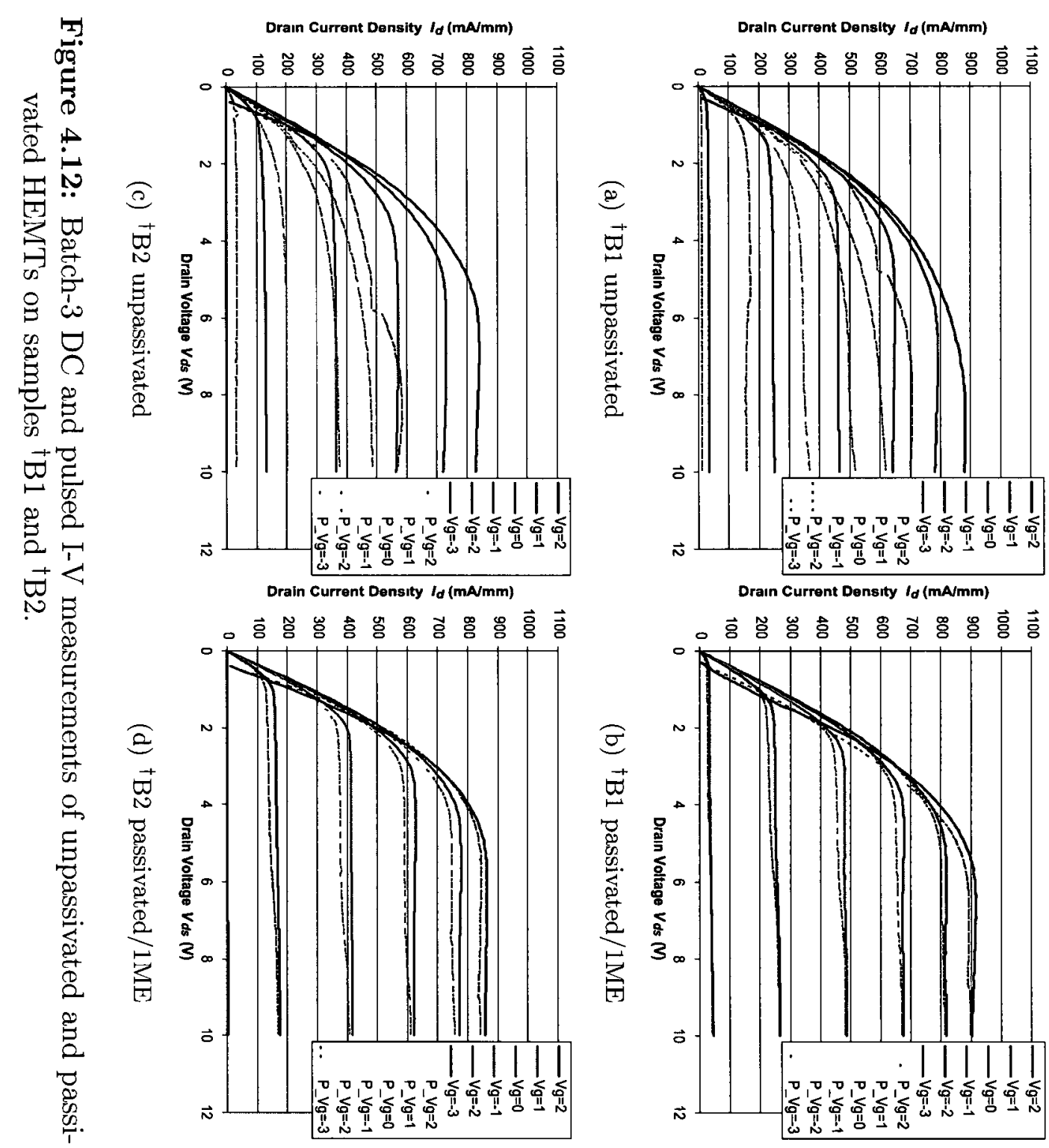

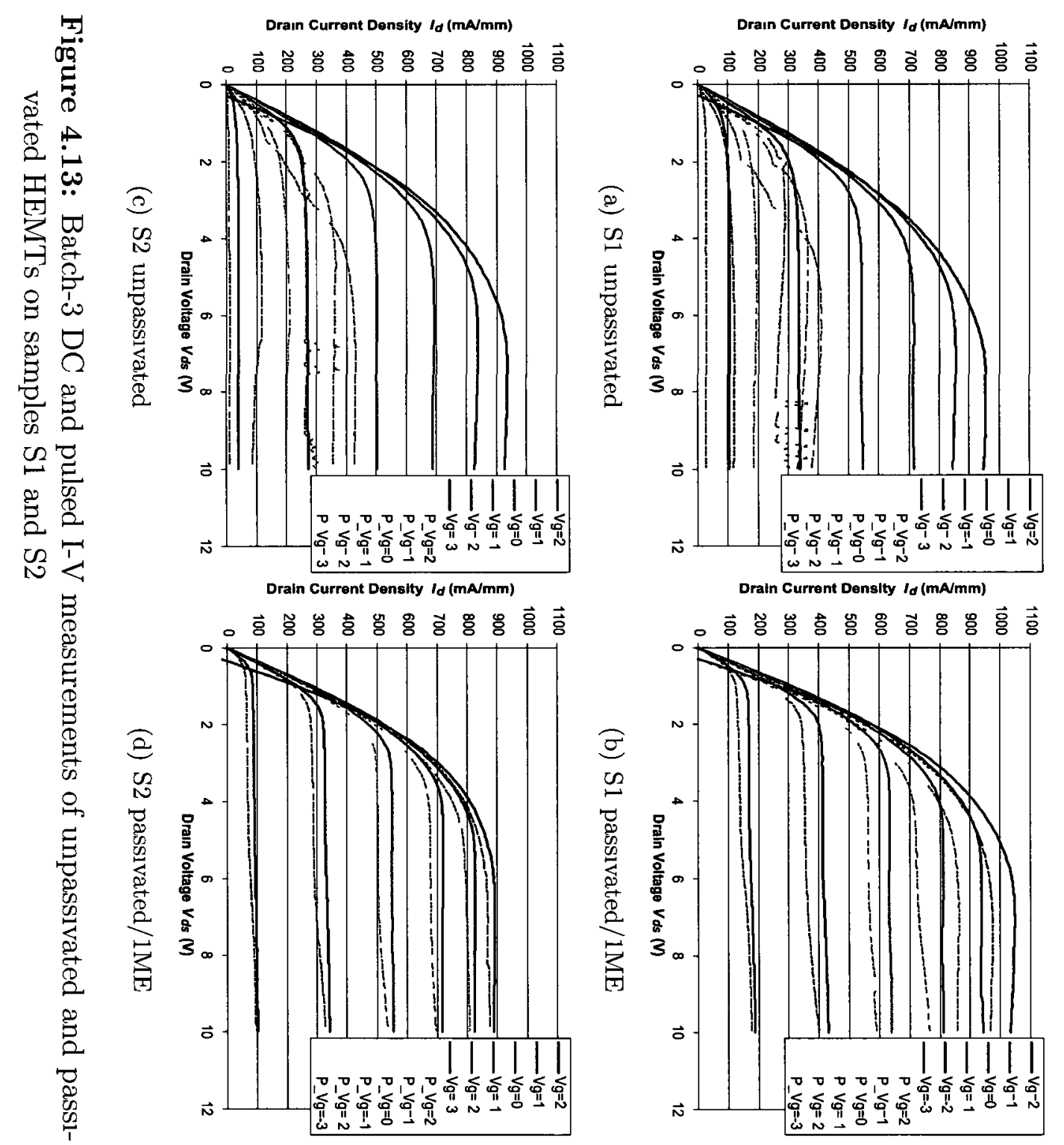

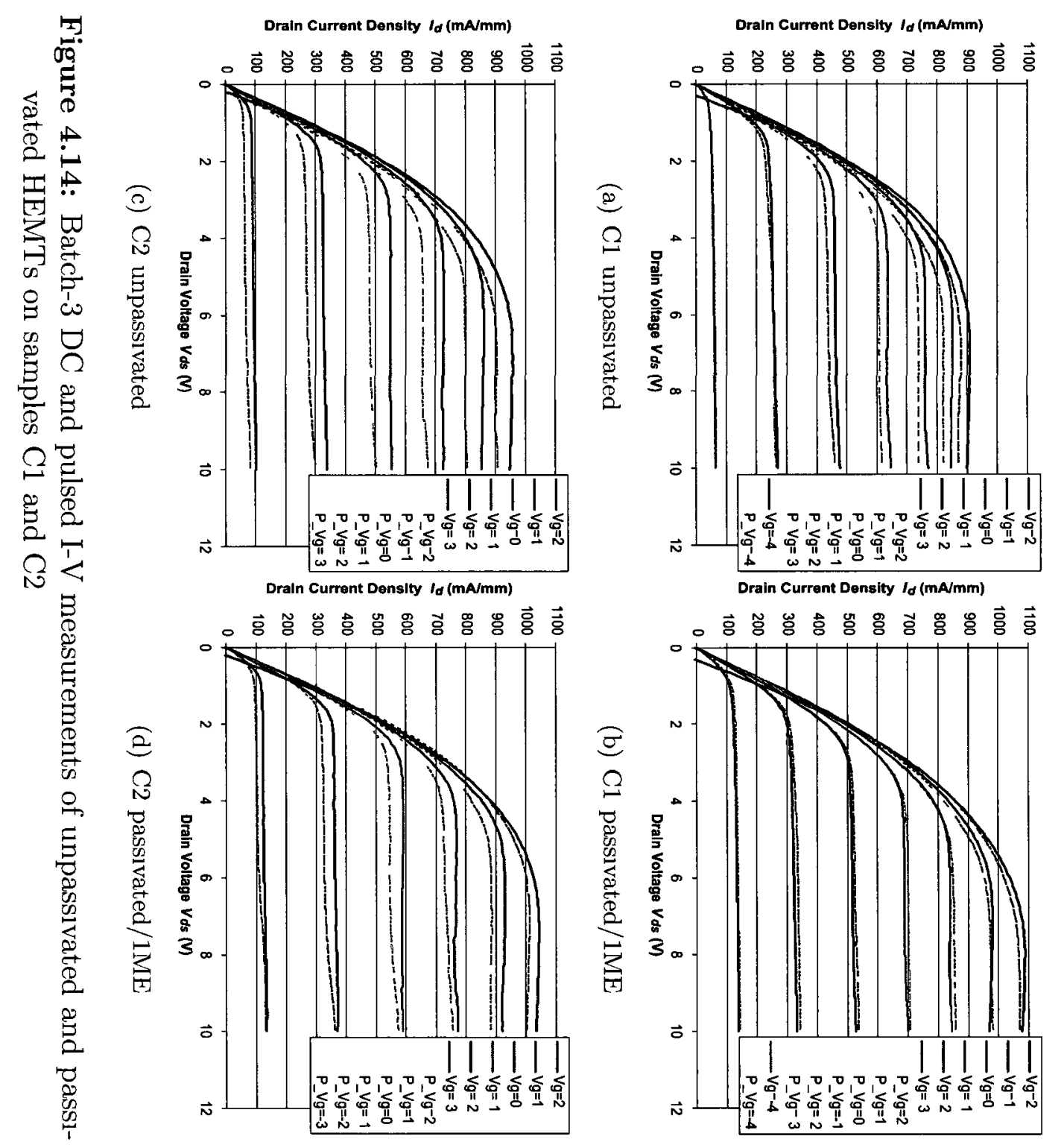


\subsection{HEMTs Threshold Voltage and Transconduc- tance}

\subsubsection{Batch-3}

Drain current as a function of gate and drain voltages were indicative of both the threshold voltage and the reverse leakage current in HEMTs on Batch-3 samples. The I-V curves are shown in Fig. 4.15 and 4.16. Note that the reverse leakage current is almost independent of drain bias for the Si-treated and Si-coated samples after passivation. Samples ${ }^{\dagger} \mathrm{B} 1$ and ${ }^{\dagger} \mathrm{B} 2$ as well as S1 and S2 were in agreement with each other. Leakage currents and threshold voltages were significantly different between samples $\mathrm{C} 1$ and $\mathrm{C} 2$, which is believed to be due to the additional processing steps performed for the water contact angle measurement on the sample C2. In general there is an increase in the magnitude of the threshold voltage on Si-treated and Sicoated samples. Unlike in the study performed by Onojima et al. [69], both the magnitude and changes in threshold voltages are significantly smaller in this study, indicating that there is a significant difference either in the GaN material or transistor structure used by Onojima. 

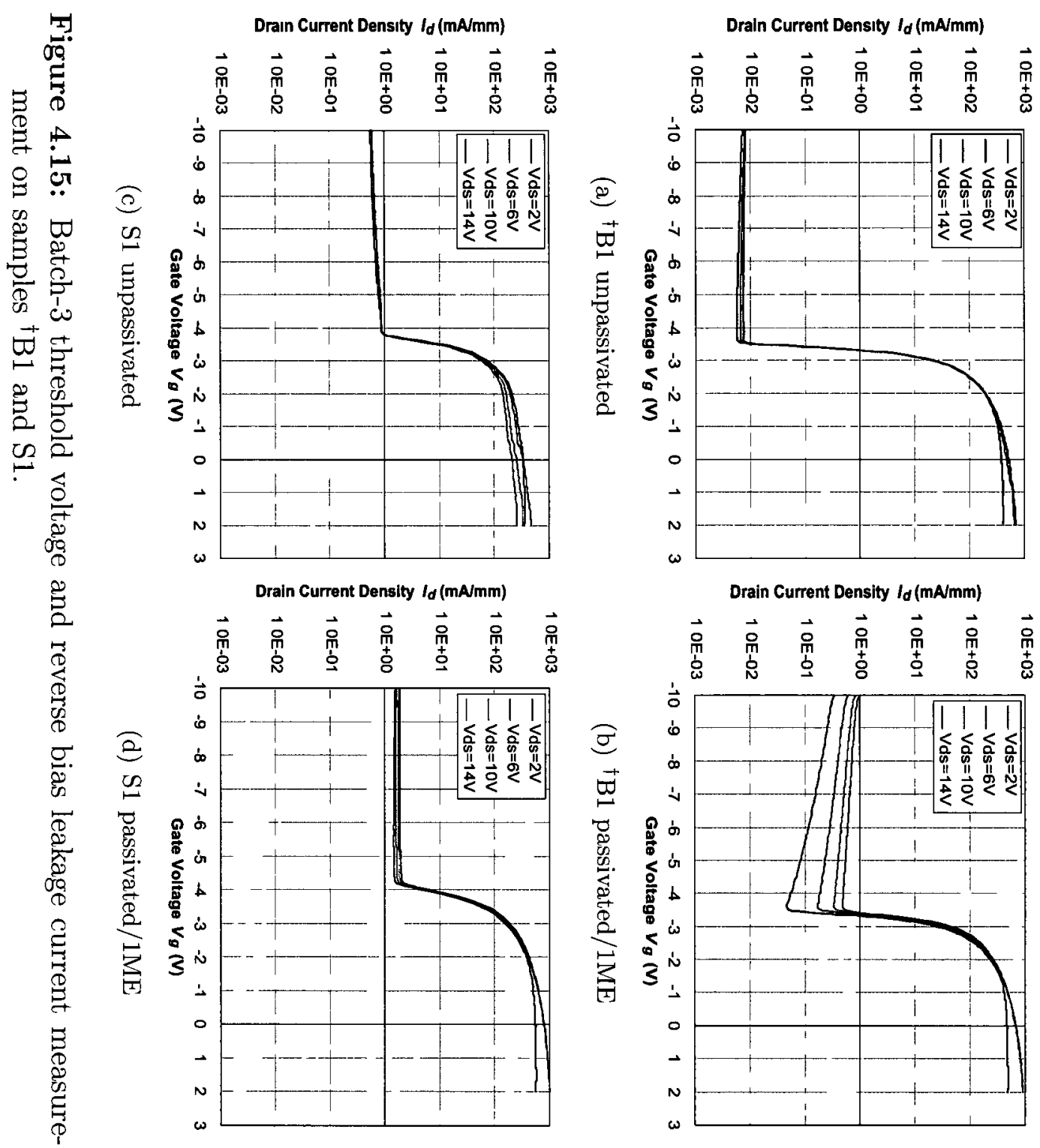

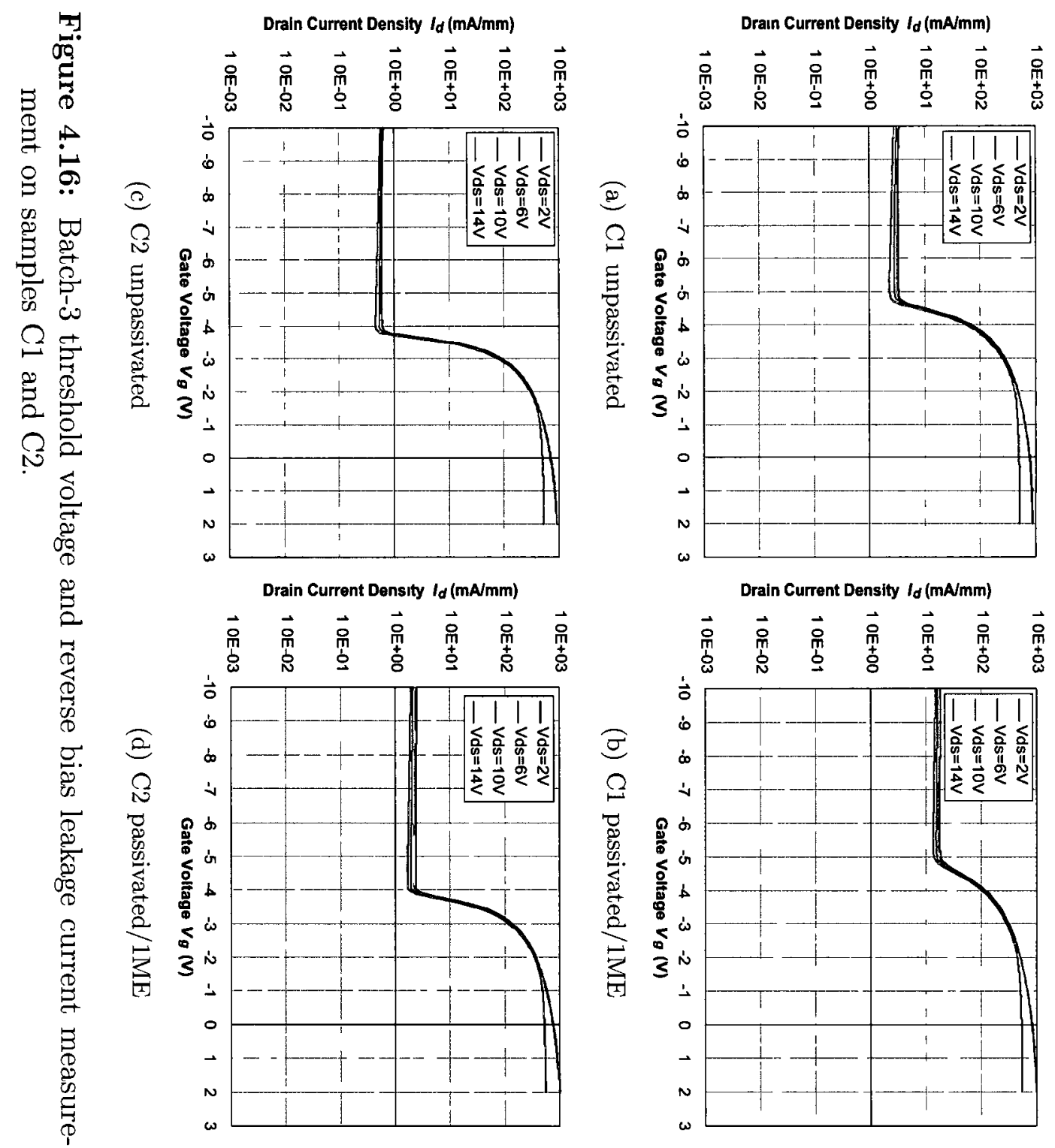
Typical plots resulting from HEMT transconductance measurements are shown in Fig. 4.17. Note that these plots were used to extract the actual values of the the threshold (pinch off) voltages of the devices.

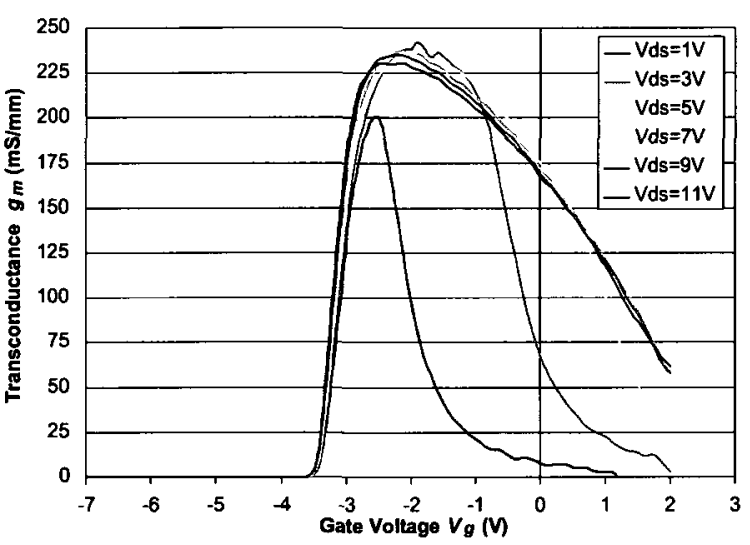

(a) ${ }^{\dagger} \mathrm{B} 1$ passivated/1ME

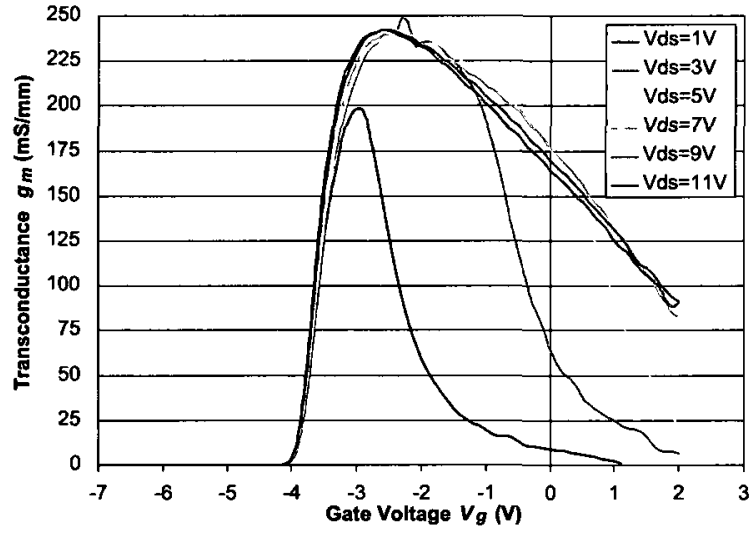

(b) $\mathrm{C} 2$ passivated/1ME

Figure 4.17: Batch-3 HEMTs transconductance measurements on samples ${ }^{\dagger} \mathrm{B} 1$ and $\mathrm{C} 2$.

Table 4.6 summarizes peak transconductances and threshold voltages for Batch3 HEMT devices. Additional processing steps performed on sample $\mathrm{C} 2$ for water contact angle measurement were thought to modify the thin Si film on the AlGaN surface, resulting in a different threshold voltage.

\begin{tabular}{|c|cc|cc|}
\hline & \multicolumn{2}{|c|}{ After Gate Anneal } & \multicolumn{2}{c|}{ Passivation/1ME } \\
\cline { 2 - 5 } & $V_{t h}(\mathrm{~V})$ & $g_{m(\max )}(\mathrm{mS} / \mathrm{mm})$ & $V_{t h}(\mathrm{~V})$ & $g_{m(\max )}(\mathrm{mS} / \mathrm{mm})$ \\
\hline \hline${ }^{\dagger} \mathrm{B} 1$ & -3.3 & 211 & -3.4 & 238 \\
\hline${ }^{\dagger} \mathrm{B} 2$ & -2.9 & 202 & -3.0 & 234 \\
\hline $\mathrm{S} 1$ & -3.8 & 205 & -4.1 & 244 \\
\hline $\mathrm{S} 2$ & -3.3 & 174 & -3.7 & 238 \\
\hline $\mathrm{C} 1$ & -4.7 & 212 & -4.9 & 213 \\
\hline $\mathrm{C} 2$ & -3.7 & 239 & -3.9 & 242 \\
\hline
\end{tabular}

Table 4.6: Batch-3 transconductance and threshold voltage summary. 


\subsection{HEMT Gate Schottky Junction I-V Curves}

\subsubsection{Batch-1}

Fig. 4.18 provides typical I-V curves of gate Schottky junction measurements on Batch-1 samples and Table 4.7 provides the summary of the results. Si-treated samples differed significantly in both ideality factors and saturation current densities under the reverse bias conditions compared to the controls, displaying better ideality factors and higher leakage currents. In addition, reverse currents were effectively flat after $-3 \mathrm{~V}$ was reached on the gate on samples $\mathrm{S} 1$ and $\mathrm{S} 2$. This result was consistent

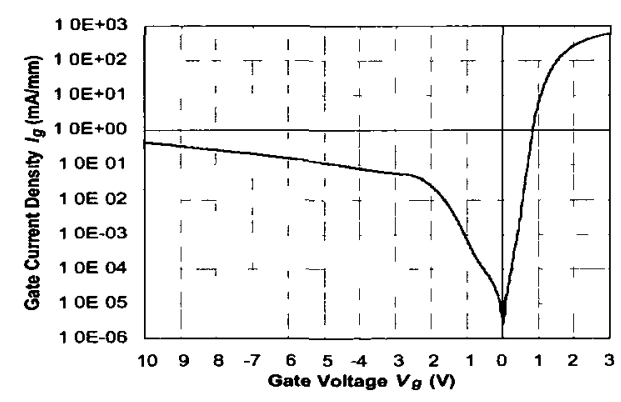

(a) ${ }^{\dagger} \mathrm{A} 1$, control

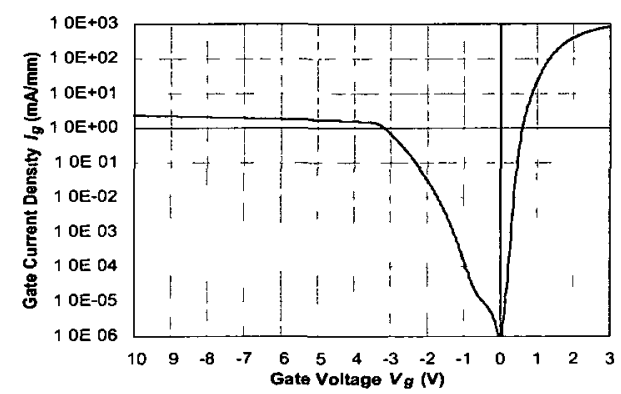

(c) S1, Si-treated

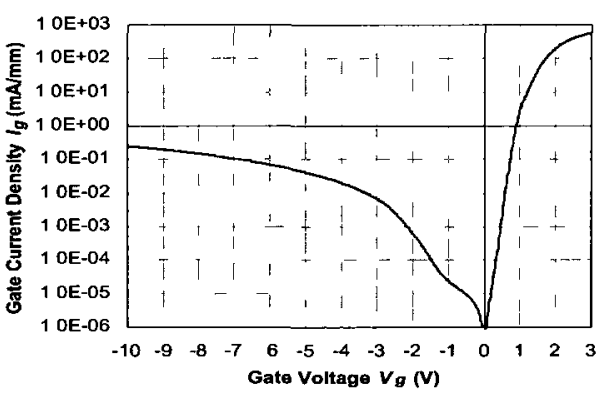

(b) B1, RTA annealed control

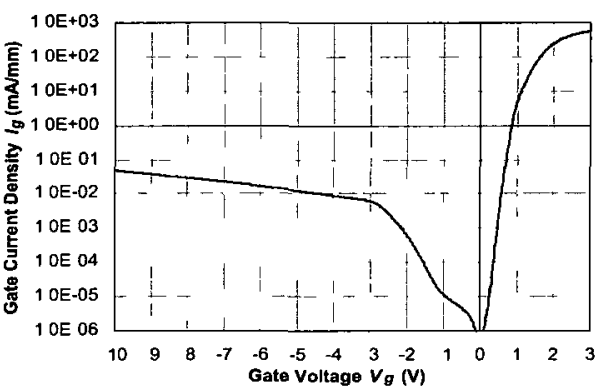

(d) O1, SiON-treated

Figure 4.18: Batch-1 gate Schottky junction I-V curves after the passivation and $1 \mathrm{ME}$ deposition steps.

with the reduction in Schottky barrier height due to the formation of $\mathrm{TiSi}_{x}$ compounds which in turn increased the thermionic emission current transport component. On the other hand, the anneal of the untreated samples B1 and B2 reduced the leakage 
current by a factor of two. The SiON treatment reduced the leakage current by one order of magnitude. Growth of thin oxides on the AlGaN surface is thought to be responsible for this effect.

\begin{tabular}{|c|c|c|c|}
\hline & \multicolumn{3}{|c|}{ After Passivation/1ME } \\
\hline & $I_{R,-10 \mathrm{~V}}(\mathrm{~mA} / \mathrm{mm})$ & $I_{F,+3 V}(\mathrm{~mA} / \mathrm{mm})$ & $n$ \\
\hline \hline${ }^{\dagger} \mathrm{A} 1$ & 0.45 & 630 & 2.30 \\
\hline $\mathrm{B} 1$ & 0.25 & 550 & 2.30 \\
\hline $\mathrm{B} 2$ & 0.24 & 630 & 2.30 \\
\hline $\mathrm{S} 1$ & 2.4 & 850 & 1.26 \\
\hline $\mathrm{S} 2$ & 2.5 & 775 & 1.22 \\
\hline $\mathrm{O} 1$ & 0.048 & 580 & 1.76 \\
\hline
\end{tabular}

Table 4.7: Batch-1 gate Schottky junction I-V summary after passivation and 1ME dep. processing steps.

\subsubsection{Batch-3}

Table 4.8 summarizes Batch-3 Schottky junction measurement results. A noticeable difference existed between samples $\mathrm{C} 1$ and $\mathrm{C} 2$ in leakage currents and ideality factors and is believed to be the result of the additional processing steps performed for the water contact angle measurement on the sample $\mathrm{C} 2$.

\begin{tabular}{|c|c|c|c|c|}
\hline & \multicolumn{4}{|c|}{ After Passivation/1ME } \\
\hline & $I_{R,-40 \mathrm{~V}}(\mathrm{~mA} / \mathrm{mm})$ & $I_{R,-10 \mathrm{~V}}(\mathrm{~mA} / \mathrm{mm})$ & $I_{F,+3 V}(\mathrm{~mA} / \mathrm{mm})$ & $n$ \\
\hline \hline${ }^{\dagger} \mathrm{B} 1$ & 3.8 & 0.44 & 790 & 1.95 \\
\hline${ }^{\dagger} \mathrm{B} 2$ & 3.0 & 0.85 & 970 & 1.95 \\
\hline $\mathrm{S} 1$ & 4.0 & 2.7 & 800 & 1.44 \\
\hline $\mathrm{S} 2$ & 8.6 & 3.8 & 840 & 1.50 \\
\hline $\mathrm{C} 1$ & 46 & 23 & 800 & 1.44 \\
\hline $\mathrm{C} 2$ & 6.7 & 3.8 & 610 & 2.05 \\
\hline
\end{tabular}

Table 4.8: Batch-3 gate Schottky junction I-V summary after passivation and 1ME dep. processing steps. 


\subsection{Drain-to-Source Breakdown Voltage}

\subsubsection{Batch-1}

The devices on the control and $\mathrm{Si}$ - and SiON-treated samples were not found to break down at drain to source voltages of up to $150 \mathrm{~V}$ (measurement power limits were reach on some devices before $200 \mathrm{~V}$ bias was reached). However, Si-treated samples exhibited higher leakage current than control samples, while SiON-treated samples showed lower leakage than controls for a wide range of the bias conditions. Fig. 4.19 shows typical I-V curves obtained from these measurements.

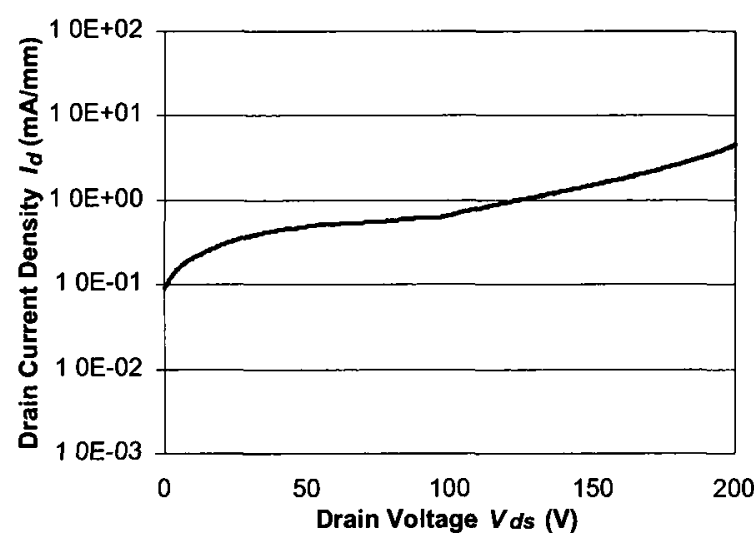

(a) ${ }^{\dagger} \mathrm{A} 1$, control

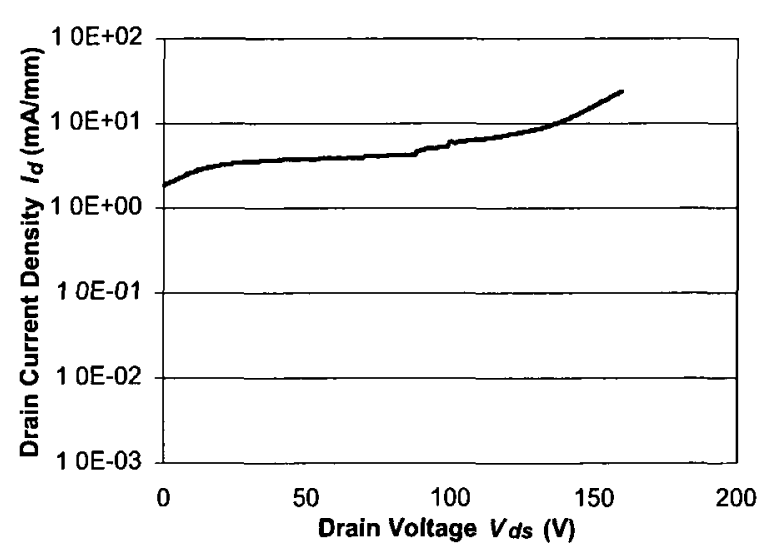

(c) S2, Si-treated

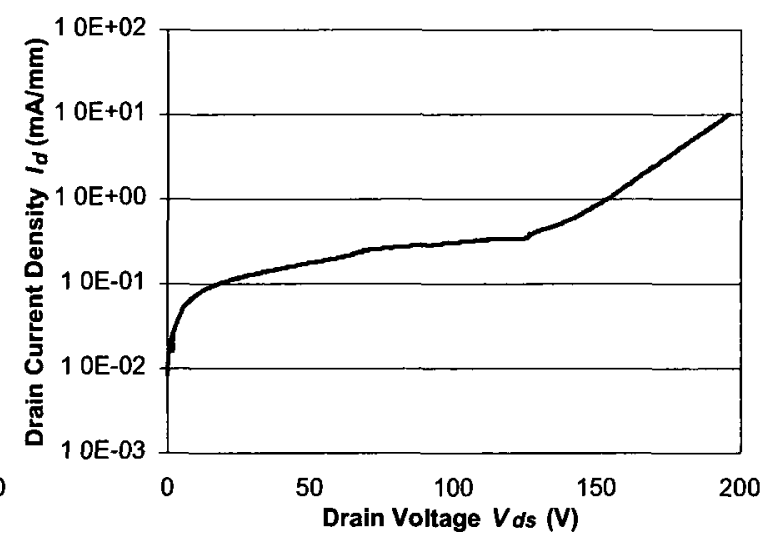

(b) B1, RTA annealed control

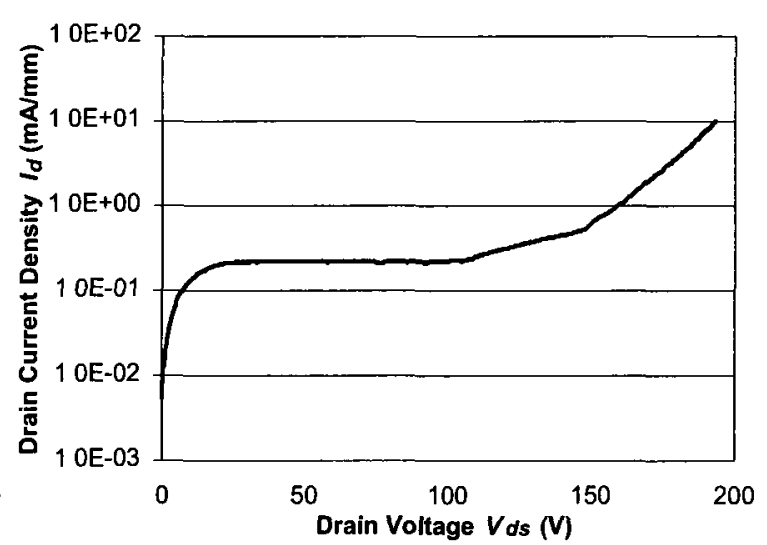

(d) O1, SiON-treated

Figure 4.19: Batch-1 drain-to-source breakdown voltage measurements. Gate voltage $\left(V_{g}\right)$ was set to $-5 \mathrm{~V}$. 


\subsection{SIMS AlGaN/GaN Depth Profiling}

The SIMS profile of ${ }^{27} \mathrm{Al}$ on the sample ${ }^{\dagger} \mathrm{B} 1$ from Batch- 2 was used to establish a rough estimate of the profiling depth as a function of time. The peak in Al signal at the time of 40 seconds corresponded to the AlN spacer under $20 \mathrm{~nm}$ of AlGaN barrier layer, giving ion etch rate of $0.5 \mathrm{~nm} / \mathrm{sec}$ (Fig. 4.20). Fig. 4.21 provides $\mathrm{Si}$ SIMS profiles for the reference sample R1, control sample ${ }^{\dagger} \mathrm{B} 1$ and Si-treated samples S1, L1 and $\mathrm{H} 1$ after the ohmic contact deposition and ohmic annealing processing steps. The difference in profiles between control sample ${ }^{\dagger} \mathrm{B} 1$ (which had no Si surface treatments) and the other samples is striking. It is clear that even on samples S1, L1 and $\mathrm{H} 1$, which were subjected to an aggressive $\mathrm{Si}$ wet etch, very large amounts of $\mathrm{Si}$ remain on the surface region. For all these samples the initial Si signal is comparable to that of reference R1, which had pure Si left in place on the surface.

The thickness of the remnant Si rich film scaled according to RTA temperatures. Higher temperatures produced thicker remnant films as can be seen on the first $5 \mathrm{~nm}$ of the Si SIMS profile. Note that the profiling depth inferred from the SIMS profile of $\mathrm{Al}$ was only a rough approximation in Si profiles due to different etching rates of AlGaN and Si films. Although the target thickness for the Si film on the reference sample was $5 \mathrm{~nm}$, surface profiling with Dektak resulted in a value of $7.3 \mathrm{~nm}$. Thicker than $5 \mathrm{~nm}$ film thickness values were also obtained on Batch-3 samples, likely resulting from the growth of native oxide on the Si surface. By comparing the profiles of the Sitreated samples to the reference sample, $\mathrm{S} 1$ and $\mathrm{H} 1$ were estimated to contain about $2.4 \mathrm{~nm}$ of the Si-containing film.

All Si-treated samples showed a long "tail" of Si signal apparently extending to a depth of almost $20 \mathrm{~nm}$. This is almost certainly an artifact resulting from forward scattering of $\mathrm{Si}$ from the surface layer, driven by the Cs ion analysis beam. Note that samples L1 and H1 show very similar Si profiles, despite the large difference in 
anneal temperatures.

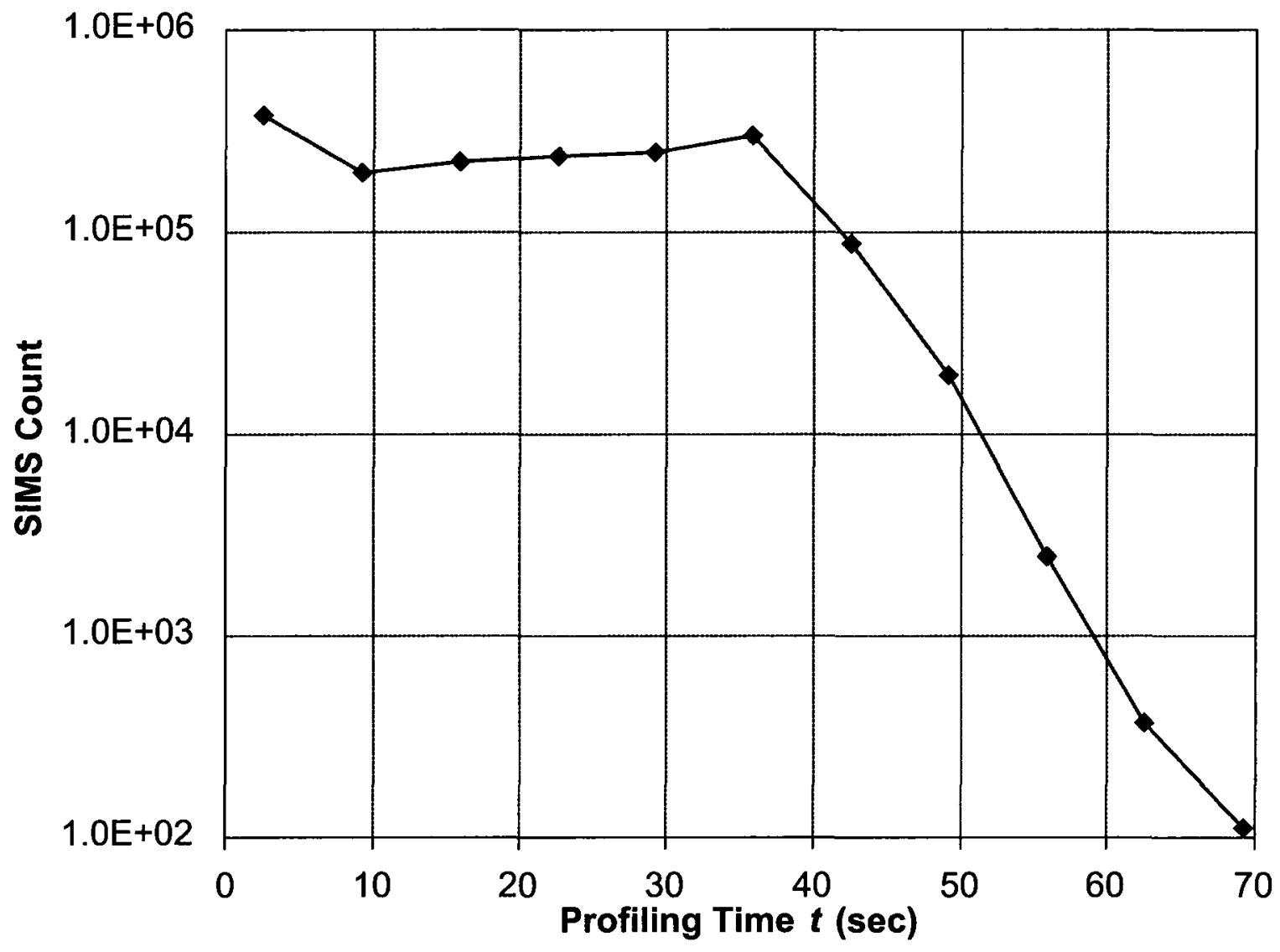

Figure 4.20: Batch-2 SIMS ${ }^{27} \mathrm{Al}$ profile of the control sample ${ }^{\dagger} \mathrm{B} 1$. 


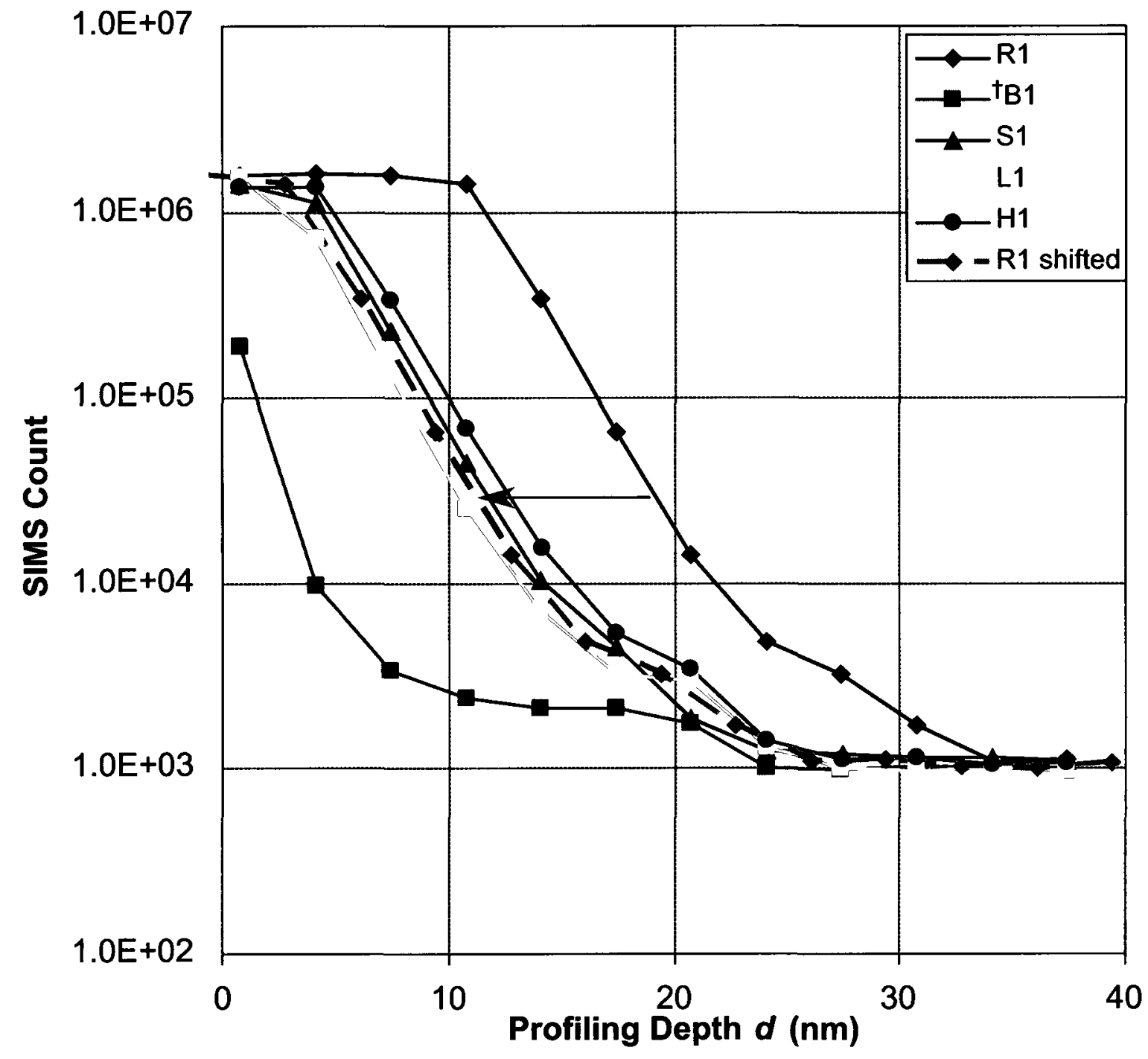

Figure 4.21: Batch-2 SIMS ${ }^{28} \mathrm{Si}$ profile of the control sample as well as Si-treated and Si-coated reference samples. 


\subsection{AlGaN Surface Chemical Composition and Film Thickness by XPS and AR-XPS}

Shirley background subtraction was performed on all XPS spectra. The elemental Si $2 p$ peak at $99.3 \mathrm{eV}$ was used for charge referencing. The spin-orbit splitting ratio of 1:2 with the peaks separated by $0.6 \mathrm{eV}$ was used for fitting of the Si $2 p$ peaks. On samples which did not contain elemental $\mathrm{Si}, \mathrm{Ga} 3 p$ peaks with the area ratios of 1:2 due to the spin-orbit splitting of the $p$ orbital in the binding energy window of $105 \mathrm{eV}$ to $110 \mathrm{eV}$ were used for spectra alignment. The position of the $\mathrm{Ga} 3 p$ peaks with respect to the $\mathrm{Si} 2 p$ peak was established from the Ar sputtered reference sample R2, Batch-2.

Additional peaks were used where necessary to fit the XPS data in order to account for the $\mathrm{Si}^{1+}, \mathrm{Si}^{2+}$ and $\mathrm{Si}^{3+}$ components [76], where the $\mathrm{Si}$ in elemental state and in $\mathrm{SiO}_{2}$ compound possessed $\mathrm{Si}^{0}$ and $\mathrm{Si}^{4+}$ oxidation states respectively.

The Gaussian-Lorentzian peak shape fitting was initially used, however, the best fits were achieved when $100 \%$ Gaussian peak shape fitting was performed.

Fig. 4.22 provides the XPS spectra and peak fitting results for sample R2 at three stages of Ar sputtering. By setting the peak location to $99.3 \mathrm{eV}$ for elemental $\mathrm{Si}, \mathrm{Ga}$ $3 p$ peaks were found to be located at 108.9 and $105.6 \mathrm{eV}$. Note that the two Si $2 p$ peaks for $\mathrm{SiO}_{x}$ compounds were not sufficient to accurately represent all oxidation states of $\mathrm{Si}$ present in Ar sputtered film. However, the analysis relied on the elemental Si peak, the location of which was not significantly affected by the fitting of $\mathrm{SiO}_{x}$ peaks. The elemental Si peak had a constant Full Width at Half Maximum (FWHM). 


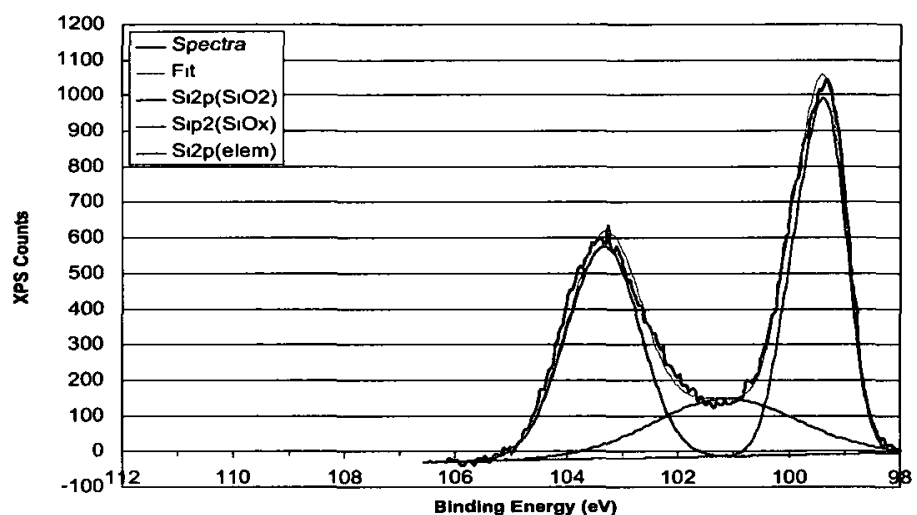

(a) Before Ar sputtering

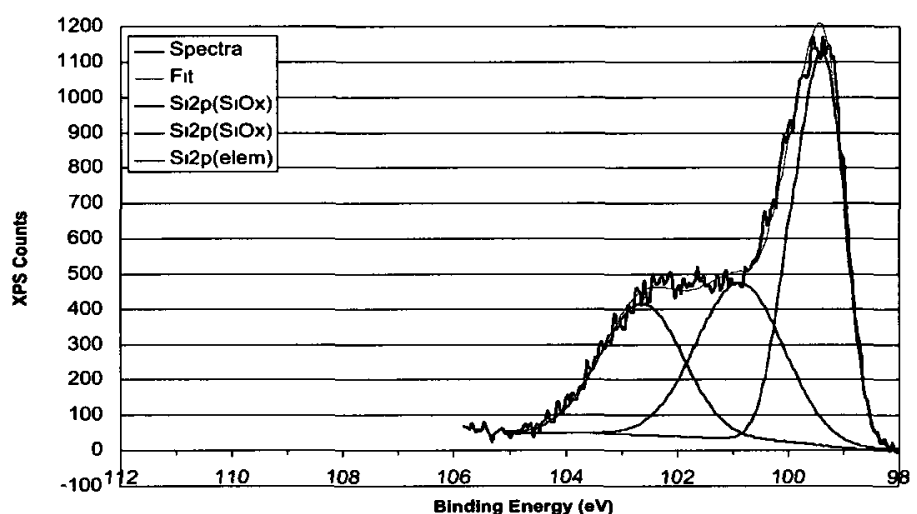

(c) After 10 min of Ar sputtering

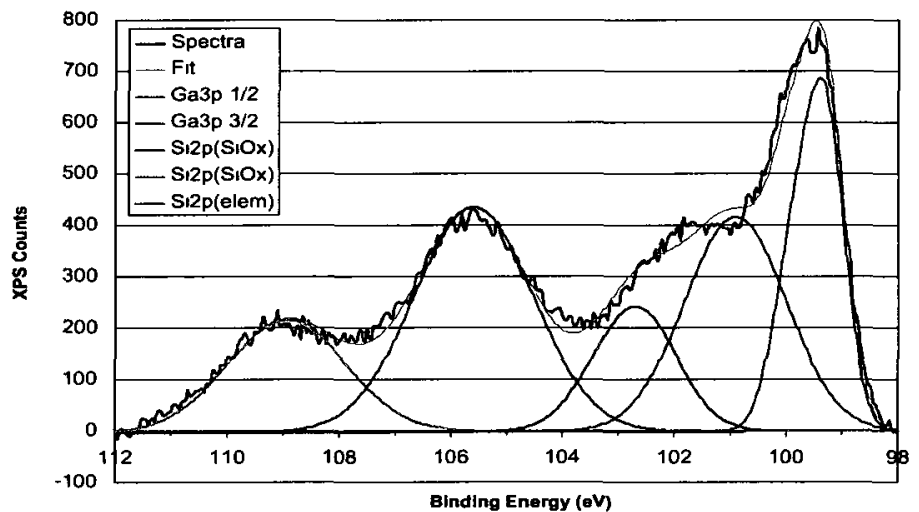

(e) After 30 min of Ar sputtering

\begin{tabular}{lccc}
\hline Peak & $\begin{array}{c}\text { Position } \\
\text { eV }\end{array}$ & $\begin{array}{c}\text { Area } \\
\text { FWHM } \\
\text { eV }\end{array}$ \\
\hline $\mathrm{S} 12 \mathrm{p}\left(\mathrm{SiO}_{2}\right)$ & 1032 & 9899 & 140 \\
$\mathrm{~S} 2 \mathrm{p}\left(\mathrm{SlO}_{x}\right)$ & 1012 & 5273 & 308 \\
$\mathrm{~S} 2 \mathrm{p}($ elem $)$ & 993 & 11754 & 085 \\
\hline
\end{tabular}

(b) Before Ar sputtering peak parameters

\begin{tabular}{lccc}
\hline Peak & $\begin{array}{c}\text { Position } \\
\text { eV }\end{array}$ & Area & $\begin{array}{c}\text { FWHM } \\
\text { eV }\end{array}$ \\
\hline $\mathrm{S} 12 \mathrm{p}\left(\mathrm{S}_{1} \mathrm{O}_{x} \mathrm{I}\right)$ & 1024 & 6867 & 160 \\
$\mathrm{~S} 12 \mathrm{p}\left(\mathrm{SiO}_{x} \mathrm{II}\right)$ & 1009 & 8674 & 184 \\
$\mathrm{~S} 12 \mathrm{p}($ elem $)$ & 993 & 13076 & 085 \\
\hline \hline
\end{tabular}

(d) After 10 min of Ar sputtering peak parameters

\begin{tabular}{|c|c|c|c|}
\hline Peak & $\begin{array}{c}\text { Position } \\
\text { eV }\end{array}$ & Area & $\begin{array}{c}\text { FWHM } \\
\text { eV }\end{array}$ \\
\hline Ga3p 1/2(AlGaN) & 1089 & 5792 & 249 \\
\hline Ga3p $3 / 2(\mathrm{AlGaN})$ & 1056 & 11584 & 249 \\
\hline $\mathrm{S}_{1} 2 \mathrm{p}\left(\mathrm{S}_{1} \mathrm{O}_{r} \mathrm{I}\right)$ & 1025 & 4497 & 160 \\
\hline $\mathrm{S} 12 \mathrm{p}\left(\mathrm{SlO}_{x} \mathrm{II}\right)$ & 1009 & 9516 & 215 \\
\hline $\mathrm{S} 12 \mathrm{p}(\mathrm{elem})$ & 993 & 8072 & 085 \\
\hline
\end{tabular}

(f) After 30 min of Ar sputtering peak parameters

Figure 4.22: Batch-2 R2 XPS spectra before, after 10 and $30 \mathrm{~min}$ of Ar sputtering. 
A Si $2 p$ peak was observed on Si-treated and wet etched samples that did not belong to either $\mathrm{SiO}_{2}$ or elemental $\mathrm{Si}$ (Fig. 4.23(a)). Because of the aggressiveness of $\mathrm{HF}: \mathrm{HNO}_{3}$ wet etch and based on the location of the peak [77] (Fig. 4.24), it was concluded that this peak was associated with a non-stoichiometric $\operatorname{SiN}_{x}$ containing surface film. Fig. 4.24 Additionally, the ohmic contact processing steps resulted in an additional peak corresponding to $\mathrm{SiO}_{2}(103.4 \mathrm{eV})$, indicating that the film was easily oxidized (Fig. 4.23(c)).

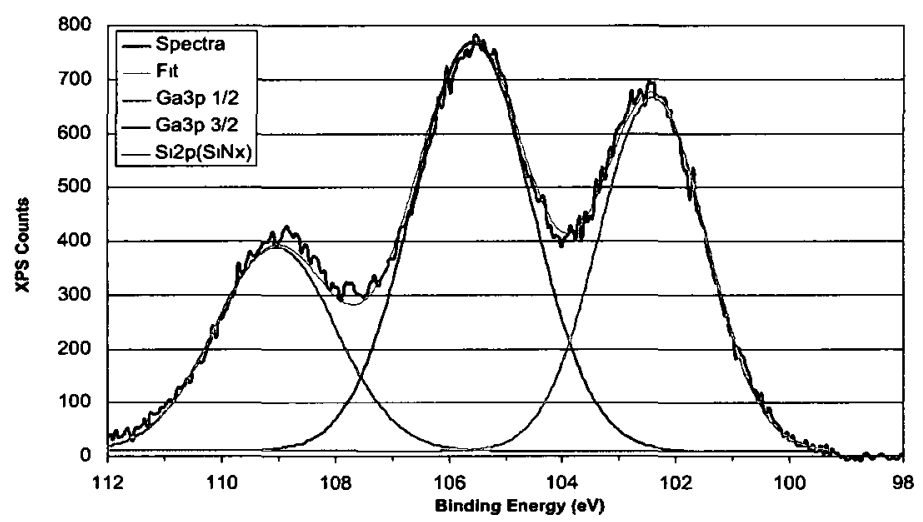

(a) Remnant $\mathrm{SiN}_{x}$ film on the sample $\mathrm{S} 2$ after wet etch

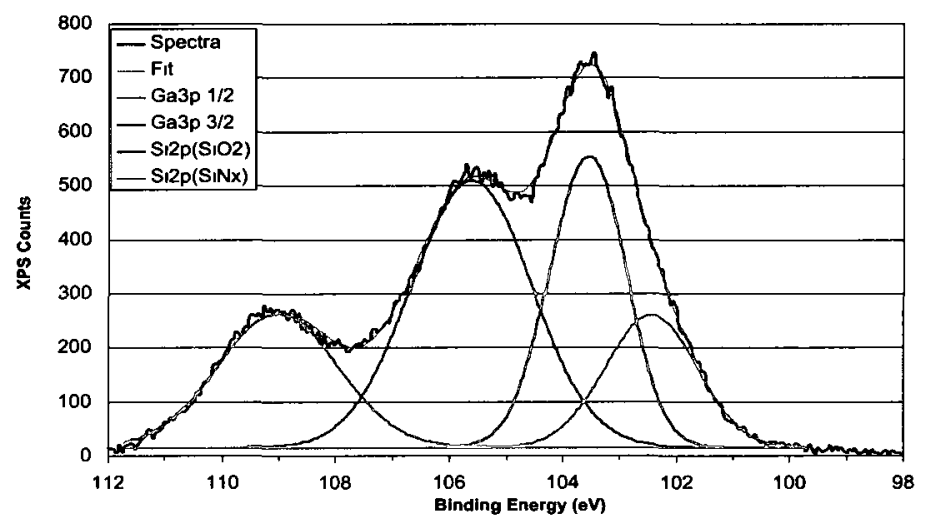

(c) Remnant $\mathrm{SiN}_{x} / \mathrm{SiO}_{2}$ film on the sample $\mathrm{S} 2$ after processing steps leading to ohmic contact metal deposition

\begin{tabular}{lccc}
\hline Peak & $\begin{array}{c}\text { Position } \\
\text { eV }\end{array}$ & Area & $\begin{array}{c}\text { FWHM } \\
\text { eV }\end{array}$ \\
\hline Ga3p 1/2(AlGaN) & 1091 & 10053 & 249 \\
Ga3p 3/2(AlGaN) & 1056 & 20105 & 249 \\
S12p(SıN & 1022 & 15608 & 212 \\
\hline \hline
\end{tabular}

(b) Remnant $\mathrm{SiN}_{x}$ peak parameters

\begin{tabular}{lccc}
\hline \hline Peak & $\begin{array}{c}\text { Position } \\
\text { eV }\end{array}$ & Area & $\begin{array}{c}\text { FWHM } \\
\text { eV }\end{array}$ \\
\hline Ga3p 1/2(AlGaN) & 1090 & 6554 & 249 \\
Ga3p 3/2(AlGaN) & 1056 & 13107 & 249 \\
Si2p $\left(\mathrm{SiO}_{2}\right)$ & 1034 & 9019 & 141 \\
$\mathrm{~S} 2 \mathrm{p}\left(\mathrm{S}_{1} \mathrm{~N}_{r}\right)$ & 1022 & 4932 & 177 \\
\hline
\end{tabular}

(d) Remnant $\mathrm{SiN}_{x} / \mathrm{SiO}_{2}$ peak parameters

Figure 4.23: Batch-3 S2 XPS spectra before and after processing steps leading to ohmic contact metal deposition. 


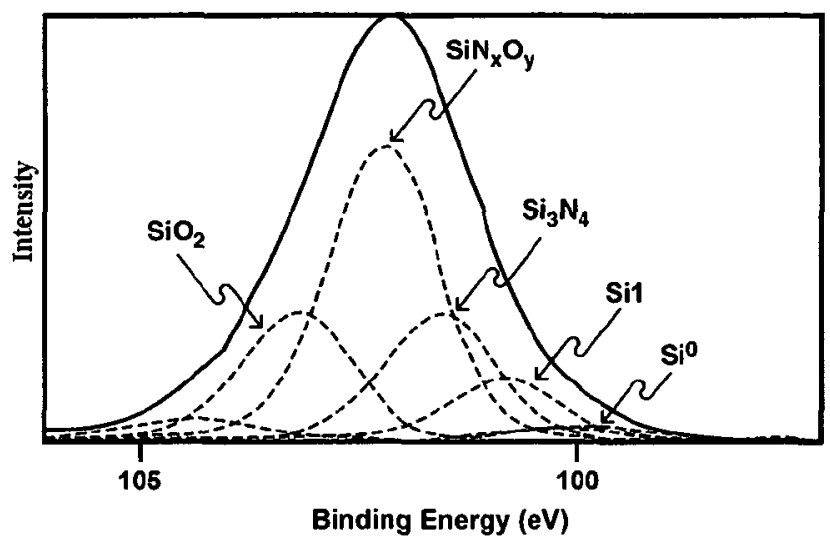

Figure 4.24: The location of several Si peaks from peak fitting by Matsuoka et al. $[77]$.

The AR-XPS surface composition measurement was performed on sample S2 from Batch-3 after the water contact angle measurement. The spectra recorded for take off angles of 30, 45 and 77.5 degrees are shown in Fig. 4.25. Assuming that most of the $\mathrm{SiN}_{x}$ was consumed and using a photoelectric attenuation length of $3 \mathrm{~nm}$ for $\mathrm{SiO}_{2}$ film [51], $\beta_{S \imath / G a}$ for $2 p$ and $3 p$ peaks of $0.27 / 0.84=0.32$ [78], a fit between the AR-XPS equation (Eq. 2.45) and the experimental data was obtained (Fig. 4.26). The best fit resulted in a film thickness of $2.45 \mathrm{~nm}$, in agreement with SIMS results.

An additional way to estimate the thickness of the oxidized $\operatorname{SiN}_{x}$ film is to compare its peak intensity to the $\mathrm{Si}$ peak intensity of the native $\mathrm{SiO}_{2}$ film on the sample $\mathrm{R} 2$ from Batch-2. The combined areas of Si peaks produced by remnant film $\left(\mathrm{SiN}_{x}\right.$ and $\left.\mathrm{SiO}_{2}\right)$ and native $\mathrm{SiO}_{2}$ were $(902+493)$ and 990 respectively. Given a native oxide thickness of $1.5-2.0 \mathrm{~nm}$, the estimate of the remnant $\mathrm{SiN}_{x}$ film thickness was 2.1 $2.8 \mathrm{~nm}$. The oxidation had likely increased the overall thickness of the film, thus the original $\mathrm{SiN}_{x}$ film was thinner.

Film thickness obtained from SIMS and AR-XPS measurements as well as from the comparison to the native oxide peak agreed. A rough final estimate of the initial (non-oxidized) $\mathrm{SiN}_{x}$ film thickness was $1.0-2.0 \mathrm{~nm}$. 


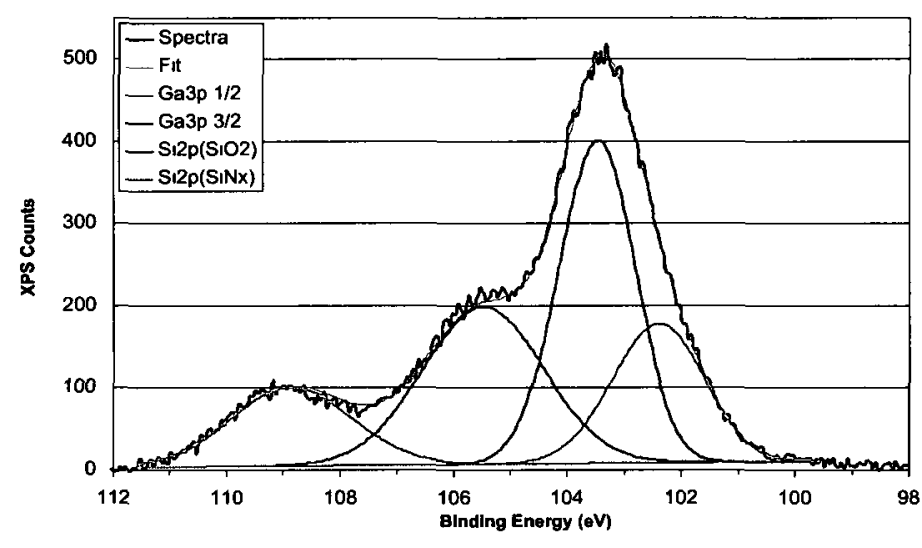

(a) Spectra at $30^{\circ}$ take off angle

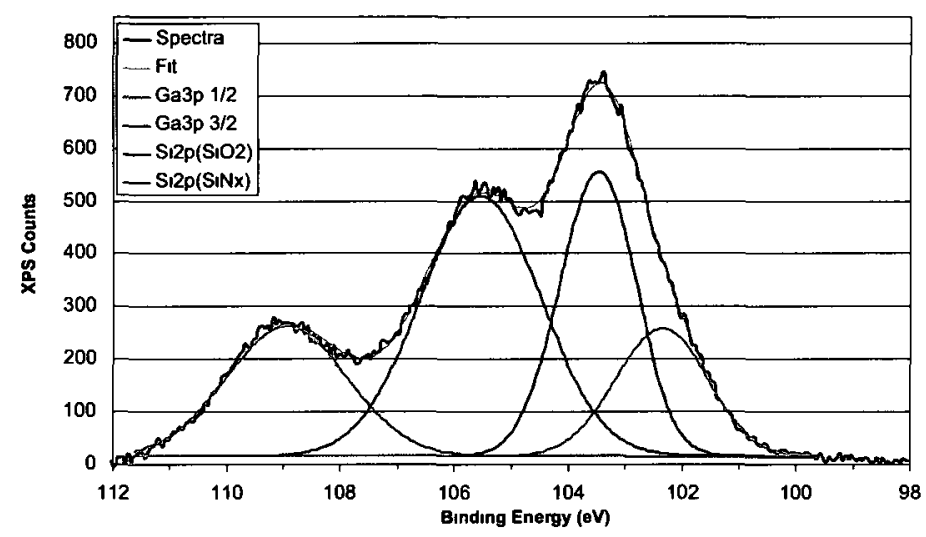

(c) Spectra at $45^{\circ}$ take off angle

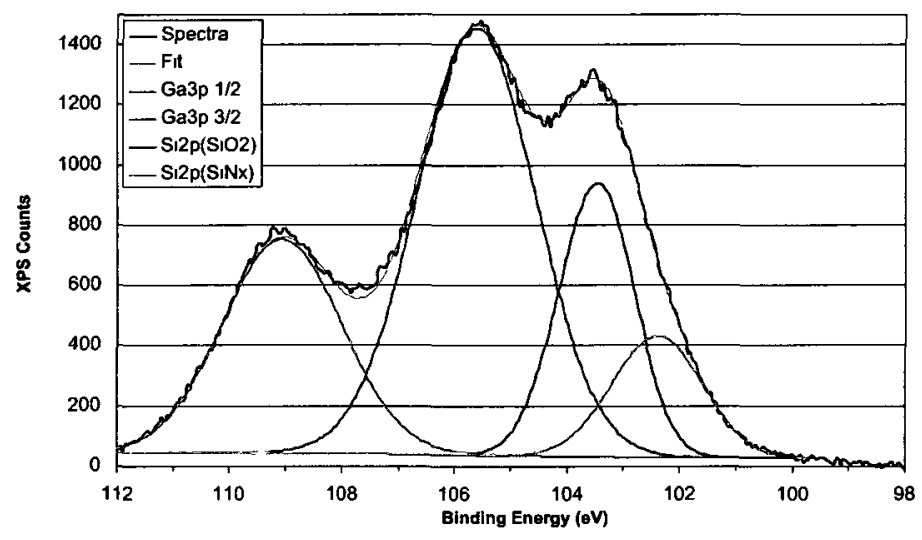

(e) Spectra at $77.5^{\circ}$ take off angle

\begin{tabular}{lccc}
\hline \hline Peak & $\begin{array}{c}\text { Position } \\
\text { eV }\end{array}$ & $\begin{array}{c}\text { Area } \\
\text { FWHM } \\
\text { eV }\end{array}$ \\
\hline Ga3p(AlGaN) & 1089 & 2542 & 249 \\
$\mathrm{Ga3p}(\mathrm{AlGaN})$ & 1055 & 5084 & 249 \\
$\mathrm{~S} 2 \mathrm{p}\left(\mathrm{S}_{1} \mathrm{O}_{2}\right)$ & 1033 & 6577 & 141 \\
$\mathrm{~S} 12 \mathrm{p}\left(\mathrm{S}_{1} \mathrm{~N}_{x}\right)$ & 1022 & 3407 & 177 \\
\hline \hline
\end{tabular}

(b) $30^{\circ}$ peak parameters

\begin{tabular}{lccc}
\hline \hline Peak & $\begin{array}{c}\text { Position } \\
\text { eV }\end{array}$ & Area & $\begin{array}{c}\text { FWHM } \\
\text { eV }\end{array}$ \\
\hline $\mathrm{Ga3p}(\mathrm{AlGaN})$ & 1089 & 6554 & 249 \\
$\mathrm{Ga3p}(\mathrm{AlGaN})$ & 1055 & 13107 & 249 \\
$\mathrm{~S} 2 \mathrm{p}\left(\mathrm{S}_{1} \mathrm{O}_{2}\right)$ & 1033 & 9050 & 141 \\
$\mathrm{~S} 2 \mathrm{p}\left(\mathrm{SiN}_{x}\right)$ & 1022 & 4902 & 177 \\
\hline \hline
\end{tabular}

(d) $45^{\circ}$ peak parameters

\begin{tabular}{lccc}
\hline Peak & $\begin{array}{c}\text { Position } \\
\text { eV }\end{array}$ & Area & $\begin{array}{c}\text { FWHM } \\
\text { eV }\end{array}$ \\
\hline Ga3p(AlGaN) & 1090 & 18814 & 249 \\
Ga3p $(\mathrm{AlGaN})$ & 1056 & 37628 & 249 \\
$\mathrm{~S} 2 \mathrm{p}\left(\mathrm{SIO}_{2}\right)$ & 1033 & 15198 & 141 \\
$\mathrm{~S} 2 \mathrm{p}\left(\mathrm{SLN}_{x}\right)$ & 1022 & 8133 & 177 \\
\hline \hline
\end{tabular}

(f) $77.5^{\circ}$ peak parameters

Figure 4.25: Batch-3 S2 AR-XPS spectra at $30^{\circ}, 45^{\circ}$ and $77.5^{\circ}$ take off angle. 


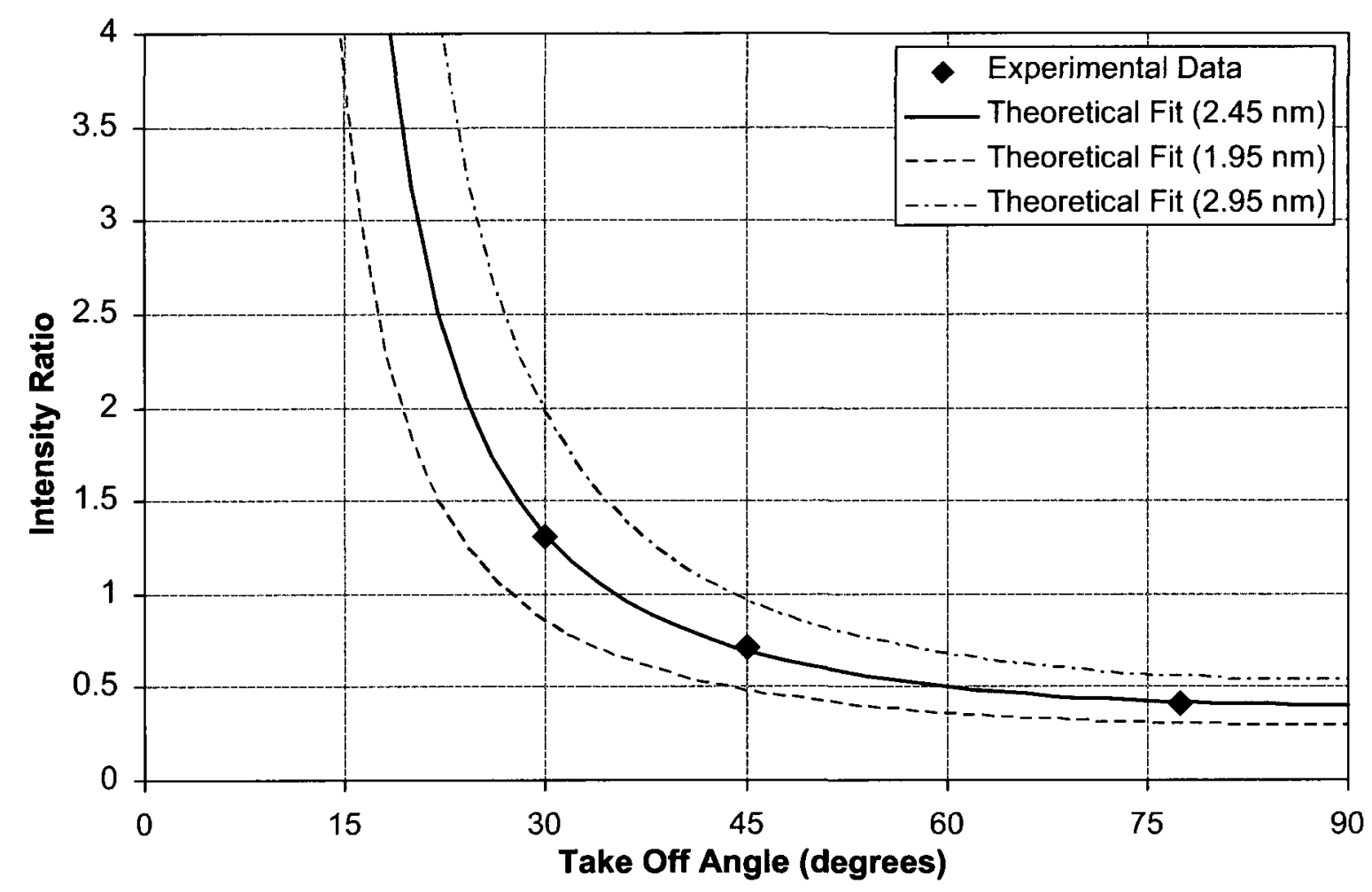

Figure 4.26: The fit of the AR-XPS equation to the experimental data using Si to Ga sensitivity ratio parameter $\beta$ of 0.32 , photoelectron attenuation length $\lambda$ of $3 \mathrm{~nm}$ and the film thickness $d$ of $2.45 \mathrm{~nm}$. Theoretical curves with the values of $d=1.95 \mathrm{~nm}$ and $d=2.95 \mathrm{~nm}$ are also shown for comparison. 
The combined plot of XPS spectra for several samples from Batch-2 and Batch3 is shown in Fig. 4.27. The oxidation of the remnant $\mathrm{Si}$ film at several stages of processing can be clearly seen.

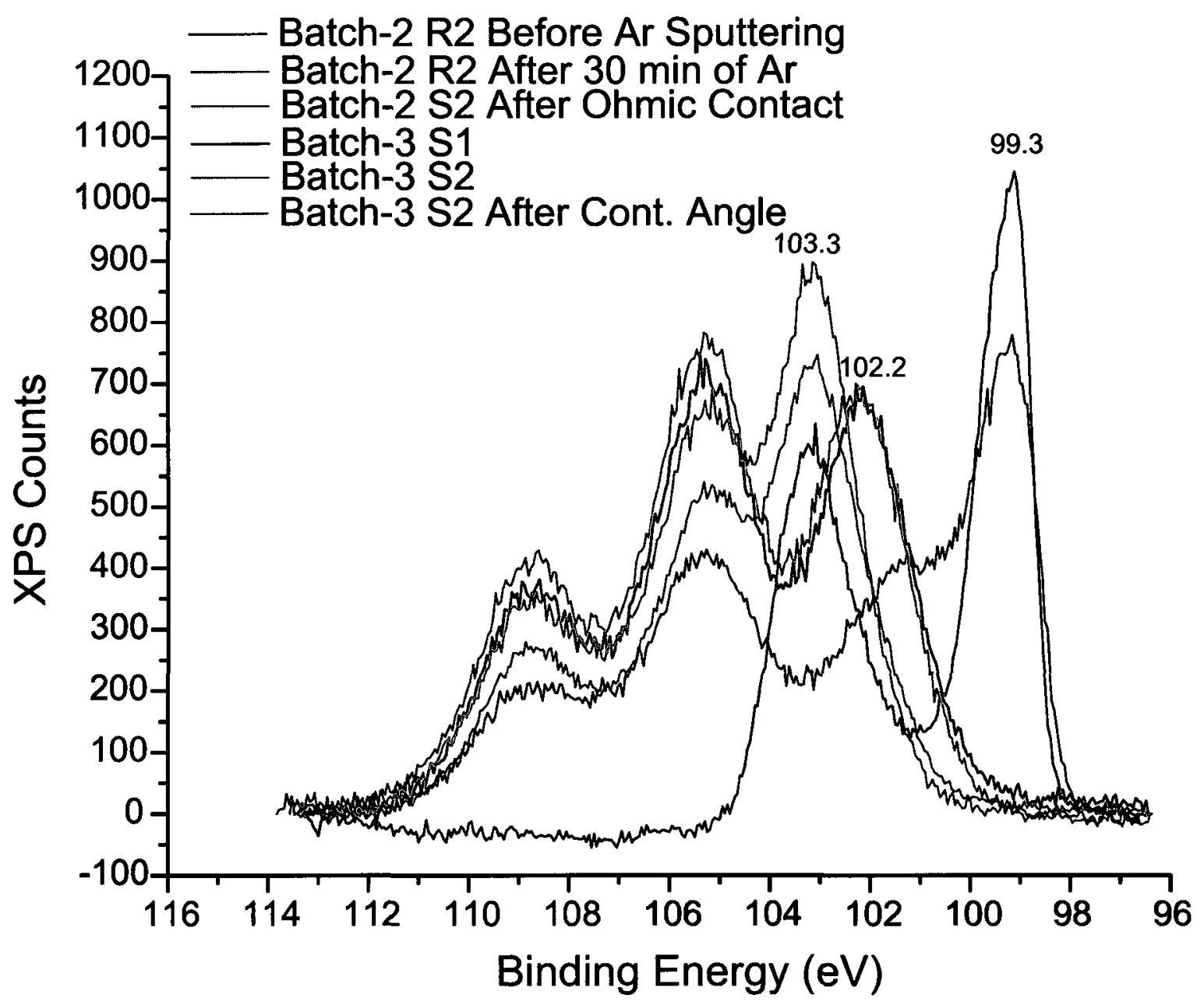

Figure 4.27: Combined plot of XPS spectra for several samples from Batch-2 and Batch-3 


\subsection{AlGaN Surface Water Contact Angle}

The similarity between Si-coated and Si-treated samples is clearly seen from the water contact angle measurement results (Fig. 4.28). Hydrophilic surfaces are characteristic to oxides and Si-treated and coated samples were expected to grow oxides of $\mathrm{Si}$ in the top most layers. This measurement clearly distinguishes the control samples from the Si-treated or coated samples, which further signified the presence of the remnant Si-containing film on the Si-treated samples S1 and S2.

The difference in contact angles between the control samples and Si-treated/coated samples at each of the last three processing steps have very significant implications. Firstly, there was already a contact angle difference of $15^{\circ}$ following the "pre ohmic cleaning". Thus the actual clean could be affected by the hydrophilic properties of the surface at that step. Moreover, following Deionized (DI) water rinse, the contact angle difference exceeded $20^{\circ}$. The additional 20 hours of storage time resulted in the contact angle of about $45^{\circ}$ on control samples while the angle remained at about $5^{\circ}$ on Si-treated and coated samples. The time period between "pre ohmic clean" and ohmic metal evaporation can be crucial for control samples, affecting the reproducibility of the ohmic contact resistance values. Si-treated and Si-coated AlGaN surfaces might be insensitive to the time between these steps, based on the fairly constant water contact angle value displayed by these samples. 


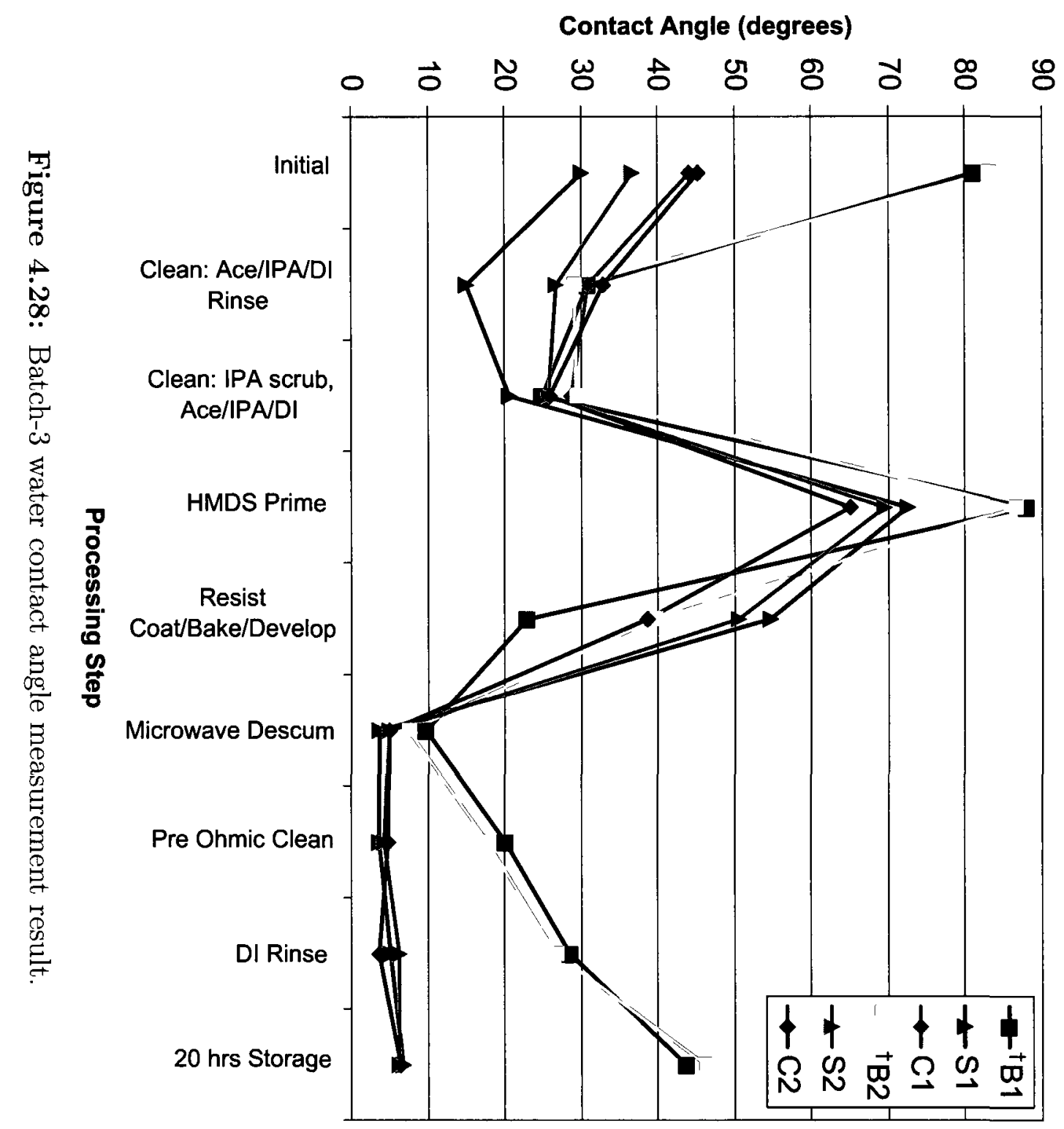




\section{Chapter 5}

\section{DISCUSSION}

The rigorous investigation of the AlGaN surface treatments and coatings with $\mathrm{Si}$ films revealed several important findings which were not previously reported in the literature. The results obtained from SIMS profiling, XPS surface composition and contact angle measurements on the samples following Si film deposition, RTA annealing and film removal, clearly indicated that a remnant Si-containing film was left on the surface. Given the aggressiveness of the $\mathrm{HF}: \mathrm{HNO}_{3}$ wet etch towards both $\mathrm{Si}$ and $\mathrm{SiO}_{2}$, elemental or oxidized $\mathrm{Si}$ were ruled out as the candidates for the composition of the remnant film. The location of the Si $2 p$ peak on the Si-treated samples in XPS spectrum further confirmed that the Si in the film did not belong to either one of those states but belonged to a non-stoichiometric $\mathrm{SiN}_{x}$ compound. The formation of this $\operatorname{SiN}_{x}$ compound under high temperature annealing is easily justified when the enthalpy of formations are examined. The enthalpy of formation of $\mathrm{Si}_{3} \mathrm{~N}_{4}$ is $-850 \mathrm{~kJ} / \mathrm{mol}[79]$ and $\mathrm{TiN}$ is $-265 \mathrm{~kJ} / \mathrm{mol}$. Since there is overwhelming proof of Ti forming TiN compounds during ohmic contact annealing, $\mathrm{Si}_{3} \mathrm{~N}_{4}$ should be easily formed given its lower enthalpy of formation, especially considering high annealing temperatures used in this study. The film was found to be easily oxidized upon further processing. The low stability of the film was attributed to its non-stoichiometric structure and enthalpy of formation for $\mathrm{SiO}_{2},-911 \mathrm{~kJ} / \mathrm{mol}$, lower than for $\mathrm{Si}_{3} \mathrm{~N}_{4}$. The 
thickness of the remnant film (prior to any oxidation) was estimated to be between 1.0 and $2.0 \mathrm{~nm}$ from both XPS and SIMS measurement results.

The inclusion of the Si-coated reference sample clearly revealed the SIMS measurement artifact on Si-treated samples. Without the reference, the shape of the $\mathrm{Si}$ profile in the SIMS measurements might be attributed to the thermal diffusion of $\mathrm{Si}$. However, by comparing the profiles of Si-treated samples to the reference sample it can be seen that the solid state diffusion, if any, was heavily masked by the measurement artifact arising from the interaction of a primary ion beam and the remnant Si-containing film.

The discovery of this remnant film resulting from the reaction between $\mathrm{Si}$ and AlGaN layers opens new avenues to understanding how the annealing of the $\mathrm{Si}$ thin film on AlGaN surfaces, which was thought to result in solid state diffusion, can improve or alter electrical performance of fabricated devices. It is known that $\mathrm{Si}$ acts as a bulk n-type dopant in the AlGaN layer. It has also been suggested that Si acts as a donor on the AlGaN surface providing passivating results similar to those achieved with $\mathrm{Si}_{3} \mathrm{~N}_{4}[69,71]$. Since the devices on Si-coated samples produced similar, if not better, results in the current collapse and current density measurements, diffusion of Si into the epilayer as a dominant mechanism for electrical enhancements appears unlikely. The evidence gathered here indicates that $\mathrm{Si}$ acts as a shallow surface sheet donor, providing the electrons to the 2DEG and/or to surface electron traps. This in turn reduced the current collapse and improved the 2DEG densities. However, the actual mechanism by which $\mathrm{Si}$ acts as a surface donor is still not clear.

In a fashion similar to the formation of the tunnelling ohmic contacts in which the reaction of titanium and nitrogen creates an AlGaN surface heavily n-type doped with nitrogen vacancies, the reaction between $\mathrm{Si}$ and $\mathrm{AlGaN}$, resulting in $\mathrm{SiN}_{x}$ formation, might also produce a surface heavily n-type doped with nitrogen vacancies. Given the temperatures at which TiN forms during ohmic contact metal alloying, the reaction 
between $\mathrm{Si}$ and $\mathrm{AlGaN}$ should readily progress at temperatures in the range of 800 to $1,000^{\circ} \mathrm{C}$. Based on enthalpy of formation, $\mathrm{Si}$ and $\mathrm{AlGaN}$ materials should react more strongly than Ti and AlGaN, resulting in a heavier doping of the AlGaN surface.

The water contact angle measurement has revealed the similarity between Sicoated and Si-treated samples, and the results were in sharp contrast to the measurements on the control samples. Combined with the XPS and SIMS measurements, this finding is also explained by the remnant $\mathrm{SiN}_{x} / \mathrm{SiO}_{x}$ film left on the AlGaN surface. Of particular interest are the results after the last few processing steps leading to the ohmic metal deposition. There is a clear change in the contact angle on the control samples. The water contact angle remains fairly constant on Si-treated and coated samples after the pre ohmic metal clean, followed by the DI rinse and 20 hours of storage time, in contrast to the contact angle on the control samples. This result has two implications. First, reproducibility is much easier achieved on Si-treated and coated samples given that the hydrophilic properties of their surfaces do not change significantly with time. Secondly, stronger surface hydrophilic properties can enhance the cleaning of the surface resulting in better removal of common contaminants.

The ohmic contact resistances were not as sensitive to high alloying temperatures on the Si-treated and coated samples. The remnant Si may thus require slightly higher temperatures to become an active part of the complete ohmic contact or it may slow down the rate of certain reactions occurring during high annealing temperatures. Sitreated and coated samples demonstrated far superior characteristics than the control sample upon heavy oxidation of the ohmic metal in Batch-2. These results indicate that $\mathrm{Si}$ treatments and coatings can improve the ohmic contacts and device current densities in unfavourable processing conditions for the ohmic contact metal. Given that NRC's processing for ohmic contacts is very well optimized for the material used in this study, it may explain why other groups witnessed substantial improvements to their ohmic contacts. 
Under proper processing conditions similar ohmic contact resistances were obtained on both Si-treated, coated and control samples, indicating that the remnant film was not providing any significant improvements to the ohmic contacts. The lowest ohmic contact resistances achieved in this study are in the range of 0.5 to 0.6 Ohm-mm. Assuming the 2DEG sheet resistance under the ohmic contact $\left(R_{s c}\right)$ is equal to the 2DEG sheet resistance on unpassivated material $R_{s}(\sim 400 \mathrm{Ohm} / \mathrm{sq})$, a specific contact resistance in the range of $6.3 \times 10^{-6}$ to $9.0 \times 10^{-6} \mathrm{Ohm}-\mathrm{cm}^{2}$ was obtained using Eq. 2.39 and Eq. 2.41.

The passivating properties of the Si treatment were seen in the Hall measurements as well as in the 2DEG sheet resistance measurements on TLM and HEMT devices. Si-treated and Si-coated surfaces were found to be pre-passivated before the SiN passivation step, which is beneficial for in-process device performance monitoring and adjustments. The HEMTs exhibited noticeably lower current collapse and higher saturation current densities on both Si-treated (Batch-1) and Si-coated (Batch-3) samples before the passivation. In addition, there was evidence that Si treatments and coatings improve the 2DEG densities even after the SiN passivation. The 2DEG sheet resistances were noticeably better on Si-treated samples in Batch-1 and HEMTs had higher saturation current densities on both Si-treated and Si-coated samples from Batch-1 and Batch-3. The peak saturation current densities for $V_{g}=2 \mathrm{~V}$ reached values of $915 \mathrm{~mA} / \mathrm{mm}$ on the passivated control sample, $1,050 \mathrm{~mA} / \mathrm{mm}$ on the Sitreated sample and 1,090 $\mathrm{mA} / \mathrm{mm}$ on the Si-coated sample in Batch-3. These results are in agreement with findings by Onojima et al. [69], where Si films were used for passivation. Similar current enhancements to the current densities were obtained: from $1,100 \mathrm{~mA} / \mathrm{mm}$ to $1,200 \mathrm{~mA} / \mathrm{mm}$.

Several electrical measurements revealed high gate and drain leakage currents on Si-treated and coated samples. Gate Schottky junction ideality factors were significantly lower for Si-treated samples, indicating that the thermionic emission became 
a more dominant mechanism of current conduction. This result is further supported by higher reverse bias leakage currents obtained on Si-treated samples. In addition, the shape of the reverse bias I-V curve for Si-treated samples were significantly flatter, indicating a more ideal Schottky junction behaviour conforming better to the ideal diode equation. Higher leakages and the dominant thermionic current transport mechanism are consistent with the lowering of the Schottky barrier height, as can be seen in Eq. 2.28. Higher leakage currents on Si-treated and Si-coated samples can also be explained by an additional leakage path on top of the AlGaN epilayer. This leakages may have lead to filling of surface states that passivate the surface thereby increasing 2DEG densities in the channel. The magnitude of the leakages was in the range of a few $\mathrm{mA} / \mathrm{mm}$, not sufficient to account for the improvements in 2DEG sheet resistance values or HEMT peak drain current densities following the AlGaN surface treatment or coating with $\mathrm{Si}$.

There was a distinct change in threshold voltage in fabricated devices on Sitreated and Si-coated samples. This shift, which is also observed in metal-insulatorsemiconductor HEMT devices where an insulator is placed under the gate, can be explained by the presence of remnant $\mathrm{SiN}_{x}$ and $\mathrm{Si}$ films on the AlGaN surface.

The experiments did not reveal any significant improvements to AlGaN/GaN device performance following the surface treatment with SiON films. Therefore it was concluded that the SiON films were intentionally left in place for further processing in the previous studies. The benefits of using $\mathrm{SiON}$ instead of $\mathrm{Si}$ could come from the ease with which the film can be wet etched in comparison to $\mathrm{Si}$ or $\mathrm{Si}_{3} \mathrm{~N}_{4}$. However, the leakage currents were significantly reduced on SiON-treated samples as well as otherwise untreated RTA annealed samples. The formation of very thin oxide films on AlGaN surface is thought to be responsible for these results.

A few disadvantages of Si treatments and coatings of AlGaN surfaces are longer 
process flows, the requirement of better quality control of $\mathrm{Si}$ deposition (as demonstrated by the cracking of the ohmic metal in Batch-2 and Batch-3 samples) and higher leakage currents in fabricated devices. 


\section{Chapter 6}

\section{CONCLUSIONS AND FUTURE WORK}

\subsection{Conclusion}

In conclusion, the effects of Si treatments and coatings on AlGaN surfaces were rigorously studied. Several important findings were made and presented. Si treatment resulted in a remnant $1.0-2.0 \mathrm{~nm}$ thick $\mathrm{SiN}_{x}$ film left on the AlGaN surface. SIMS measurement artifact due to this film was demonstrated. The water contact angle measurement provided an additional evidence for the difference between the Si-treated, Si-coated and control samples. In addition, the contact angles varied substantially on the control samples in sharp contrast to the contact angle on the treated and coated surfaces. The electrical changes in fabricated devices were attributed to the remnant or intentionally left Si film, not the solid state diffusion. The electrical measurement results demonstrated pre-passivating properties of Si treatments and coatings. In addition, Si treatment and coatings produced better ohmic contact resistances in adverse processing conditions. Under proper processing conditions similar

ohmic contact resistance values of about $0.5-0.6 \mathrm{Ohm}-\mathrm{mm}$ were obtained on treated, coated and control samples. Si-treated and coated samples displayed higher leakage currents and more negative threshold voltages. 


\subsection{Future Work}

This study did not reveal how exactly $\mathrm{Si}$ acted as a surface sheet donor. The parameters such as a donor energy level and density may be established by measuring the 2DEG channel resistance and carrier density versus temperature in a cryostat. Additionally, performing the Si treatments and coatings using thinner AlGaN epilayers might result in more drastic enhancements, revealing changes to the device performance undetected in this study.

Another area for future work lies in the determination of Schottky barrier heights and ideality factors in Si-treated and Si-coated samples. Although this study suggests that the Schottky barrier height is reduced on Si-treated and Si-coated samples, the evidence is neither overwhelming nor complete.

In order to successfully use the $\mathrm{Si}$ treatments and coatings in production, the problem with cracking of the ohmic metal stack has to be resolved. Because this effect was not noticed during the first fabrication run, it might be possible to fully eliminate it. One possible way to address this problem is to evaporate Si in-situ prior to ohmic contact deposition. 


\section{List of References}

[1] U. K. Mishra, L. Shen, T. E. Kazior, and Y. Wu, "GaN-based RF power devices and amplifiers," Proceedings of the IEEE, vol. 96, no. 2, pp. 287-305, 2008.

[2] O. Ambacher, "Growth and applications of group III-nitrides," Journal of Physıcs D: Applied Physıcs, vol. 31, no. 20, pp. 2653-2710, 1998.

[3] T. Nomura, M. Masuda, N. Ikeda, and S. Yoshida, "Switching characteristics of GaN HFETs in a half bridge package for high temperature applications," IEEE Transactıons on Power Electronıcs, vol. 23, no. 2, pp. 692-697, 2008.

[4] S. J. Pearton, C. R. Abernathy, and F. Ren, Galluum Nitrude Processing for Electronıcs, Sensors and Spıntronıcs. London, England: Springer-Verlag, 2006.

[5] R. Quay, Gallıum Nitride Electronıcs. Berlin, Germany: Springer, 2008.

[6] P. Jonnard, N. Capron, F. Semond, J. Massies, E. Martinez-Guerrero, and H. Mariette, "Electronic structure of wurtzite and zinc-blende AlN," The European Physical Journal B, vol 42, pp. 351-359, 2004.

[7] "V semiconductors optics and optoelectronics I." http://kottan-labs.bgsu.edu/ teachıng/workshop2001/chapter5.htm, 2001.

[8] S. C. Jain, M. Willander, J. Narayan, and R. Van Overstraeten, "III-nitrides: Growth, characterization, and properties," Journal of Applied Physıcs, vol. 87, no. 3 , pp. 965-1006, 2000.

[9] H. T. Wang, L. S. Tan, and E. F. Chor, "Optical and electrical characterization of annealed silicon-implanted GaN," Semıconductor Scence and Technology, vol. 19, no. 2, pp. 142-146, 2004.

[10] J. D. Plummer, M. D. Deal, and P. B. Griffin, Stlıcon VLSI Technology. Upper Saddle River, NJ USA: Prentice Hall Inc, 2000. 
[11] X. A. Cao, S. J. Pearton, R. K. Singh, C. R. Abernathy, J. Han, R. J. Shul, D. J. Rieger, J. C. Zolper, R. G. Wilson, M. Fu, J. A. Sekhar, H. J. Guo, and S. J. Pennycook, "Rapid thermal processing of implanted GaN up to $1500{ }^{\circ} \mathrm{C}$," Materıals Research Socıety Symposıum - Proceedıngs, vol. 537, 1999.

[12] Y. Mishin and C. Herzig, "Grain boundary diffusion: Recent progress and future research," Materıals Scıence and Engineering A, vol. 260, no. 1-2, pp. 55-71, 1999.

[13] R. Jakiela, A. Barcz, E Dumiszewska, and A. Jagoda, "Si diffusion in epitaxial GaN," Physıca Status Solıd (C) Current Topıcs in Solıd State Physıcs, vol. 3, pp. 1416-1419, 2006.

[14] P. H. Ladbrooke, MMIC Design: GaAs FETs and HEMTs. Norwood, MA USA: Artech house Inc, 1989.

[15] S. M. Sze, High-Speed Semiconductor Devices. USA: John Wiley \& Sons Inc, 1990.

[16] M. Asif Khan, J. N. Kuznia, A. R. Bhattarai, and D. T. Olson, "Metal semiconductor field effect transistor based on single crystal GaN," Appleed Physıcs Letters, vol. 62, no. 15, pp. 1786-1787, 1993.

[17] M. Asif Khan, A. Bhattarai, J. N. Kuznia, and D. T. Olson, "High electron mobility transistor based on a GaN-AlGaN heterojunction," Applzed Physics Letters, vol. 63, no. 9, pp. 1214-1215, 1993.

[18] J. A. Bardwell, T. Lester, H. Tang, B. Surridge, A. Harrison, and A. Lau, "AlGaN/GaN transistors and circuits." Power Point Presentation, 2009.

[19] F. Bernardini, V. Fiorentini, and D. Vanderbilt, "Spontaneous polarization and piezoelectric constants of III-V nitrides," Physıcal Revıew B - Condensed Matter and Materals Physics, vol 56, no. 16, 1997.

[20] O. Ambacher, B. Foutz, J. Smart, J. R. Shealy, N. G. Weimann, K. Chu, M. Murphy, A. J. Sierakowski, W. J. Schaff, L. F. Eastman, R. Dimitrov, A. Mitchell, and M. Stutzmann, "Two dimensional electron gases induced by spontaneous and piezoelectric polarization in undoped and doped AlGaN/GaN heterostructures," Journal of Applzed Physics, vol. 87, no. 1, pp. 334-344, 2000.

[21] O. Ambacher, J. Majewski, C. Miskys, A. Link, M. Hermann, M. Eickhoff, M. Stutzmann, F. Bernardini, V. Fiorentini, V. Tilak, B. Schaff, and L. F. 
Eastman, "Pyroelectric properties of $\mathrm{Al}(\mathrm{In}) \mathrm{GaN} / \mathrm{GaN}$ hetero- and quantum well structures," Journal of Physics Condensed Matter, vol. 14, no. 13, pp. 3399-3434, 2002.

[22] N. Li, D. G. Zhao, and H. Yang, "Polarization effects simulation of AlGaN/GaN heterojunction by using a symbolistic $\delta$-doping layer," Solıd State Communıcatrons, vol. 132, no. 10, pp. 701-705, 2004.

[23] I. P. Smorchkova, C. R. Elsass, J. P. Ibbetson, R. Vetury, B. Heying, P. Fini, E. Haus, S. P. DenBaars, J. S. Speck, and U. K. Mishra, "Polarization-induced charge and electron mobility in $\mathrm{AlGaN} / \mathrm{GaN}$ heterostructures grown by plasmaassisted molecular-beam epitaxy," Journal of Applıed Physıcs, vol. 86, no. 8, pp. 4520-4526, 1999.

[24] J. P. Ibbetson, P. T. Fini, K. D. Ness, S. P. DenBaars, J. S. Speck, and U. K. Mishra, "Polarization effects, surface states, and the source of electrons in AlGaN/GaN heterostructure field effect transistors," Applıed Physıcs Letters, vol. 77 , no. 2 , pp. 250-252, 2000.

[25] L. Shen, S. Heikman, B. Moran, R. Coffie, N. . Zhang, D. Buttari, I. P. Smorchkova, S. Keller, S. P. DenBaars, and U. K. Mishra, "Algan/aln/gan highpower microwave hemt," IEEE Electron Devnce Letters, vol. 22, no. 10, pp. 457$459,2001$.

[26] T. . Yu and K. F. Brennan, "Monte carlo calculation of two-dimensional electron dynamics in GaN-AlGaN heterostructures," Journal of Applied Physıcs, vol. 91, no. 6, pp. 3730-3736, 2002.

[27] C. S. Chang and H. R. Fetterman, "An analytic model for high-electron-mobility transistors," Solvd State Electronıcs, vol. 30, no. 5, pp. 485-491, 1987.

[28] F. Roccaforte, F. Giannazzo, F. Iucolano, J. Eriksson, M. H. Weng, and V. Raineri, "Surface and interface issues in wide band gap semiconductor electronics," Applıed Surface Scıence, vol. 256, no. 19, pp. 5727-5735, 2010.

[29] N. Chaturvedi, U. Zeimer, J. Wrfl, and G. Trnkle, "Mechanism of ohmic contact formation in AlGaN/GaN high electron mobility transistors," Semıconductor Scuence and Technology, vol. 21, no. 2, pp. 175-179, 2006.

[30] S. O. Kasap, Principles of Electronıc Materials and Devices. USA: McGraw-Hill, 2006. 
[31] M. Shur, S. Rumyantsev, and M. Levinshtein, $\mathrm{S}_{\imath} \mathrm{C}$ materıals and devices. USA: World Scientific Publishing, 2006.

[32] D. Qiao, L. S. Yu, S. S. Lau, J. M. Redwing, J. Y. Lin, and H. X. Jiang, "Dependence of Ni/AlGaN schottky barrier height on Al mole fraction," Journal of Applied Physıcs, vol. 87, no. 2, pp. 801-804, 2000.

[33] E. H. Rhoderick, "Metal-semiconductor contacts," IEE Proceedings I: Solvd State and Electron Devices, vol. 129, no. 1, pp. 1-14, 1982.

[34] Z. Fan, S. N. Mohammad, W. Kim, A. Aktas, A. E. Botchkarev, K. Suzue, H. Morko, K. Duxstad, and E. E. Haller, "Ohmic contacts and Schottky barriers to n-GaN," Journal of Electronıc Materials, vol. 25, no. 11, pp. 1703-1708, 1996.

[35] S. N. Mohammad, "Contact mechanisms and design principles for nonalloyed ohmic contacts to n-GaN," Journal of Applied Physıcs, vol. 95, no. 9, pp. 4856$4865,2004$.

[36] N. Stavitski, M. J. H. Van Dal, R. A. M. Wolters, A. Y. Kovalgin, and J. Schmitz, "Specific contact resistance measurements of metal-semiconductor junctions," vol. 2006 of International Conference on Microelectronuc Test Structures, pp. 13$17,2006$.

[37] G. S. Marlow and M. B. Das, "The effects of contact size and non-zero metal resistance on the determination of specific contact resistance," Solvd State Electronıcs, vol. 25, no. 2, pp. 91-94, 1982.

[38] S. Ruvimov, Z. Liliental-Weber, J. Washburn, K. J. Duxstad, E. E. Haller, Z. Fan, S. N. Mohammad, W. Kim, A. E. Botchkarev, and H. Morko, "Microstructure of $\mathrm{Ti} / \mathrm{Al}$ and $\mathrm{Ti} / \mathrm{Al} / \mathrm{Ni} / \mathrm{Au}$ ohmic contacts for n-GaN," Appleed Physıcs Letters, vol. 69, no. 11, pp. 1556-1558, 1996.

[39] A. Motayed, R. Bathe, M. C. Wood, O. S. Diouf, R. D. Vispute, and S. Noor Mohammad, "Electrical, thermal, and microstructural characteristics of $\mathrm{Ti} / \mathrm{Al} / \mathrm{Ti} / \mathrm{Au}$ multilayer ohmic contacts to n-type GaN," Journal of Applzed Physıcs, vol. 93, no. 2, pp. 1087-1094, 2003.

[40] B. Van Daele, G. Van Tendeloo, W. Ruythooren, J. Derluyn, M. R. Leys, and M. Germain, "The role of al on ohmic contact formation on $\mathrm{n}$-type gan and algangan," Applied Physıcs Letters, vol. 87, no. 6, 2005. 
[41] B. P. Luther, S. E. Mohney, T. N. Jackson, M. Asif Khan, Q. Chen, and J. W. Yang, "Investigation of the mechanism for ohmic contact formation in al and ti/al contacts to n-type gan," Applied Physics Letters, vol. 70, no. 1, pp. 57-59, 1997.

[42] J. A. Bardwell, G. I. Sproule, Y. Liu, H. Tang, J. B. Webb, J. Fraser, and P. Marshall, "Comparison of two different $\mathrm{Ti} / \mathrm{Al} / \mathrm{Ti} / \mathrm{Au}$ ohmic metallization schemes for AlGaN/GaN," Journal of Vacuum Science and Technology B: Microelectronics and Nanometer Structures, vol. 20, no. 4, pp. 1444-1447, 2002.

[43] N. Miura, T. Oishi, T. Nanjo, M. Suita, Y. Abe, T. Ozeki, H. Ishikawa, and T. Egawa, "Effects of interfacial thin metal layer for high-performance Pt-Aubased Schottky contacts to AlGaN-GaN," IEEE Transactions on Electron Devices, vol. 51, no. 3, pp. 297-303, 2004.

[44] N. Miura, T. Nanjo, M. Suita, T. Oishi, Y. Abe, T. Ozeki, H. Ishikawa, T. Egawa, and T. Jimbo, "Thermal annealing effects on $\mathrm{Ni} / \mathrm{Au}$ based schottky contacts on $\mathrm{n}-\mathrm{GaN}$ and $\mathrm{AlGaN} / \mathrm{GaN}$ with insertion of high work function metal," Solid-State Electronics, vol. 48, no. 5, pp. 689-695, 2004.

[45] H. Hasegawa, T. Inagaki, S. Ootomo, and T. Hashizume, "Mechanisms of current collapse and gate leakage currents in AlGaN/GaN heterostructure field effect transistors," Journal of Vacuum Science and Technology B: Microelectronics and Nanometer Structures, vol. 21, no. 4, pp. 1844-1855, 2003.

[46] S. Haffouz, H. Tang, J. A. Bardwell, E. M. Hsu, J. B. Webb, and S. Rolfe, "AlGaN/GaN field effect transistors with C-doped GaN buffer layer as an electrical isolation template grown by molecular beam epitaxy," Solid-State Electronics, vol. 49 , no. 5 , pp. 802-807, 2005.

[47] F. Gonzlez-Posada, J. A. Bardwell, S. Moisa, S. Haffouz, H. Tang, A. F. Braa, and E. Muoz, "Surface cleaning and preparation in AlGaN/GaN-based HEMT processing as assessed by X-ray photoelectron spectroscopy," Applied Surface Science, vol. 253, no. 14, pp. 6185-6190, 2007.

[48] I. Cimalla, F. Will, K. Tonisch, M. Niebelschtz, V. Cimalla, V. Lebedev, G. Kittler, M. Himmerlich, S. Krischok, J. A. Schaefer, M. Gebinoga, A. Schober, T. Friedrich, and O. Ambacher, "AlGaN/GaN biosensor-effect of device processing steps on the surface properties and biocompatibility," Sensors and Actuators, B: Chemical, vol. 123, no. 2, pp. 740-748, 2007. 
[49] K. J. Kim, J. S. Jang, D. W. Moon, and H. J. Kang, "A method to determine the interface position and layer thickness in SIMS depth profiling of multilayer films," Metrologia, vol. 47, no. 3, pp. 253-261, 2010.

[50] J. F. Moulder, W. F. Stickle, P. E. Sobol, and K. D. Bomben, Handbook of X-ray Photoelectron Spectroscopy. USA: Perkin-Elemer Corporation, 1992.

[51] Z. H. Lu, J. P. McCaffrey, B. Brar, G. D. Wilk, R. M. Wallace, L. C. Feldman, and S. P. Tay, " $\mathrm{SiO}_{2}$ film thickness metrology by x-ray photoelectron spectroscopy," Applied Physics Letters, vol. 71, pp. 2764-2766, 1997.

[52] H.-J. Butt, K. Graf, and M. Kappl, Physics and chemistry of interfaces. USA: Wiley-VCH, 2003.

[53] C. Buchheim, V. Lebedev, V. Yanev, G. Kittler, M. Fischer, G. Ecke, O. Ambacher, V. Cimalla, S. Krischok, and J. A. Schaefer, "Surface modifications of AlGaN/GaN sensors for water base nano- and picodroplets," vol. 2, pp. 10071010, 2004.

[54] R. G. Wilson, S. J. Pearton, C. R. Abernathy, and J. M. Zavada, "Thermal stability of implanted dopants in GaN," Applied Physics Letters, p. 2238, 1995.

[55] C. Nguyen, P. Shah, E. Leong, M. Derenge, and K. Jones, "Si implant-assisted ohmic contacts to GaN," Solid-State Electronics, vol. 54, no. 10, pp. 1227-1231, 2010 .

[56] J. Burm, K. Chu, W. A. Davis, W. J. Schaff, L. F. Eastman, and T. J. Eustis, "Ultra-low resistive ohmic contacts on n-Gan using Si implantation," Applied Physics Letters, vol. 70, no. 4, pp. 464-466, 1997.

[57] F. Recht, L. McCarthy, S. Rajan, A. Chakraborty, C. Poblenz, A. Corrion, J. S. Speck, and U. K. Mishra, "Nonalloyed ohmic contacts in AlGaN/GaN HEMTs by ion implantation with reduced activation annealing temperature," IEEE Electron Device Letters, vol. 27, no. 4, pp. 205-207, 2006.

[58] S. J. Pearton, H. Cho, J. R. LaRoche, F. Ren, R. G. Wilson, and J. W. Lee, "Oxygen diffusion into $\mathrm{SiO}_{2}$-capped $\mathrm{GaN}$ during annealing," Applied Physics Letters, vol. 75, no. 19, pp. 2939-2941, 1999.

[59] C. F. Lin, H. C. Cheng, G. C. Chi, C. J. Bu, and M. S. Feng, "Improved contact performance of GaN film using si diffusion," Applied Physics Letters, vol. 76, no. 14 , pp. $1878-1880,2000$. 
[60] H. Ishida, Y. Hirose, T. Murata, Y. Ikeda, T. Matsuno, K. Inoue, Y. Uemoto, T. Tanaka, T. Egawa, and D. Ueda, "A high-power RF switch IC using AlGaN/GaN HFETs with single-stage configuration," IEEE Transactions on Electron Devices, vol. 52, no. 8, pp. 1893-1899, 2005.

[61] T. Murata, M. Hikita, H. Ishida, Y. Hirose, K. Inoue, Y. Uemoto, T. Tanaka, and D. Ueda, "Reduction of ohmic contact resistance of AlGaN/GaN HFETs by doping of thermally diffused $\mathrm{Si}$," International Meeting for Future of Electron Devices, p. 47, 2004.

[62] C. J. Pan, G. C. Chi, B. J. Pong, J. K. Sheu, and J. Y. Chen, "Si diffusion in p-GaN," Journal of Vacuum Science and Technology B: Microelectronics and Nanometer Structures, vol. 22, no. 4, pp. 1727-1730, 2004.

[63] S. Jang, F. Ren, S. J. Pearton, B. P. Gila, M. Hlad, C. R. Abernathy, H. Yang, C. J. Pan, J. . Chyi, P. Bove, H. Lahreche, and J. Thuret, "Si-diffused GaN for enhancement-mode GaN MOSFET on Si applications," Journal of Electronic Materials, vol. 35, no. 4, pp. 685-690, 2006.

[64] V. Adivarahan, M. Gaevski, A. Koudymov, J. Yang, G. Simin, and M. Khan, "Selectively doped high-power AlGaN/InGaN/GaN MOS-DHFET," Electron Device Letters, IEEE, vol. 28, no. 3, pp. $192-194,2007$.

[65] Y. Deng, V. Adivarahan, and A. Khan, "Selective doping and optimization of InGaN channel and InGaN back-barrier in deep submicron GaN heterojunction field effect transistor with a recessed gate," 2007.

[66] D. Kim and H. K. Baik, "Current conduction mechanism of Si/Ti-based ohmic contacts to n-GaN," Applied Physics Letters, vol. 77, no. 7, pp. 1011-1013, 2000.

[67] V. Desmaris, J. . Shiu, C. . Lu, N. Rorsman, H. Zirath, and E. . Chang, "Transmission electron microscopy assessment of the $\mathrm{Si}$ enhancement of $\mathrm{Ti} / \mathrm{Al} / \mathrm{Ni} / \mathrm{Au}$ Ohmic contacts to undoped AlGaN/GaN heterostructures," Journal of Applied Physics, vol. 100, no. 3, 2006.

[68] F. M. Mohammed, L. Wang, and I. Adesida, "Ultralow resistance Si-containing $\mathrm{Ti} / \mathrm{Al} / \mathrm{Mo} / \mathrm{Au}$ Ohmic contacts with large processing window for AlGaNGaN heterostructures," Applied Physics Letters, vol. 88, no. 21, 2006.

[69] N. Onojima, N. Hirose, T. Mimura, and T. Matsui, "Effects of Si deposition on AIGaN barrier surfaces in GaN heterostructure field-effect transistors," Applied Physics Express, vol. 1, no. 7, pp. 0711011-0711013, 2008. 
[70] N. Onojima, N. Hirose, T. Mimura, and T. Matsui, "Ultrathin AlN/GaN heterostructure field-effect transistors with deposition of Si atoms on AlN barrier surface," Applied Physics Letters, vol. 93, no. 22, 2008.

[71] S. Arulkumaran, T. Egawa, H. Ishikawa, T. Jimbo, and Y. Sano, "Surface passivation effects on $\mathrm{AlGaN} / \mathrm{GaN}$ high-electron-mobility transistors with $\mathrm{SiO}_{2}$, $\mathrm{Si}_{3} \mathrm{~N}_{4}$, and silicon oxynitride," Applied Physıcs Letters, vol. 84, no. 4, pp. 613$615,2004$.

[72] P. H. Fang, P. Bai, J. H. Kinnier, Z. Huan, and C. C. Schubert, "Temperature dependent formation of microcrystal and amorphous silicon by vacuum evaporation," Journal of Non-Crystallıne Solıds, vol. 59-60, no. PART 2, pp. 819-821, 1983.

[73] M. Steinert, J. Acker, S. Oswald, and K. Wetzig, "Study on the mechanism of silicon etching in $\mathrm{HNO}_{3}$-rich $\mathrm{HF} / \mathrm{HNO}_{3}$ mixtures," Journal of Physıcal Chemıstry $C$, vol. 111, no. 5, pp. 2133-2140, 2007.

[74] H. R. Philipp and E. A. Taft, "An optical characterization of native oxides and thin thermal oxides on silicon," Journal of Applzed Physzcs, vol. 53, no. 7, pp. 5224-5229, 1982.

[75] C. R. Werrett, A. K. Bhattacharya, and D. R. Pyke, "The validity of c1s charge referencing in the xps of oxidised al-si alloys," Applıed Surface Scıence, vol. 103, no. 4, pp. 403-407, 1996.

[76] N. Koshizaki, H. Umehara, and T. Oyama, "XPS characterization and optical properties of $\mathrm{Si} / \mathrm{SiO} 2, \mathrm{Si} / \mathrm{Al} 2 \mathrm{O} 3$ and $\mathrm{Si} / \mathrm{MgO}$ co-sputtered films," Thin Solıd Fllms, vol. 325, no. 1-2, pp. $130-136,1998$.

[77] M. Matsuoka, S. Isotani, W. Sucasaire, L. Zambom, and K. Ogata, "Chemical bonding and composition of silicon nitride films prepared by inductively coupled plasma chemical vapor deposition," Surface and Coatings Technology, vol. 204, no. $18-19$, pp. $2923-2927,2010$.

[78] D. Briggs and M. Seah, Practıcal Surface Analysis. Volume 1. Auger and X-ray Photoelectron Spectroscopy. Chichester, UK: John Wiley and Sons, 1990.

[79] J. Liang, L. Topor, A. Navrotsky, and M. Mitomo, "Silicon nitride: enthalpy of formation of the $\alpha$ - and $\beta$-polymorphs and the effect of $\mathrm{C}$ and $\mathrm{O}$ impurities," Journal of Materials Research, vol. 14, no. 5, pp. 1959-1968, 1999. 


\section{Appendix A}

\section{GaN IC FABRICATION PROCESS FLOW}

List of process flow specific abbreviations:

$\begin{array}{ll}\text { BOE } & \text { Buffered Oxide Etchant } \\ \text { DI } & \text { Deionized (Water) } \\ \text { HMDS } & \text { Hexamethyldisilazane, Adhesion Promoter } \\ \text { ICP } & \text { Inductively Coupled Plasma } \\ \text { IPA } & \text { Isopropyl Alcohol } \\ \text { RIE } & \text { Reactive Ion Etch }\end{array}$

\section{A.1 NRC's standard GaN IC Fabrication Process Flow (some information is intentionally omit- ted)}

1. Ohmic Contact Lithography

- Native oxide removal

- Double resist layer coat/bake

- UV exposure: GANFET Ohmic mask

- Develop. Inspect

2. Ohmic Metallization

- Microwave descum

- Pre clean 
- E-beam deposition: $\mathrm{Ti} / \mathrm{Al} / \mathrm{Ti} / \mathrm{Au}$ ohmic metal stack

- Lift-off

- Rinse: acetone, IPA, DI rinse

- Microwave descum

\section{Ohmic Metal Anneal}

- Inspect for compete lift-off prior to anneal

- RTA Anneal: 845C, 1 min. in N2 flow

- Measure: contact resistance and saturation currents

\section{Mesa Lithography}

- Photoresist coat/bake

- UV exposure: GANFET Mesa mask

- Develop. Inspect

\section{Mesa Etch in ICP}

- ICP etch

- Resist strip

- Clean: IPA, DI rinse

- Microwave descum/oxide removal

- Characterization: surface profiling with Dektak

\section{Gate Metal Optical Lithography}

- Native oxide removal

- Double resist layer coat/bake

- UV exposure: GANFET Gate Metal mask

- Develop. Inspect

\section{Gate Metallization}

- Microwave descum

- Pre clean

- Deposition: Pt based Schottky metal stack

- Lift-off

- Rinse: acetone, IPA, DI rinse

- Microwave descum

8. Gate Metal Anneal

- Inspect for complete lift-off prior to anneal

- RTA Anneal in N2 flow

\section{Silicon Nitride Passivation}

- Microwave descum 
- Pre-clean

- Deposit SiN layer

10. Pattern for RIE Etch (VIA1)

- Resist coat/bake

- UV exposure: GANFET VIA1 mask

- Develop, DI rinse

- Microwave descum. Inspect

11. Silicon Nitride VIA1 Etch

- ICP etch

- Resist strip

- Rinse: IPA, DI rinse

- Microwave descum. Inspect

12. Pattern for $\mathbf{1 M E}$

- Thermoplastic coat/bake

- Resist coat/bake

- UV exposure: GANFET 1ME Mask

- Resist develop, DI rinse

- Microwave descum

- DUV exposure

- Thermoplastic develop. Inspect

13. 1ME Metallization

- Microwave descum

- Deposition: Au based 1ME metal stack

- Lift-off

- Rinse: acetone/IPA

- RTA anneal in N2 flow. Inspect

\section{A.2 Batch-1 Fabrication Process Flow}

NOTE: '*' denotes a standard NRC's process step.

1. a-Si Thin Film Coating

- Pre clean

- E-beam evaporation: $30 \mathrm{~nm}$ of a-Si on designated samples. Use magnets to protect corners for probing access. Include Si test wafer

\section{SiON Thin Film Coating}

- Pre clean 
- PECVD: $60 \mathrm{~nm}$ of silicon oxinitride (SiON) on designated samples. Include Si test wafer

3. Surface Encapsulation with $\mathrm{SiO}_{2}$

- PECVD: $100 \mathrm{~nm}$ of $\mathrm{SiO}_{2}$ on thin film coated samples. Include Si test wafer

\section{Corner Patterning and Wet Etching for Probing Access}

- Clean: Acetone, IPA, DI rinse

- Photoresist coat/bake

- Expose corners manually with microscope light

- Resist develop, DI rinse.

- $\mathrm{BOE}$ etch test wafers: determine $\mathrm{SiO}_{2}$ and $\mathrm{SiO}_{2}+\mathrm{SiON}$ etch rates by observing hydrophobic properties of the $\mathrm{Si}$ wafers

- BOE etch: etch $\mathrm{SiO}_{2}$ and $\mathrm{SiO}_{2}+\mathrm{SiON}$ using measured times. DI rinse

- Resist strip: acetone, IPA, DI rinse

5. Stepwise Heat Treatment Anneals and Hall Measurements

- Initial electrical Hall measurement using access corners

- Clean: acetone, IPA, DI rinse

- RTA Anneal: $T_{1}=400 \mathrm{C}, t_{1}$ min. in N2 flow

- Electrical Hall measurement using access corners

- Selectively repeat the anneals up to $T_{n}=950 \mathrm{C}$

6. Wet Etch of $\mathrm{SiO}_{2}$, a-Si and SiON Films

- Clean: Acetone, IPA, DI rinse

- Use previously determined wet etch duration for $\mathrm{SiO}_{2}$ and $\mathrm{SiON}$ wet etch in BOE. DI rinse. Inspect

- a-Si wet etch in 1:3 HF:HNO 3,5 sec. DI rinse. Inspect

- Electrical Hall measurement

7. *Ohmic Contact Lithography

8. *Ohmic Metallization

9. *Ohmic Metal Anneal

10. Post Ohmic Anneal Electrical Measurements

- C-TLM ohmic contact and sheet resistance measurements

11. *Mesa Lithography

12. *Mesa Etch in ICP

13. *Gate Metal Optical Lithography

14. *Gate Metallization 
15. *Gate Metal Anneal

16. Electrical Measurements, Unpassivated Devices

- C-TLM ohmic contact and sheet resistance

- TLM ohmic contact and sheet resistance

- HEMT DC and pulsed I-V/ Current collapse

- HEMT gate Schottky junction I-V

- HEMT drain-source breakdown voltage

17. *Silicon Nitride Passivation

18. *Pattern for RIE Etch (VIA1)

19. *Silicon Nitride VIA1 Etch

20. Electrical Measurements, Passivated Devices

- C-TLM ohmic contact and sheet resistance

- TLM ohmic contact and sheet resistance

- HEMT DC and pulsed I-V/ Current collapse

- HEMT gate Schottky junction I-V

- HEMT drain-source breakdown voltage

21. *Pattern for $1 \mathrm{ME}$

22. 1ME Metallization

- Microwave descum

- Deposition: Au based 1ME metal stack

- Lift-off

- Rinse: acetone/IPA

23. Post 1ME Electrical Measurements

- C-TLM ohmic contact and sheet resistance

- TLM ohmic contact and sheet resistance

\section{A.3 Batch-2 Fabrication Process Flow}

NOTE: '*' denotes a standard NRC's process step.

1. a-Si $(30 \mathrm{~nm})$ Thin Film Coating

- Pre clean

- E-beam evaporation: $30 \mathrm{~nm}$ of a-Si on designated samples. Include Si test wafer

2. a-Si (5 nm) Thin Film Coating

- Pre clean

- E-beam evaporation: $5 \mathrm{~nm}$ of a-Si on designated samples at $1 \AA / \mathrm{sec}$ dep. rate. Include Si test wafer 


\section{Surface Encapsulation with $\mathrm{SiO}_{2}$}

- PECVD: $100 \mathrm{~nm}$ of $\mathrm{SiO}_{2}$ on $30 \mathrm{~nm}$ a-Si thin film coated samples. Include Si test wafer

4. Heat Treatment Anneals and Hall Measurements

- RTA Anneal: designated $30 \mathrm{~nm}$ a-Si coated samples, 850C, $15 \mathrm{~min}$. in N2 flow

- RTA Anneal: designated $30 \mathrm{~nm}$ a-Si coated samples, 950C, 5 min. in N2 flow

- RTA Anneal: designated $30 \mathrm{~nm}$ a-Si coated samples, 1,000C, 5 min. in N2 flow

5. Wet Etch of $\mathrm{SiO}_{2}$ and a-Si Films

- Clean: Acetone, IPA, DI rinse

- $\mathrm{BOE}$ etch test wafers: determine $\mathrm{SiO}_{2}$ etch rates by observing hydrophobic properties of the Si wafers

- Use previously determined wet etch duration for $\mathrm{SiO}_{2}$ wet etch in $\mathrm{BOE}$. DI rinse. Inspect

- Perform Hall measurement on a new control sample. Add to $\mathrm{HF}: \mathrm{HNO}_{3}$ wet etch batch

- a-Si wet etch in 1:3 HF: $\mathrm{HNO}_{3}, 10$ sec. DI rinse. Inspect

- Electrical Hall measurement on a new control sample

6. *Ohmic Contact Lithography

7. *Ohmic Metallization

8. Stepwise Ohmic Metal Anneal

- Inspect for compete lift-off prior to anneal

- RTA Anneal: $T_{1}=750 \mathrm{C}, 1 \mathrm{~min}$. in N2 flow

- Measure: c-TLM contact and sheet resistance, saturation currents over $2 \mu \mathrm{m}$ c-TLM gap

- Repeat the anneals for increasing temperatures until the best ohmic contacts are achieved

\section{SIMS and XPS Measurements}

- Perform SIMS profiling on dedicated samples

- Perform XPS chemical composition measurement on dedicated samples

\section{A.4 Batch-3 Fabrication Process Flow}

NOTE: '*' denotes a standard NRC's process step.

1. a-Si (30 nm) Thin Film Coating

- Pre clean

- E-beam evaporation: $30 \mathrm{~nm}$ of a-Si on designated samples. Include Si test wafer

2. a-Si $(5 \mathrm{~nm})$ Thin Film Coating 
- Pre clean

- E-beam evaporation: $5 \mathrm{~nm}$ of a-Si on designated samples at $1 \AA / \mathrm{sec}$ dep. rate. Include Si test wafer

\section{Surface Encapsulation with $\mathrm{SiO}_{2}$}

- PECVD: $100 \mathrm{~nm}$ of $\mathrm{SiO}_{2}$ on $30 \mathrm{~nm}$ a-Si thin film coated samples. Include Si test wafer

\section{Heat Treatment Anneals and Hall Measurements}

- RTA Anneal: $30 \mathrm{~nm}$ a-Si coated samples, 950C, 5 min. in N2 flow

\section{Wet Etch of $\mathrm{SiO}_{2}$ and a-Si Films}

- Clean: Acetone, IPA, DI rinse

- BOE etch test wafers: determine $\mathrm{SiO}_{2}$ etch rates by observing hydrophobic properties of the Si wafers

- Use previously determined wet etch duration for $\mathrm{SiO}_{2}$ wet etch in $\mathrm{BOE}$. DI rinse. Inspect

- a-Si wet etch in 1:3 $\mathrm{HF}: \mathrm{HNO}_{3} 10 \mathrm{sec}$. DI rinse. Inspect

\section{XPS Measurements}

- Perform XPS chemical composition measurement on dedicated samples

\section{Surface Contact Angle Measurement}

- Measure contact angle

- Clean: Acetone, IPA, DI Rinse. Measure contact angle

- Clean: Acetone, scrub with IPA, DI rinse. Measure contact angle

- HMDS prime. Measure contact angle

- Photoresist coat/bake. Develop. DI rinse. Measure contact angle

- Microwave descum. Measure contact angle

- Standard ohmic met. dep. pre clean. DI rinse. Measure contact angle

- DI rinse. Measure contact angle

- Store samples for $\sim 24$ hours. Measure contact angle

\section{XPS Measurements}

- Perform XPS chemical composition measurement on dedicated samples

\section{9. *Ohmic Contact Lithography}

10. *Ohmic Metallization

\section{Stepwise Ohmic Metal Anneal}

- Inspect for compete lift-off prior to anneal

- RTA Anneal: $T_{1}=825 \mathrm{C}, 1 \mathrm{~min}$. in N2 flow

- Measure: c-TLM contact and sheet resistances, saturation current over $2 \mu \mathrm{m}$ c-TLM gap 
- Repeat the anneals for increasing temperatures until best ohmic contacts are achieved

12. *Mesa Lithography

13. *Mesa Etch in ICP

14. *Gate Metal Optical Lithography

15. *Gate Metallization

16. *Gate Metal Anneal

17. Electrical Measurements, Unpassivated Devices

- TLM ohmic contact and sheet resistance

- HEMT DC and pulsed I-V / Current collapse

- HEMT transconductance and threshold voltage

18. *Silicon Nitride Passivation

19. *Pattern for RIE Etch (VIA1)

20. *Silicon Nitride VIA1 Etch

21. *Pattern for $1 \mathrm{ME}$

22. *1ME Metallization

23. Electrical Measurements, Passivated Devices

- C-TLM/TLM ohmic contact and sheet resistance, current density over $2 \mu \mathrm{m}$ c-TLM gap

- HEMT DC and pulsed I-V / Current collapse

- HEMT transconductance and threshold voltage

- HEMT gate Schottky junction I-V

- HEMT gate leakage versus drain voltage for standard and field plate assisted devices 